WSRC-TR-2000-00218, Revision 1

\title{
AM/CM VITRIFICATION PROCESS: VITRIFICATION MATERIAL BALANCE CALCULATIONS (U)
}

F. G. Smith, III

Westinghouse Savannah River Company

Savannah River Site

Aiken, SC 29808

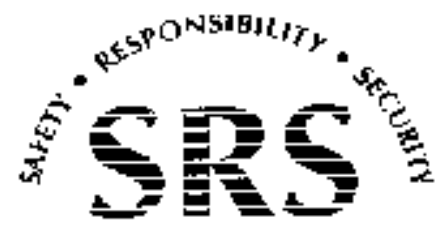

PREPARED FOR THE U.S. DEPARTMENT OF ENERGY UNDER CONTRACT NO. DE-AC09-96SR18500 
This document was prepared in conjunction with work accomplished under Contract No.

DE-AC09-96SR18500 with the U.S. Department of Energy.

\section{DISCLAIMER}

This report was prepared as an account of work sponsored by an agency of the United States Government. Neither the United States Government nor any agency thereof, nor any of their employees, makes any warranty, express or implied, or assumes any legal liability or responsibility for the accuracy, completeness, or usefulness of any information, apparatus, product or process disclosed, or represents that its use would not infringe privately owned rights. Reference herein to any specific commercial product, process or service by trade name, trademark, manufacturer, or otherwise does not necessarily constitute or imply its endorsement, recommendation, or favoring by the United States Government or any agency

thereof. The views and opinions of authors expressed herein do not necessarily state or reflect those of the United States Government or any agency thereof.

This report has been reproduced directly from the best available copy.

Available for sale to the public, in paper, from: U.S. Department of Commerce, National Technical Information Service, 5285 Port Royal Road, Springfield, VA 22161, phone: (800)

553-6847, fax: (703) 605-6900, email: orders@ntis.fedworld.gov online ordering: http://www.ntis.gov/ordering.htm

Available electronically at http://www.doe.gov/bridge

Available for a processing fee to U.S. Department of Energy and its contractors, in paper, from: U.S. Department of Energy, Office of Scientific and Technical Information, P.O. Box 62, Oak Ridge, TN 37831-0062, phone: (865 ) 576-8401, fax: (865) 576-5728, email: reports@ adonis.osti.gov 
WSRC-TR-2000-00218, Revision 1

Keywords: AM/CM, Material Balance, Vitrification

Retention: Permanent

\section{AM/CM VITRIFICATION PROCESS: VITRIFICATION MATERIAL BALANCE CALCULATIONS (U)}

F. G. Smith, III

Publication Date: December 12, 2000

Westinghouse Savannah River Company

Savannah River Site

Aiken, SC 29808

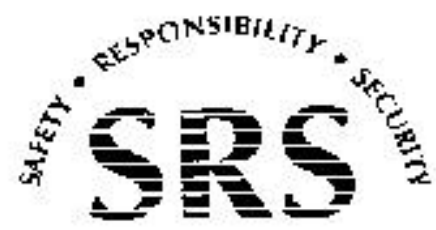

PREPARED FOR THE U.S. DEPARTMENT OF ENERGY UNDER CONTRACT NO. DE-AC09-96SR18500 


\section{APPROVALS}

F. G. Smith, III, Author Immobilization Technology Section

D. C. Witt, Technical Reviewer Immobilization Technology Section

L. F. Landon, Manager

Immobilization Technology Section
Date

Date

Date 


\section{TABLE OF CONTENTS}

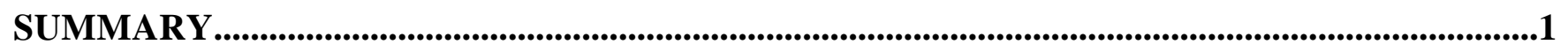

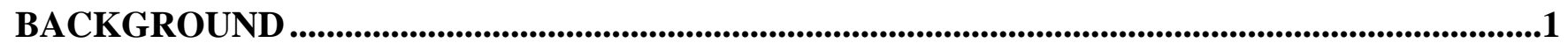

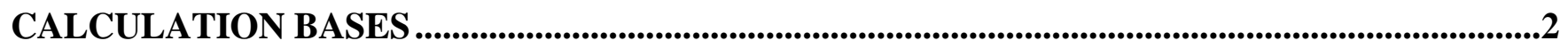

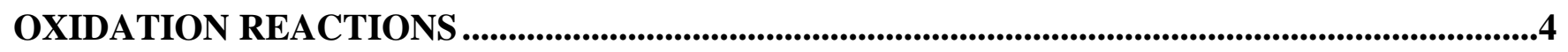

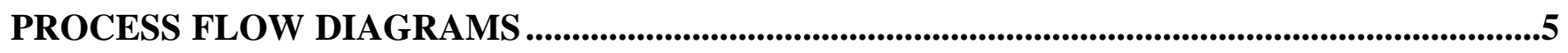

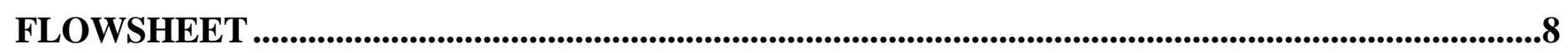

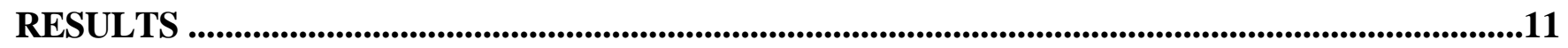

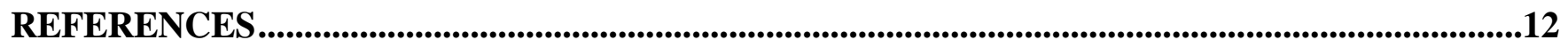

APPENDIX A..............................................................................................................................................13

APPENDIX B ................................................................................................................................................28 


\section{SUMMARY}

This report documents material balance calculations for the Americium/Curium vitrification process and describes the basis used to make the calculations. The material balance calculations reported here start with the solution produced by the Am/Cm pretreatment process as described separately in Reference [1]. Following pretreatment, small batches of the product will be further treated with an additional oxalic acid precipitation and washing. The precipitate from each batch will then be charged to the Am/Cm melter with glass cullet and vitrified to produce the final product. The material balance calculations in this report are designed to provide projected compositions of the melter glass and off-gas streams. Except for decanted supernate collected from precipitation and precipitate washing, the flowsheet neglects side streams such as acid washes of empty tanks that would go directly to waste. Complete listings of the results of the material balance calculations are provided in the Appendices to this report.

This revision is required to reflect minor changes resulting from a revision to the pretreatment material balance described in Reference [1], which changed some of the values in the vitrification calculations. No changes have been made to any of the bases for the vitrification material balance.

\section{BACKGROUND}

Based on 1998 analytical measurements, Tank 17.1 contains approximately 11,000 liters of Americium/Curium solution at about $8 \mathrm{M}$ nitric acid. The material stored in Tank 17.1 will be diluted, denitrated using formic acid, and precipitated with oxalic acid to separate actinides and lanthanides from transition metals, aluminum and alkali constituents. The precipitate will then be washed to further remove impurities. Following these clean-up steps, the solution will be treated with concentrated nitric acid to redissolve the precipitate and destroy oxalate. The solution will then be water stripped, denitrated to reduce the nitric acid concentration, and concentrated to produce a solution having about $100 \mathrm{~g} / \mathrm{l}$ of solids on an oxide basis in $1.0 \mathrm{M}$ nitric acid. These processing steps are included in the pretreatment material balance [1].

Approximately seven liter batches of the pretreated material will then be precipitated and washed with oxalic acid to further remove transition metals and convert the actinides and lanthanides to oxalate salts. This solution will be fed to the melter along with a glass cullet and heated to remove water and form the Am/Cm glass product. During melter heating, the water in solution is driven off, oxalate ions are burned to carbon dioxide and carbon monoxide and nitrate ions form $\mathrm{NO}_{\mathrm{x}}$ gas species. The material balance was implemented in an Excel $^{\mathrm{TM}}$ spreadsheet that relies on experimental data, empirical formulas and simplifying assumptions to make the flowsheet calculations. 


\section{CALCULATION BASES}

The calculations in this report are designed to determine the compositions of the melter feed, the glass product from the Americium/Curium vitrification campaign, and the melter off-gas streams. The material balance starts with the product of the pretreatment process. These material balance calculations used the most recent (1998) analytical measurements of the composition of the solution in Tank 17.1 as a starting point. Table 1 summarizes some of the basic assumptions used to make the vitrification material balance calculations.

Table 1. Calculation basis for vitrification material balance.

\begin{tabular}{|cc|}
\hline Parameter & Assumed Value \\
\hline Glass cullet & $25 \mathrm{Sr}$ ABS-F \\
Waste loading in glass as oxides & $32 \%$ \\
Lanthanide loading in glass as oxides & $49 \%$ \\
Melter feed batch & 6.91 liters \\
Melter off-gas pressure & -2 inches water \\
Melter off-gas temperature & $200-700{ }^{\circ} \mathrm{C}$ \\
Solids entrainment in off-gas & $0.1 \%$ of melter feed \\
Cesium volatility & $20 \%$ \\
\hline
\end{tabular}

The material balance calculations also relied on the following bases and assumptions:

- Salts of the metal impurities remaining in Tank 17.1 are completely soluble. The true chemical form of the metal impurity salts is uncertain. Choi [2] has determined a composition based on the chemical analysis of Tank 17.1 that best conserves mass and satisfies electroneutrality. This proposed composition was used for the pretreatment flowsheet. During the redissolving step, where concentrated nitric acid is added to the solution, the metal impurities are likely converted to the nitrate salts. However, to simplify the calculations, it is assumed that the chemical species proposed by Choi remain intact throughout the entire pretreatment process. During vitrification, these materials are converted into the oxide form.

- Following the assumption used by Rudisill [3], trace amounts of the elements Tb, Dy, Ho, Er, Tm, $\mathrm{Yb}$ and $\mathrm{Lu}$ are assumed to be present in the Tank 17.1 solution. The concentrations that are assumed for these elements represent the minimum detectable quantities that could be present in the Tank 17.1 solution.

- Solubilities of oxalate salts of the lanthanides, uranium and transuranic metals are based on experimental data. The data obtained by Beck [4] and Rudisill [3] were used to calculate the solubility of the oxalate salts of Lanthanide metals. Full details of the solubility calculation are provided in the report on the pretreatment material balance [1]. 
- The oxalate precipitate layer has an apparent specific volume of $0.5 \mathrm{l} / \mathrm{mole}$ [4].

- When streams are mixed, if the final volume is not specified, it is simply assumed that the mixing volumes are additive. The mixture density is then calculated from the ratio of solution mass to volume.

- Air purges to the tanks are calculated using the methodology developed by Marek [5] to limit the hydrogen concentration in the vapor space of these vessels to $25 \%$ of the Lower Flammability Limit. It is assumed that the air purge is saturated air at $20^{\circ} \mathrm{C}$. The solution volumes shown in the Table 2 were used as a basis for the air purge calculations. These are the volumes of solution in the tanks when the greatest air purge is required.

Table 2. Vessel Volumes for Air Purge Calculations

\begin{tabular}{|c|c|}
\hline Vessel & Solution Volume (liters) \\
\hline Liquid Feed Tank & 48.0 \\
\hline Batch Control Tank & 6.91 \\
\hline Precipitator & 6.91 \\
\hline
\end{tabular}

- The air sweep over the melter is assumed to be saturated air at $20^{\circ} \mathrm{C}$. Even though saturated air at a higher temperature will carry more water vapor, the worst case with the greatest dilution air or offgas heating requirement was found to be colder air. Most of the off-gas mass comes directly from the air sweep and not from the melter. Therefore, colder air which is more easily condensed is the limiting operating condition that requires the greatest dilution air or off-gas heating. The off-gas heating or dilution airflow is set to give a relative humidity of $70 \%$ in the mixed off-gas $[6,7]$.

- $0.10 \%$ of the feed material added to the melter is lost to entrainment in the off-gas.

- $20.0 \%$ of the cesium added to the melter is volatilized into the off-gas during the drying stage.

- $20.0 \%$ of the carbon monoxide in the melter off-gas from the calcination reactions is burned to carbon dioxide.

- $50.0 \%$ of the $\mathrm{NO}_{\mathrm{x}}$ in the melter off-gas is decomposed to nitrogen and oxygen.

- Off-gases from melter drying, calcination and vitrification operations are included in the combined melter off-gas streams. Gas releases from the melter are converted from absolute amounts into flow rates by assuming a one-hour duration for each of the melter steps. The one hour duration assumed for each of the melter steps is likely low and therefore conservative for calculating off-gas flows.

- The material loading in the glass product is calculated to give $49.0 \%$ actinide and lanthanide oxides.

- Based on simulant experiments performed at TNX, the melter feed batch size is 6.91 liters. 


\section{OXIDATION REACTIONS}

The following oxidation reactions are applied to convert the salts in the melter feed solution into metal oxides and gas phase products. In some cases, the reaction can be applied to more than the one element used to illustrate the stoichiometry. In these cases, the metals that the reaction applies to are listed in parentheses to the right of the example reaction equation.

Oxalate salts:

$$
\begin{aligned}
& \mathrm{La}_{2}\left(\mathrm{C}_{2} \mathrm{O}_{4}\right)_{3} \rightarrow \mathrm{La}_{2} \mathrm{O}_{3}+3 \mathrm{CO}+3 \mathrm{CO}_{2} \quad \text { (Lanthanides, Am, Cm) } \\
& \mathrm{Np}_{2} \mathrm{O}_{4}\left(\mathrm{C}_{2} \mathrm{O}_{4}\right) \rightarrow \mathrm{Np}_{2} \mathrm{O}_{5}+\mathrm{CO}+\mathrm{CO}_{2} \\
& \mathrm{Pu}\left(\mathrm{C}_{2} \mathrm{O}_{4}\right)_{2} \rightarrow \mathrm{PuO}_{2}+2 \mathrm{CO}+2 \mathrm{CO}_{2} \\
& \mathrm{UO}_{2}\left(\mathrm{C}_{2} \mathrm{O}_{4}\right) \rightarrow \mathrm{UO}_{3}+\mathrm{CO}+\mathrm{CO}_{2} \\
& \mathrm{Fe}_{2}\left(\mathrm{C}_{2} \mathrm{O}_{4}\right)_{3} \rightarrow \mathrm{Fe}_{2} \mathrm{O}_{3}+3 \mathrm{CO}_{2}+3 \mathrm{CO} \\
& \mathrm{H}_{2}\left(\mathrm{C}_{2} \mathrm{O}_{4}\right) \rightarrow \mathrm{H}_{2} \mathrm{O}+\mathrm{CO}_{2}+\mathrm{CO}
\end{aligned}
$$

Nitrate salts:

$$
\begin{array}{ll}
2 \mathrm{Al}\left(\mathrm{NO}_{3}\right)_{3} \rightarrow \mathrm{Al}_{2} \mathrm{O}_{3}+6 \mathrm{NO}_{\mathrm{x}}+3 \mathrm{O}_{2} & (\mathrm{Al}, \mathrm{Fe}, \mathrm{Cr}) \\
\mathrm{Ca}\left(\mathrm{NO}_{3}\right)_{2} \rightarrow \mathrm{CaO}+2 \mathrm{NO}_{\mathrm{x}}+\mathrm{O}_{2} & (\mathrm{Ca}, \mathrm{Ni}, \mathrm{Mn}, \mathrm{Zn}) \\
\mathrm{Zr}\left(\mathrm{NO}_{3}\right)_{4} \rightarrow \mathrm{ZrO}_{2}+4 \mathrm{NO}_{\mathrm{x}}+2 \mathrm{O}_{2} & \\
2 \mathrm{H}\left(\mathrm{NO}_{3}\right) \rightarrow \mathrm{H}_{2} \mathrm{O}+2 \mathrm{NO}_{\mathrm{x}}+\mathrm{O}_{2} & (\mathrm{H}, \mathrm{Na}, \mathrm{K})
\end{array}
$$

$\underline{\text { Nitrite salts: }}$

$$
2 \mathrm{Na}\left(\mathrm{NO}_{2}\right) \rightarrow \mathrm{Na}_{2} \mathrm{O}+2 \mathrm{NO}_{\mathrm{x}}
$$

Sulfate salts:

$$
4 \mathrm{Al}_{2}\left(\mathrm{SO}_{4}\right)_{3} \rightarrow 4 \mathrm{Al}_{2} \mathrm{O}_{3}+12 \mathrm{SO}_{\mathrm{x}}+3 \mathrm{O}_{2} \quad(\mathrm{Al}, \mathrm{Fe})
$$

Phosphate salts:

$$
2 \mathrm{Fe}\left(\mathrm{PO}_{4}\right) \rightarrow \mathrm{Fe}_{2} \mathrm{O}_{3}+2 \mathrm{PO}_{\mathrm{x}}
$$

Fluoride salts:

$$
2 \mathrm{AlF}_{3}+3 \mathrm{H}_{2} \mathrm{O} \rightarrow \mathrm{Al}_{2} \mathrm{O}_{3}+6 \mathrm{HF}
$$

Chloride salts:

$$
2 \mathrm{FeCl}_{3}+3 \mathrm{H}_{2} \mathrm{O} \rightarrow \mathrm{Fe}_{2} \mathrm{O}_{3}+6 \mathrm{HCl}
$$


Sodium salt reactions in the material balance that produce no off-gas are:

$$
\begin{aligned}
& \mathrm{Na}_{2}\left(\mathrm{SiO}_{3}\right) \rightarrow \mathrm{Na}_{2} \mathrm{O}+\mathrm{SiO}_{2} \\
& \mathrm{Na}_{2}\left(\mathrm{~B}_{4} \mathrm{O}_{7}\right) \rightarrow \mathrm{Na}_{2} \mathrm{O}+2 \mathrm{~B}_{2} \mathrm{O}_{3}
\end{aligned}
$$

Oxides of nitrogen, sulfur and phosphorous are assumed to be equal molar mixtures of true oxides. That is, we take the $\mathrm{x}$ oxides to be:

$$
\begin{aligned}
& \mathrm{NO}_{\mathrm{x}}=1 / 2\left(\mathrm{NO}+\mathrm{NO}_{2}\right) \\
& \mathrm{SO}_{\mathrm{x}}=1 / 2\left(\mathrm{SO}_{2}+\mathrm{SO}_{3}\right) \\
& \mathrm{PO}_{\mathrm{x}}=1 / 2\left(\mathrm{PO}_{2}+\mathrm{PO}_{3}\right)
\end{aligned}
$$

At the projected melter plenum temperatures, we assume that $20 \%$ of the carbon monoxide is oxidized to carbon dioxide according to the reaction:

$$
\mathrm{CO}+1 / 2 \mathrm{O}_{2} \rightarrow \mathrm{CO}_{2}
$$

At the melter gas temperatures, we also assume that $50 \%$ of the $\mathrm{NO}_{\mathrm{x}}$ is thermally decomposed to $\mathrm{N}_{2}$ and $\mathrm{O}_{2}$ according to the reaction

$$
2 \mathrm{NO}_{\mathrm{x}} \rightarrow \mathrm{N}_{2}+1 \frac{1 / 2}{2} \mathrm{O}_{2}
$$

Based on the reactions listed above, the gaseous components of the melter off-gas stream are:

$$
\mathrm{O}_{2}, \mathrm{~N}_{2}, \mathrm{H}_{2} \mathrm{O}, \mathrm{CO}, \mathrm{CO}_{2}, \mathrm{NO}_{\mathrm{x}}, \mathrm{SO}_{\mathrm{x}}, \mathrm{PO}_{\mathrm{x}}, \mathrm{HF} \text { and } \mathrm{HCl}
$$

Oxygen, nitrogen and some water vapor are added to the melter off-gas from airflow into the melter vapor space. Water is volatilized into the off-gas from the melter feed.

\section{PROCESS FLOW DIAGRAMS}

Figures 1 through 2 show process flow diagrams for the melter feed preparation and vitrification parts of the $\mathrm{Am} / \mathrm{Cm}$ vitrification process as modeled by the material balance calculations. Stream numbers shown in the figures correspond to the column numbers assigned in the material balance spreadsheet. All streams in the material balance calculations are identified on the figures. Page numbers given in the titles indicate the pages in the spreadsheet where the streams are listed. Both the stream and spreadsheet page numbers continue from the numbering in the pretreatment material balance spreadsheet. 
WSRC-TR-2000-00218, Revision 1

Page 6 of 42

Melter Feed Preparation (Spreadsheet Pages 17-22,25)

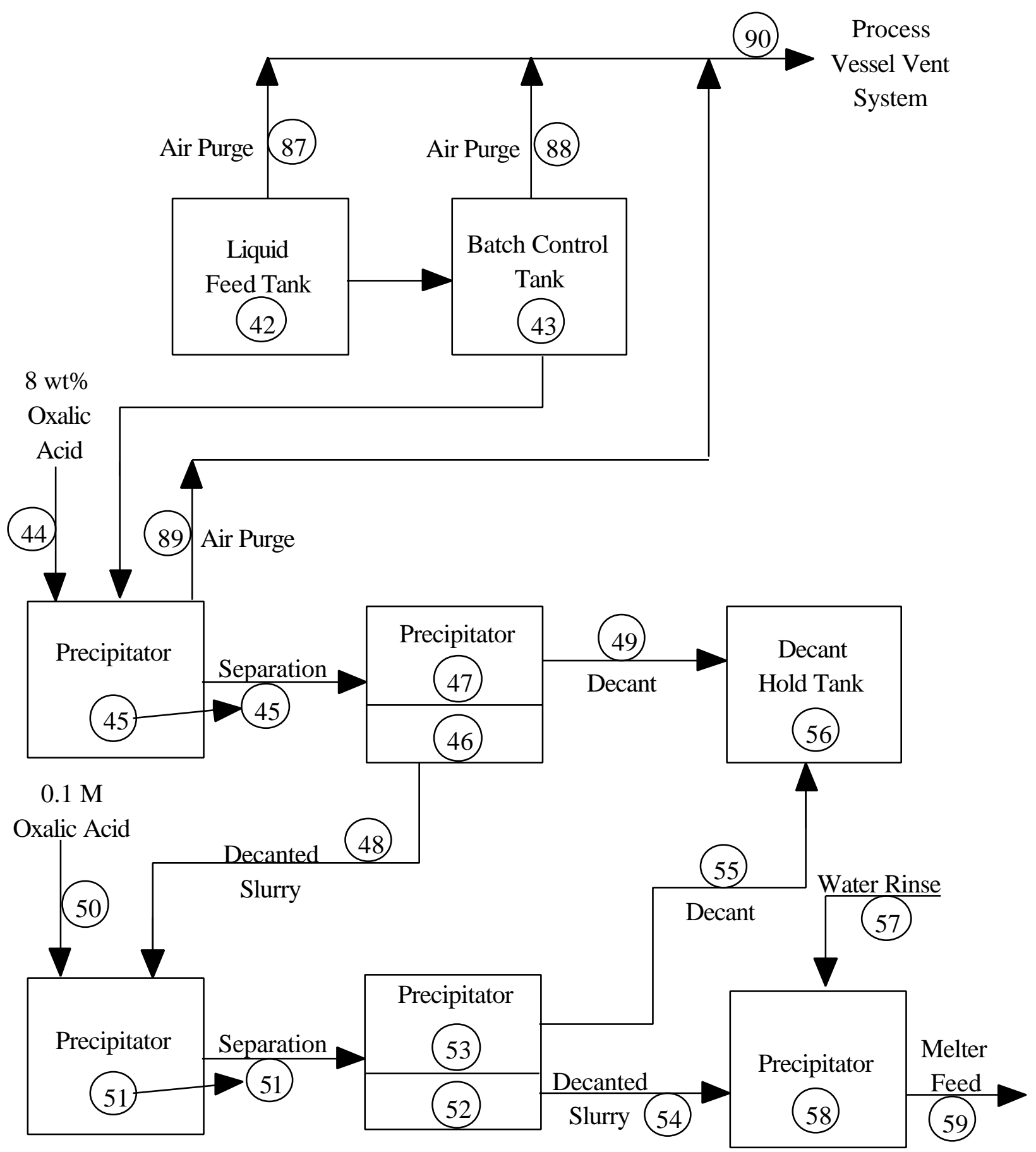

Figure 1. Flow diagram for $\mathrm{Am} / \mathrm{Cm}$ vitrification melter feed preparation process. 

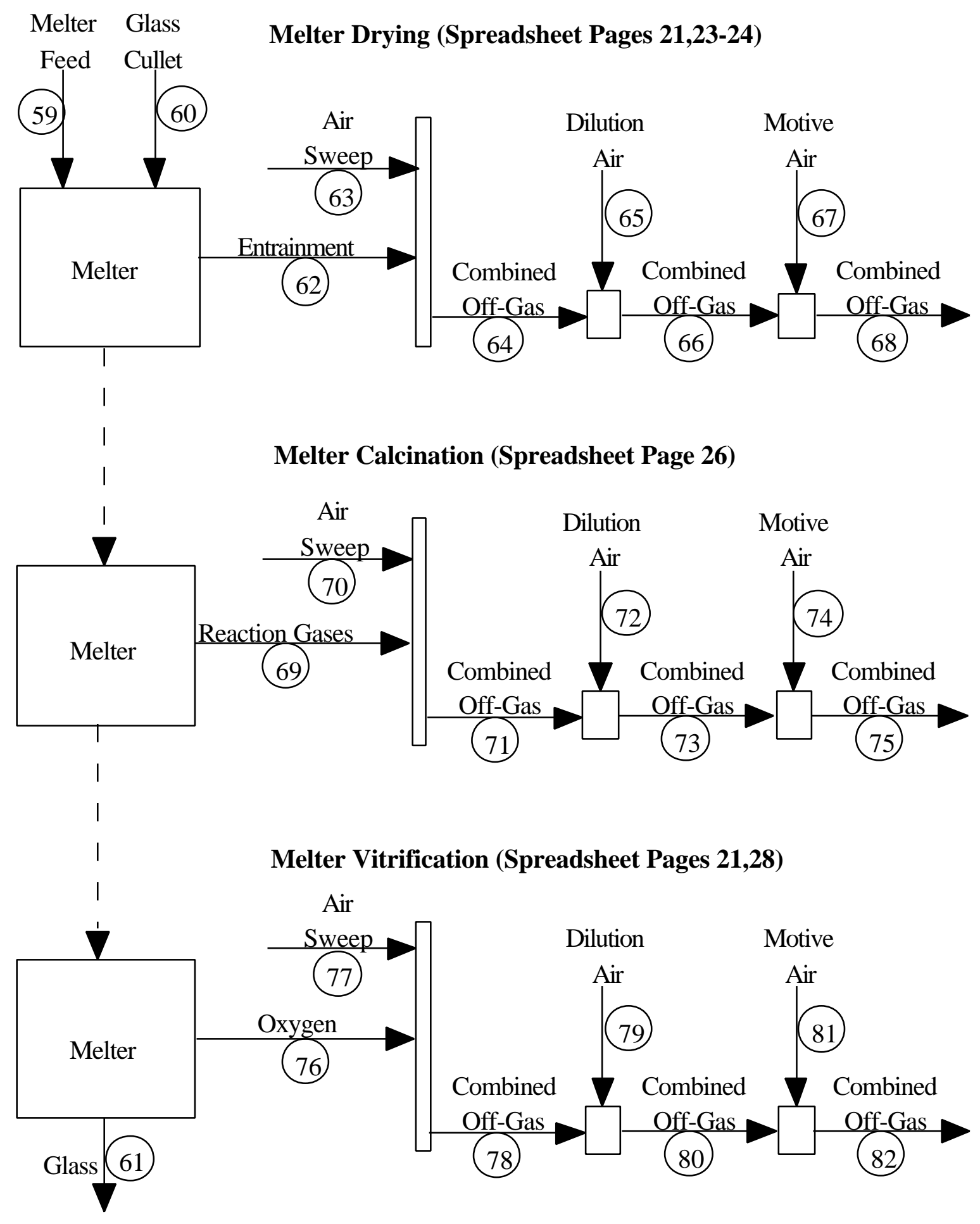

Figure 2. Flow diagram for Am/Cm vitrification and melter off-gas process. 


\section{FLOWSHEET}

The pretreatment material balance for processing steps prior to stream 43 has been reported separately [1]. Oxalic acid precipitation and washing steps that further treat the melter feed are included as part of the melter feed preparation process. These oxalic acid precipitation and washing calculations are based on the same solubility data used previously for the pre-treatment material balance. It was found that using a constant volume ratio of adding 1.74 liter of $8 \%$ oxalic acid to each liter of feed solution produced a product having very close to the desired $0.3 \mathrm{M}$ excess acid (see Stream 45). Therefore, this simplified calculation was used in the calculations. A listing of the streams in the vitrification material balance flowsheet and brief descriptions of each stream are provided in Tables 3 and 4 .

Calculations are made in the material balance to determine the composition of glass product from the $\mathrm{Am} / \mathrm{Cm}$ vitrification campaign and to estimate the average composition of the melter off-gas stream. Stream 60 gives the composition of the glass cullet (25 Sr ABS, [8]) in total grams and as the weight percent oxides. It is assumed that a stochiometric mixture of carbon monoxide and carbon dioxide is produced by the oxidation reactions in the melter. Subsequently, it is assumed that $20 \%$ of the carbon monoxide is burned to carbon dioxide in the off-gas. As assumed in the previous material balance calculations, $50 \%$ of the $\mathrm{NO}_{\mathrm{x}}$ in the off-gas is decomposed to $\mathrm{N}_{2}$ and $\mathrm{O}_{2}$. Also, as in previous material balances, the off-gas is converted from total mass into a mass flow rate by assuming representative times for the melter operating steps. Three stages of melter operation are considered:

1. Drying phase from $50{ }^{\circ} \mathrm{C}$ to $200{ }^{\circ} \mathrm{C}$ that removes water from the feed solution.

2. Calcination phase from $200{ }^{\circ} \mathrm{C}$ to $700{ }^{\circ} \mathrm{C}$ where conversion of the feed material into oxides takes place.

3. Vitrification phase from $700{ }^{\circ} \mathrm{C}$ to $1450{ }^{\circ} \mathrm{C}$ where $\mathrm{Ce}^{\mathrm{IV}}$ is reduced to $\mathrm{Ce}$ III .

The material balance assumes that the melter processes occur uniformly over the specified time. The off-gas flow is then calculated by computing the total gas released from the melter and dividing by the specified operating time. For example, if the calcination step is assumed to occur in one hour, the products of the calcination reactions are assumed to be released uniformly over the one-hour time period.

The mixture off-gas temperature during the drying stage is calculated using constant specific heats of $0.4787 \mathrm{cal} / \mathrm{g}-\mathrm{C}$ for water and $0.252 \mathrm{cal} / \mathrm{g}-\mathrm{C}$ for air. The drying stage is the only gas mixing process where a significant amount of water is added to the off-gas. On a mass basis, most gaseous species other than water such as air, $\mathrm{CO}$ and $\mathrm{CO}_{2}$ have similar heat capacities. Therefore, during the other melter phases the mixing off-gases are all simply treated as air. 
Table 3. Vitrification Process Steps

\begin{tabular}{|c|c|c|}
\hline Step & Description & Comment \\
\hline & Precipitation/Decant & \\
\hline 43 & $\begin{array}{l}\text { Product of pre-treatment processing in Tank } \\
17.3 \mathrm{E}\end{array}$ & Solution used for melter feed preparation \\
\hline 44 & Oxalic acid solution added to precipitator & $\begin{array}{l}8 \mathrm{wt} \% \text { oxalic acid added with } 0.3 \text { molar } \\
\text { excess over stochiometric requirement }\end{array}$ \\
\hline 45 & $\begin{array}{l}\text { Precipitator composition after oxalic acid addition } \\
\text { and conversion from nitrate to oxalate salts }\end{array}$ & $\begin{array}{l}\text { Total mixed contents of precipitator after } \\
\text { oxalate salt precipitation }\end{array}$ \\
\hline 46 & $\begin{array}{l}\text { Precipitate layer formed in precipitator by oxalic } \\
\text { acid addition }\end{array}$ & Volume calculated from 0.5 liter/mole [4] \\
\hline 47 & $\begin{array}{l}\text { Supernate layer formed in precipitator after } \\
\text { settling }\end{array}$ & Composition based on salt solubility \\
\hline 48 & $\begin{array}{l}\text { Decanted precipitate and remaining supernate in } \\
\text { precipitator }\end{array}$ & $\begin{array}{l}\text { Assumes } 1.5 \text { liters supernate remains in } \\
\text { precipitator for each batch }\end{array}$ \\
\hline \multirow[t]{2}{*}{49} & Supernate decanted from precipitator & \\
\hline & Precipitate Washing & \\
\hline 50 & Wash solution added to precipitator & $0.1 \mathrm{M}$ oxalic acid \\
\hline 51 & $\begin{array}{l}\text { Combined decanted slurry and oxalic acid wash } \\
\text { in precipitator before separation }\end{array}$ & Total mixed contents of precipitator \\
\hline 52 & $\begin{array}{l}\text { Precipitate layer formed in precipitator after } \\
\text { oxalic acid wash addition }\end{array}$ & Volume calculated from 0.5 liter/mole [4] \\
\hline 53 & $\begin{array}{l}\text { Supernate layer formed in precipitator after oxalic } \\
\text { acid wash addition }\end{array}$ & Composition based on salt solubility \\
\hline 54 & $\begin{array}{l}\text { Decanted precipitate and remaining supernate } \\
\text { after oxalic acid washing }\end{array}$ & $\begin{array}{l}\text { Assumes that } 1.5 \text { liters supernate remains } \\
\text { in precipitator after each batch }\end{array}$ \\
\hline 55 & $\begin{array}{l}\text { Supernate decanted from precipitator after oxalic } \\
\text { acid washing }\end{array}$ & \\
\hline \multirow[t]{2}{*}{56} & $\begin{array}{l}\text { Combined supernate decanted from precipitator } \\
\text { after precipitation and washing }\end{array}$ & Stream $49+$ Stream 55 \\
\hline & Melter Feed and Glass Product & \\
\hline 57 & $\begin{array}{l}\text { Deionized water used to rinse precipitator after } \\
\text { transfer of contents into melter }\end{array}$ & $270 \mathrm{ml}$ of deionized water \\
\hline 58 & $\begin{array}{l}\text { Combination of decanted slurry and rinse solution } \\
\text { representing total material fed to glass melter }\end{array}$ & \\
\hline 59 & Melter feed composition as equivalent oxides & \\
\hline 60 & Glass cullet composition & Grams and wt $\%$ oxides of $25 \mathrm{Sr}$ ABS-F \\
\hline 61 & Glass product composition & Grams and $\mathrm{wt} \%$ oxides of $\mathrm{Am} / \mathrm{Cm}$ glass \\
\hline
\end{tabular}


Table 3. Vitrification Process Steps (Continued)

\begin{tabular}{|c|c|c|}
\hline Step & Description & Comment \\
\hline & Melter Off-Gas: Drying Phase & \\
\hline 62 & $\begin{array}{l}\text { Entrainment in melter off-gas and water from } \\
\text { melter feed }\end{array}$ & $\begin{array}{l}\text { Assumes } 0.1 \% \text { of melter feed is entrained } \\
\text { in off-gas flow during drying phase }\end{array}$ \\
\hline 63 & $\begin{array}{l}\text { Sweep air passing over melter during drying } \\
\text { stage }\end{array}$ & $\begin{array}{l}\text { Sweep air flow set to } 65 \mathrm{scfm} \text { based on } \\
\text { TNX testing }\end{array}$ \\
\hline 64 & Combination of streams 62 and 63. & \\
\hline 65 & $\begin{array}{l}\text { Dilution air added to off-gas stream to prevent } \\
\text { condensation. (optional) }\end{array}$ & $\begin{array}{l}\text { Dilution air flow calculated to give } 70 \% \\
\text { relative humidity in stream } 66[6,7]\end{array}$ \\
\hline 66 & Combination of streams 64 and 65. & $\begin{array}{l}\text { Mixture temperature calculated by heat } \\
\text { balance using } c_{p}\left(\mathrm{H}_{2} \mathrm{O}\right)=0.4787 \mathrm{cal} / \mathrm{g}-\mathrm{C} \\
\text { and } \mathrm{c}_{\mathrm{p}} \text { (air) }=0.252 \mathrm{cal} / \mathrm{g}-\mathrm{C}\end{array}$ \\
\hline 67 & Motive air added to off-gas stream at exhaust jet. & $\begin{array}{l}\text { Motive air flow set equal to sum of sweep } \\
\text { air flow and dilution air flow }\end{array}$ \\
\hline \multirow[t]{2}{*}{68} & Combination of streams 66 and 67 & \\
\hline & Melter Off-Gas: Calcination Phase & \\
\hline 69 & Calcination reaction products & Oxidation reactions \\
\hline 70 & $\begin{array}{l}\text { Sweep air passing over melter during calcination } \\
\text { stage }\end{array}$ & $\begin{array}{l}\text { Sweep air flow set to } 65 \mathrm{scfm} \text { based on } \\
\text { TNX testing }\end{array}$ \\
\hline 71 & Combination of streams 69 and 70 & \\
\hline 72 & $\begin{array}{l}\text { Dilution air added to off-gas stream to prevent } \\
\text { condensation (optional) }\end{array}$ & $\begin{array}{l}\text { Dilution air flow calculated to give } 70 \% \\
\text { relative humidity in stream } 73[6,7]\end{array}$ \\
\hline 73 & Combination of streams 71 and 72 & \\
\hline 74 & Motive air added to off-gas stream at exhaust jet & $\begin{array}{l}\text { Motive air flow set equal to sum of sweep } \\
\text { air flow and dilution air flow }\end{array}$ \\
\hline \multirow[t]{2}{*}{75} & Combination of streams 73 and 74 & \\
\hline & Melter Off-Gas: Vitrification Phase & \\
\hline 76 & Oxygen released during vitrification & Reduction of $\mathrm{Ce}^{\mathrm{IV}}$ to $\mathrm{Ce} \mathrm{e}^{\mathrm{III}}$ \\
\hline 77 & $\begin{array}{l}\text { Sweep air passing over melter in vitrification } \\
\text { stage }\end{array}$ & $\begin{array}{l}\text { Sweep air flow set to } 65 \mathrm{scfm} \text { based on } \\
\text { TNX testing }\end{array}$ \\
\hline 78 & Combination of streams 76 and 77 & \\
\hline 79 & $\begin{array}{l}\text { Dilution air added to off-gas stream to prevent } \\
\text { condensation (optional) }\end{array}$ & $\begin{array}{l}\text { Dilution air flow calculated to give } 70 \% \\
\text { relative humidity in stream } 80[6,7]\end{array}$ \\
\hline 80 & Combination of streams 78 and 79 & \\
\hline 81 & Motive air added to off-gas stream at exhaust jet & $\begin{array}{l}\text { Motive air flow set equal to sum of sweep } \\
\text { air flow and dilution air flow }\end{array}$ \\
\hline 82 & Combination of streams 80 and 81 & \\
\hline
\end{tabular}


Table 4. Pretreatment and Vitrification Process Air Purges

\begin{tabular}{|l|ll|}
\hline Step & \multicolumn{1}{|c|}{ Description } & \multicolumn{1}{c|}{ Comment } \\
\hline & Tank Air Purges & Air purge calculations are based on maximum \\
\hline 83 & Air purge to Tank 17.1 & hydrogen gas generation in each tank. \\
84 & Air purge to Tank 16.1E & \\
85 & Air purge to Tank 16.3 & \\
86 & Air purge to Tank 17.3E & \\
87 & Air purge to melter feed tank & Total air purge requirements for pretreatment \\
88 & Air purge to melter batch tank & and vitrification processing steps. \\
89 & Air purge to precipitator & \\
90 & Combination of streams 83 through 89 & \\
\end{tabular}

\section{RESULTS}

Full results from the vitrification material balance calculations are presented in the two Appendices attached to this report. These pages were printed directly from the material balance spreadsheet. Appendix A shows a material balance carrying all of the Am/Cm material through each step in the vitrification process. These tables report the total amounts of glass product and off-gases released. Appendix B shows a material balance for the melter feed preparation and vitrification parts of the flowsheet using a 6.91 liter batch of feed material from tank 17.3. The 6.91 liter batch is the batch size that has been used for full scale testing at TNX and the batch size that will be processed in the F-Canyon operations to produce canisters of glass. In all of the spreadsheets, dark shading is used to indicate cells where fixed parameter values have been entered. Light shading is used in the spreadsheets to indicate cells where values have been adjusted to produce the target values in the lightly shaded cells that the attached arrows point to.

Stream 43 is the final product of the pre-treatment processing collected in the 17.3 evaporator that will serve as material for the melter feed preparation process. The material balance shows 1197 liters of feed solution in Tank 17.3 (stream 43) which at 6.91 liters/batch translates into 173.23 melter feed batches. In Appendix A, all of this material is shown in each remaining stage of the process. In Appendix B, stream 43 is reduced to a 6.91 liter batch of the solution in 17.3 which is then passed through the melter feed preparation and vitrification stages of the processes. Beyond stream 43 in Appendix B, the calculations are all based on the mass and composition of this 6.91 liter batch.

Stream 61 gives the composition of the projected glass product in total grams of each metal oxide assuming a $49 \%$ loading from the actinide and lanthanide oxides in the feed stream. The glass cullet used is type 25SrABS-F with the composition specified by Meaker [8]. At the bottom of column 61, we see that about $320 \mathrm{~kg}$ of glass are predicted to be produced from the projected melter feed supply. The mass summation at the bottom of column 61 in Appendix B, shows that a 6.91 liter batch of the projected melter feed will produce $1.85 \mathrm{~kg}$ of glass. Column 61a gives the glass product composition as weight percent oxides. 
Assuming that $0.10 \%$ of the total oxides in the melter feed are entrained in the off-gas flow, the top section of stream 62 shows the grams of each oxide that is lost from the melter through entrainment. The lower section of stream 62 shows the total grams of water produced from the melter drying step. Streams 69 and 76 show the total grams of gas produced in the melter calcination and vitrification steps, respectively. Streams 65, 72 and 79 on Spreadsheet pages 25, 27 and 29 show the dilution air that must be added to the combined melter off-gas and sweep air stream to give a mixture relative humidity of 70\%. To calculate the required dilution airflow, a supply temperature of $20{ }^{\circ} \mathrm{C}$ was assumed. Trial calculations showed that colder dilution air would more readily condense water vapor and was therefore the most conservative design case. Streams 66, 73 and 80 show combined melter off-gas flows obtained by adding the gaseous products from the melter operations with the dilution air. As an alternative to adding dilution air to the melter off-gas, Streams 66, 73 and 80 on Spreadsheet pages 26, 28 and 30 show the temperature to which the combined melter off-gas and sweep air stream must be heated to reduce the relative humidity to $70 \%$. During the drying stage, when most of the water is released from the melter, the calculation shows that a temperature of about $37{ }^{\circ} \mathrm{C}$ is required to meet the $70 \%$ relative humidity criterion. During the other phases of the melter operation, an off-gas temperature of only about $26^{\circ} \mathrm{C}$ is needed. An estimate of the humidity $(700 \mathrm{ppm})$ in the F Canyon process air supply was obtained from SOP 221-F-62042. The motive airflow in the off-gas system was set equal to the sum of the melter off-gas, sweep air and dilution airflow. All of the combined gas streams show the material flow in $\mathrm{g} / \mathrm{hr}$ assuming that the melter gases evolve over a one hour time period.

\section{REFERENCES}

1. Smith, III, F. G., "Am/Cm Vitrification Process: Pretreatment Material Balance Calculations (U)," WSRC-TR-2000-00219, Rev. 1, December 12, 2000.

2. Choi, A. S., "Design Basis Material Balance for Americium/Curium Vitrification Flowsheet," SRT-AMC-96-0018, June 11, 1996.

3. Rudisill, T. S., "Pretreatment of Americium/Curium Solutions for Vitrification (U)," WSRC-TR96-0074, March 1996.

4. Beck, S. B., "Am/Cm Oxalate Precipitation and Washing Demonstration," WSRC-TR-96-0116, June 11, 1996.

5. Marek, J. C., "Hydrogen Generation from Am/Cm Vessels (U),” S-CLC-F-00196, July, 11, 1997.

6. Zamecnik, J. R., “Am/Cm Offgas System Design Bases,” SRT-AMC-99-0134, Rev. 0, June 28, 1999.

7. Zamecnik, J. R. and Smith, F. G., "Evaluation of Am/Cm Offgas Treatment System Design Bases," SRT-GFM-99-0019, Rev. 0, June 28, 1999.

8. Meaker, T. F., Spreadsheet page transmitted to Doug Witt dated 10-29-1996. 
WSRC-TR-2000-00218, Revision 1

Page 13 of 42

APPENDIX A

Am/Cm Material Balance With Total Material in Streams Melter Feed Preparation Steps and Melter Operations 
WSRC-TR-2000-00218

Revision 1

Page 14 of 42

Am/Cm Material Balance Spreadsheet Page 17

Version 2

\begin{tabular}{|c|c|c|c|c|c|c|c|c|c|}
\hline \multirow{2}{*}{$\begin{array}{l}\text { Batch } \\
\text { Stream Name } \\
\text { Units } \\
\text { Stream Number }\end{array}$} & \multicolumn{9}{|l|}{ Precipitation } \\
\hline & $\begin{array}{c}\text { Batch } \\
\text { Tank } \\
\text { grams } \\
43 \\
\end{array}$ & $\begin{array}{c}\text { MPPF } \\
\text { Feed } \\
y / \\
43 \\
\end{array}$ & $\begin{array}{c}\text { Oxalic Acid } \\
8 \text { wt\% } \\
\text { grams } \\
44 \\
\end{array}$ & $\begin{array}{c}\text { Converted } \\
\text { Compositlon } \\
\text { grams } \\
45 \\
\end{array}$ & $\begin{array}{l}\text { Precipitate } \\
\text { grams } \\
46\end{array}$ & $\begin{array}{c}\text { Supernate } \\
\text { in Tank } \\
\text { grams } \\
47 \\
\end{array}$ & $\begin{array}{c}\text { Decanted } \\
\text { Slurry } \\
\text { grams } \\
48 \\
\end{array}$ & $\begin{array}{c}\text { Decant } \\
\text { Collection } \\
\text { grams } \\
49 \\
\end{array}$ & $\begin{array}{l}\text { Percent } \\
\text { Recovery }\end{array}$ \\
\hline Lan salts & & & & & & & & & \\
\hline $\mathrm{La}(\mathrm{NO}) 3$ & $3.431 E+04$ & $2.866 \mathrm{E}+01$ & ZERO & ZERO & ZERO & ZERO & ZERO & ZERO & \\
\hline $\mathrm{Ce}(\mathrm{NO} 3)_{3} 3$ & $2.689 E+04$ & $2.247 \mathrm{E}+01$ & ZERO & ZERO & ZERO & ZERO & ZERO & ZERO & \\
\hline $\operatorname{Pr}(\mathrm{NO}) 3$ & 2.297E+04 & $1.919 \mathrm{E}+01$ & ZERO & ZERO & ZERO & ZERO & ZERO & ZERO & \\
\hline $\mathrm{Nd}(\mathrm{NO}) 3$ & $6.517 \mathrm{E}+04$ & $5.444 E+01$ & ZERO & ZERO & ZERO & ZERO & ZERO & ZERO & \\
\hline $\mathrm{Sm}(\mathrm{NO}) 3$ & $1.505 E+04$ & $1.257 E+01$ & ZERO & ZERO & ZERO & ZERO & ZERO & ZERO & \\
\hline $\mathrm{Eu}(\mathrm{NO}) 3$ & $3.046 E+03$ & $2.545 \mathrm{E}+00$ & ZERO & ZERO & ZERO & ZERO & ZERO & ZERO & \\
\hline $\mathrm{Gd}(\mathrm{NO}) 3$ & $7.595 E+03$ & $6.345 E+00$ & ZERO & ZERO & ZERO & ZERO & ZERO & ZERO & \\
\hline $\mathrm{Tb}(\mathrm{NO}) 3$ & $9.555 E+02$ & 7.982E-01 & ZERO & ZERO & ZERO & ZERO & ZERO & ZERO & \\
\hline $\mathrm{Dy}(\mathrm{NO3}) 3$ & $9.439 E+02$ & 7.885E-01 & ZERO & ZERO & ZERO & ZERO & ZERO & ZERO & \\
\hline $\mathrm{Ho}(\mathrm{NO}) 3$ & $9.363 \mathrm{E}+02$ & $7.822 \mathrm{E}-01$ & ZERO & ZERO & ZERO & ZERO & ZERO & ZERO & \\
\hline $\operatorname{Er}(\mathrm{NO}) 3$ & $9.292 E+02$ & $7.762 \mathrm{E}-01$ & ZERO & ZERO & ZERO & ZERO & ZERO & ZERO & \\
\hline $\operatorname{Tm}(\mathrm{NO})_{3} 3$ & $1.244 E+03$ & $1.039 E+00$ & ZERO & ZERO & ZERO & ZERO & ZERO & ZERO & \\
\hline $\mathrm{Yb}(\mathrm{NO}) 3$ & $9.124 E+02$ & $7.622 E-01$ & ZERO & ZERO & ZERO & ZERO & ZERO & ZEFO & \\
\hline $\begin{array}{l}\text { Lu(NO3)3 } \\
\text { Lan oxalates }\end{array}$ & $9.070 \mathrm{E}+02$ & 7.577E-01 & ZERO & ZERO & ZERO & ZERO & ZERO & ZERO & \\
\hline $\mathrm{La2}(\mathrm{C} 2 \mathrm{O} 4) 3$ & ZERO & ZERO & ZERO & $2.861 E+04$ & $2.483 E+04$ & $3.776 \mathrm{E}+03$ & $2.515 E+04$ & $3.462 E+03$ & 87.90 \\
\hline $\mathrm{Ce} 2\left(\mathrm{C}_{2} \mathrm{O} 4\right) 3$ & ZERO & ZERO & ZERO & $2.244 E+04$ & $2.145 E+04$ & $9.936 E+02$ & $2.153 E+04$ & $9.110 E+02$ & 95.94 \\
\hline $\begin{array}{l}\mathrm{Pr}(\mathrm{C} 2 \mathrm{O} 4) 3 \\
\mathrm{Nd} 2(\mathrm{C} 2 \mathrm{O} 4) 3\end{array}$ & $\begin{array}{l}\text { ZERO } \\
\text { ZERO }\end{array}$ & $\begin{array}{l}\text { ZERO } \\
\text { ZERO }\end{array}$ & $\begin{array}{l}\text { ZERO } \\
\text { ZERO }\end{array}$ & $\begin{array}{l}1.918 \mathrm{E}+04 \\
5.452 \mathrm{E}+04\end{array}$ & $\begin{array}{l}1.865 E+04 \\
5.398 E+04\end{array}$ & $\begin{array}{l}5.298 \mathrm{E}+02 \\
5.321 \mathrm{E}+02\end{array}$ & $\begin{array}{l}1.869 \mathrm{E}+04 \\
5.403 \mathrm{E}+04\end{array}$ & $\begin{array}{l}4.857 E+02 \\
4.878 E+02\end{array}$ & $\begin{array}{l}97.47 \\
99.11\end{array}$ \\
\hline $\mathrm{Sm} 2(\mathrm{C} 2 \mathrm{O} 4) 3$ & ZERO & ZERO & ZERO & $1.264 E+04$ & $1.213 E+04$ & $5.033 E+02$ & $1.217 E+04$ & $4.615 E+02$ & 96.35 \\
\hline Eu2(C2O4)3 & ZERO & ZERO & ZERO & $2.560 \mathrm{E}+03$ & $2.518 E+03$ & $4.179 E+01$ & $2.521 E+03$ & $3.831 \mathrm{E}+01$ & 98.50 \\
\hline $\mathrm{Gd} 2\left(\mathrm{C}_{2} \mathrm{O} 4\right) 3$ & ZERO & ZERO & ZERO & $6.401 E+03$ & $6.373 E+03$ & $2.771 E+01$ & $6.375 E+03$ & $2.541 E+01$ & 99.60 \\
\hline $\mathrm{Tb} 2\left(\mathrm{C}_{2} \mathrm{O} 4\right)_{3}$ & ZERO & ZERO & ZERO & $8.060 E+02$ & $5.552 E+02$ & $2.508 \mathrm{E}+02$ & $5.761 E+02$ & $2.299 E+02$ & 71.47 \\
\hline Dy2 $\left(\mathrm{C}_{2} \mathrm{O} 4\right) 3$ & ZERO & ZERO & ZERO & $7.977 \mathrm{E}+02$ & $5.469 \varepsilon+02$ & $2.508 E+02$ & $5.678 E+02$ & $2.299 \mathrm{E}+02$ & 71.18 \\
\hline $\mathrm{Ho} 2\left(\mathrm{C}_{2} \mathrm{O} 4\right) 3$ & ZERO & ZERO & ZERO & $7.922 E+02$ & $5.415 \varepsilon+02$ & $2.508 \mathrm{E}+02$ & $5.623 E+02$ & $2.299 \mathrm{E}+02$ & 70.98 \\
\hline $\mathrm{E}-2(\mathrm{C} 2 \mathrm{O} 4)^{3}$ & ZERO & ZEAO & ZERO & $7.872 E+02$ & $5.364 E+02$ & $2.508 \mathrm{E}+02$ & $5.573 E+02$ & $2,299 E+02$ & 70.79 \\
\hline $\mathrm{Tm} 2\left(\mathrm{C}_{2} \mathrm{O}_{4}\right) 3$ & ZERO & ZERO & ZERO & $1.054 E+03$ & $8.037 \mathrm{E}+02$ & $2.508 E+02$ & $8.245 E+02$ & $2.299 \mathrm{E}+02$ & 78.20 \\
\hline $\mathrm{Yb} 2\left(\mathrm{C}_{2} \mathrm{O} 4\right) 3$ & ZERO & ZERO & ZERO & $7.752 E+02$ & $5.244 E+02$ & $2.508 E+02$ & $5.453 \mathrm{E}+02$ & $2.299 \mathrm{E}+02$ & 70.34 \\
\hline $\mathrm{Lu} 2(\mathrm{C} 2 \mathrm{O} 4) 3$ & ZERO & ZERO & ZERO & $7.714 \mathrm{E}+02$ & $5.206 \mathrm{E}+02$ & $2.50 \mathrm{BE}+02$ & $5.415 E+02$ & $2.299 \mathrm{E}+02$ & 70.19 \\
\hline Act salts & & & & & & & & & \\
\hline $\mathrm{Am}(\mathrm{NO} 3)_{3}$ & $1.566 \mathrm{E}+04$ & $1.308 E+01$ & ZERO & ZERO & ZERO & ZERO & ZERO & ZERO & \\
\hline $\mathrm{Cm}(\mathrm{NO}) 3$ & $3.843 E+03$ & $3.210 E+00$ & ZERO & ZERO & ZERO & ZERO & ZERO & ZERO & \\
\hline $\mathrm{NpO} 2$ (NÓ3) & $6.289 \mathrm{E}-01$ & $5.253 E-04$ & ZERO & ZERO & ZERO & ZERO & ZERO & ZERO & \\
\hline $\mathrm{Pu}(\mathrm{NO}) 4$ & $4.400 E+03$ & $3.676 E+\infty 0$ & ZERO & ZERO & ZERO & ZERO & ZERO & ZERO & \\
\hline UO2(NO3)2 & $2.635 E+02$ & $2.201 \mathrm{E} \cdot 01$ & ZERO & ZERO & ZERO & ZERO & ZERO & ZERO & \\
\hline CsNO3 & $1.360 \mathrm{E}+00$ & $1.136 \mathrm{E}-03$ & ZERO & ZERO & ZERO & ZERO & ZERO & ZERO & \\
\hline $\begin{array}{l}\text { Act oxalates } \\
\text { Am2(C2O4)3 }\end{array}$ & ZERO & ZERO & ZERO & $1.369 E+04$ & $-1.367 E+04$ & $2.547 E+01$ & $1.367 E+04$ & $2.335 \mathrm{E}+01$ & 99.83 \\
\hline $\mathrm{Cm} 2\left(\mathrm{C}_{2} \mathrm{O} 4\right) 3$ & ZERO & ZERO & ZERO & $3.360 E+03$ & $3.345 \mathrm{E}+03$ & $1.537 E+01$ & $3.346 E+03$ & $1.409 \mathrm{E}+01$ & 99.58 \\
\hline $\mathrm{Np}_{2} \mathrm{O}_{4}\left(\mathrm{C}_{2} \mathrm{O} 4\right)$ & ZERO & ZERO & ZERO & $5.947 \mathrm{E}-01$ & $0.000 E+00$ & $5.947 \mathrm{E}-01$ & $4.943 \mathrm{E}-02$ & 5.452E-01 & 8.31 \\
\hline $\mathrm{Pu}\left(\mathrm{C}_{2} \mathrm{O} 4\right) 2$ & ZERO & ZERO & ZERO & $3.751 E+03$ & $3.733 \mathrm{E}+03$ & $1.798 E+01$ & $3.735 E+03$ & $1.648 E+01$ & 99.56 \\
\hline $\mathrm{UO2}(\mathrm{C} 2 \mathrm{O} 4)$ & ZERO & ZERO & ZERO & $2.394 E+02$ & $0.000 E+00$ & $2,394 E+02$ & $1.990 E+01$ & $2.195 \mathrm{E}+02$ & 8.31 \\
\hline $\mathrm{Cs}_{2} \mathrm{C}_{2} \mathrm{O}_{4}$ & ZERO & ZERO & ZERO & $1.234 E+\infty 0$ & $0.000 E+\infty$ & $1.234 E+00$ & $1.026 \mathrm{E}-01$ & $1.132 E+00$ & 8.31 \\
\hline
\end{tabular}


WSRC-TR-2000-00218

Revision 1

Page 15 of 42

Am/Cm Material Balance Spreadsheet Page 18

Version 2

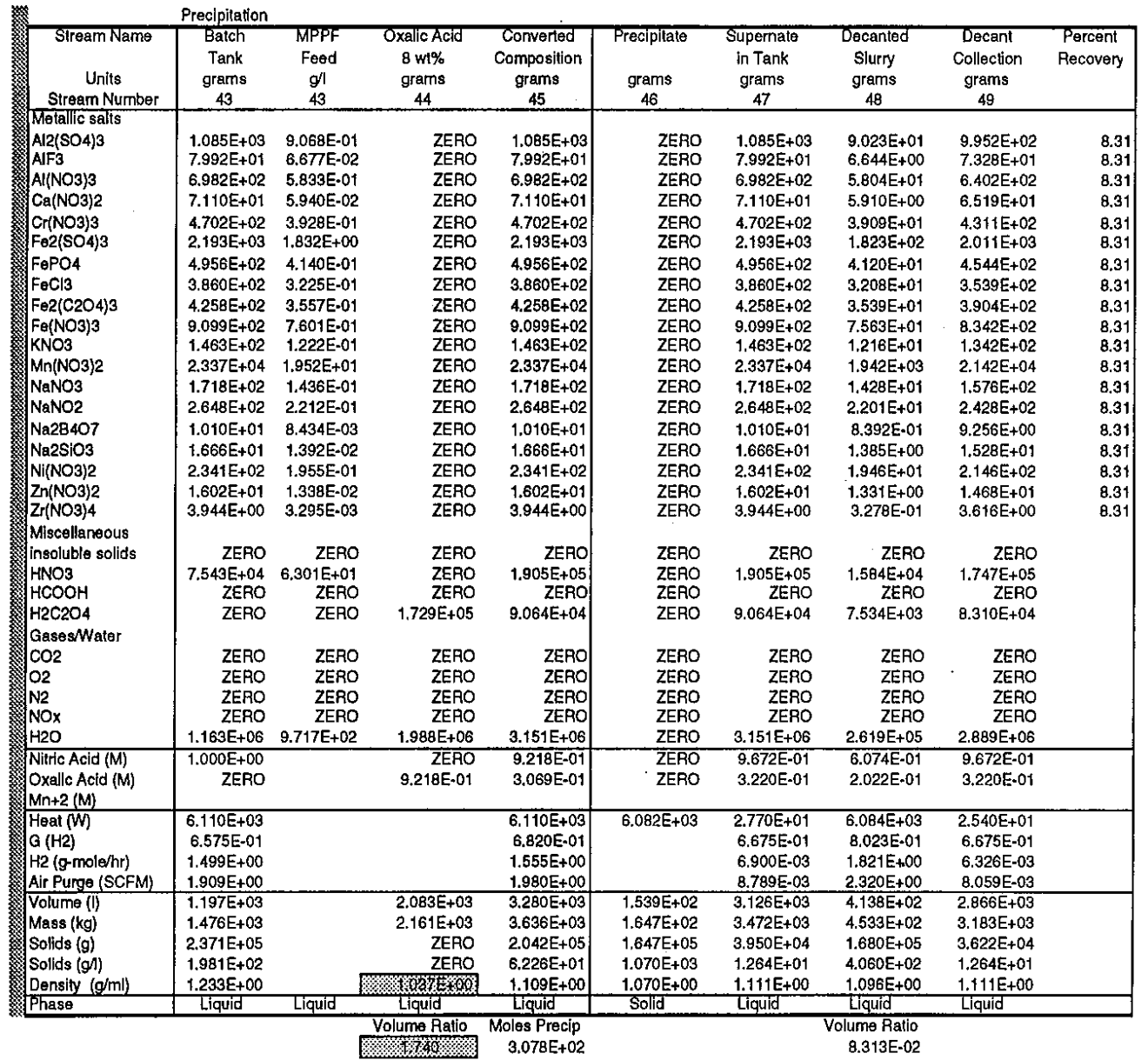


WSRC-TR-2000-00218

Revision 1

Page 16 of $42 \quad$ Am/Cm Material Balance Spreadsheet Page 19

Version 2

\begin{tabular}{|c|c|c|c|c|c|c|c|}
\hline $\begin{array}{c}\text { Stream Name } \\
\text { Units } \\
\text { Stream Number }\end{array}$ & $\begin{array}{l}\text { Oxalic Acid } \\
0.1 \mathrm{M} \\
\text { grams } \\
50\end{array}$ & $\begin{array}{c}\text { Slurry } \\
\text { and Wash } \\
\text { grams } \\
51\end{array}$ & $\begin{array}{l}\text { Precipitale } \\
\text { grams } \\
52\end{array}$ & $\begin{array}{c}\text { Supernate } \\
\text { in Tank } \\
\text { grams } \\
53\end{array}$ & $\begin{array}{l}\text { Decanted } \\
\text { Slurry } \\
\text { grams } \\
54\end{array}$ & $\begin{array}{l}\text { Decant } \\
\text { Collection } \\
\text { grams } \\
55\end{array}$ & $\begin{array}{l}\text { Percent } \\
\text { Recovery }\end{array}$ \\
\hline Lan salts & & & & & & & \\
\hline $\mathrm{La}(\mathrm{NO}) 3$ & ZERO & ZERO & ZERO & ZERO & ZERO & ZEAO & \\
\hline $\mathrm{Ce}(\mathrm{NO})^{3}$ & ZERO & ZERO & ZERO & ZERO & ZERO & ZERO & \\
\hline $\operatorname{Pr}(\mathrm{NO}) 3$ & ZERO & ZERO & ZERO & ZERO & ZERO & ZERO & \\
\hline $\mathrm{Nd}(\mathrm{NO}) 3$ & ZERO & ZERO & ZERO & ZERO & ZERO & ZERO & \\
\hline $\mathrm{Sm}(\mathrm{NO}) 3$ & ZERO & ZERO & ZERO & ZERO & ZERO & ZERO & \\
\hline Eu(NO3) 3 & ZERO & ZERO & ZERO & ZERO & ZERO & ZERO & \\
\hline Gd(NO3) 3 & ZERO & ZERO & ZERO & ZERO & ZERO & ZERO & \\
\hline $\mathrm{Tb}(\mathrm{NO})^{3} 3$ & ZERO & ZERO & ZERO & ZERO & ZERO & ZERO & \\
\hline Dy $(\mathrm{NO}) 3$ & ZERO & ZERO & ZERO & ZERO & ZERO & ZERO & \\
\hline $\mathrm{Ho}(\mathrm{NO}) 3$ & ZERO & ZERO & ZERO & ZERO & ZERO & ZERO & \\
\hline Er(NO3)3 & ZERO & ZERO & ZERO & ZEPO & ZERO & ZERO & \\
\hline $\operatorname{Tm}(\mathrm{NO}) 3$ & ZERO & ZERO & ZERO & ZERO & ZERO & ZERO & \\
\hline $\mathrm{Yb}(\mathrm{NO}) 3$ & ZERO & ZERO & ZEAO & ZERO & ZERO & ZERO & \\
\hline Lu(NO3)3 & ZERO & ZERO & ZERO & ZERO & ZERO & ZERO & \\
\hline Lan $(\mathrm{C} 2 \mathrm{O}) \mathrm{4}) \mathrm{B}$ & ZERO & $2.515 E+04$ & $2.515 E+04$ & $1.273 E+\infty 0$ & $2,515 E+04$ & $1.041 E+00$ & 87.90 \\
\hline $\mathrm{C}_{2} 2\left(\mathrm{C}_{2} \mathrm{O} 4\right) 3$ & ZERO & $2.153 E+04$ & $2.153 E+04$ & B.117E-01 & $2.153 E+04$ & $6.637 \mathrm{E}-01$ & 95,94 \\
\hline $\begin{array}{l}\mathrm{Pr} 2(\mathrm{C} 2 \mathrm{O} 4) 3 \\
\mathrm{Nd}(\mathrm{C} 2 \mathrm{O}) 3\end{array}$ & $\begin{array}{l}\text { ZERO } \\
\text { ZERO }\end{array}$ & $\begin{array}{l}\text { 1. } 869 E+04 \\
5.403 E+04\end{array}$ & $\begin{array}{l}1.869 E+04 \\
5.403 E+04\end{array}$ & 2.407E-01 & $\begin{array}{l}1.869 \mathrm{E}+04 \\
5.403 \mathrm{E}+04\end{array}$ & $\begin{array}{l}1.968 \mathrm{E}-01 \\
3012 \mathrm{E}-01\end{array}$ & 97.47 \\
\hline $\mathrm{Sm} 2\left(\mathrm{C}_{2} \mathrm{O} 4\right)_{3}$ & ZERO & $1.217 E+04$ & $1.217 E+04$ & $3.677 \mathrm{E} .01$ & $1.217 E+04$ & 3.006E-01 & 96.35 \\
\hline Eu2(C2O4)3 & ZERO & $2.521 E+03$ & $2.521 E+03$ & $3.811 \mathrm{E}-02$ & $2.521 \mathrm{E}+03$ & 3.117E-02 & 98.50 \\
\hline $\mathrm{Gd2}(\mathrm{C} 2 \mathrm{O} 4)_{3}$ & ZERO & $6.375 E+03$ & $6.375 \mathrm{E}+03$ & 4.809E-02 & $6.375 \mathrm{E}+03$ & $3.932 \mathrm{E} \cdot 02$ & 99.60 \\
\hline $\mathrm{Tb} 2(\mathrm{C} 2 \mathrm{O} 4)_{3}$ & ZERO & $5.761 E+02$ & $5.759 E+02$ & 2.048E-01 & $5.759 E+02$ & $1.675 \mathrm{E}-01$ & 71.45 \\
\hline Dy2(C2O4) 3 & ZERO & $5.678 E+02$ & $5.676 E+02$ & $2.048 \mathrm{E} \cdot 01$ & $5.676 \mathrm{E}+02$ & $1.675 \mathrm{E}-01$ & 71.16 \\
\hline $\mathrm{Ho} 2(\mathrm{C} 2 \mathrm{O} 4) 3$ & ZERO & $5.623 E+02$ & $5.621 E+02$ & $2.048 \mathrm{E}-0 t$ & $5.622 \mathrm{E}+02$ & $1.675 \mathrm{E}-01$ & 70.96 \\
\hline $\mathrm{E}-2\left(\mathrm{C}_{2} \mathrm{O} 4\right)^{3}$ & ZERO & $5.573 E+02$ & $5.571 E+02$ & 2.048E-01 & $5.571 \mathrm{E}+02$ & $1.675 \mathrm{E}-01$ & 70.77 \\
\hline $\operatorname{Tm} 2(\mathrm{C} 2 \mathrm{O} 4) 3$ & ZERO & $8.245 E+02$ & $8.243 E+02$ & 2.048E-01 & $8.243 E+02$ & $1.675 \mathrm{E}-01$ & 78.18 \\
\hline $\mathrm{Yb} 2(\mathrm{C} 2 \mathrm{O} 4)^{3}$ & ZERO & $5.453 E+02$ & $5.451 E+02$ & $2.048 \mathrm{E} \cdot 01$ & $5.451 \mathrm{E}+02$ & $1.675 \mathrm{E}-01$ & 70.32 \\
\hline $\mathrm{Lu} 2(\mathrm{C} 2 \mathrm{O} 4)^{3}$ & ZERO & $5.415 \mathrm{E}+02$ & $5.413 E+02$ & $2.048 \mathrm{E}-01$ & $5.413 E+02$ & $1.675 \mathrm{E}-01$ & 70.17 \\
\hline Act salts & & & & & & & \\
\hline $\mathrm{Am}(\mathrm{NO}) 3$ & ZERO & ZERO & ZERO & ZERO & ZERO & ZERO & \\
\hline $\mathrm{cm}(\mathrm{NO} 3)_{3}$ & ZERO & ZERO & ZERO & ZERO & ZERO & ZERO & . \\
\hline NpO2(NO3) & ZERO & ZERO & ZERO & ZERO & ZERO & ZERO & \\
\hline $\mathrm{Pu}(\mathrm{NO}){ }_{4}$ & ZERO & ZERO & ZERO & ZEFO & ZEFO & ZERO & \\
\hline $\mathrm{UO} 2(\mathrm{NO} 3) 2$ & ZERO & ZERO & ZERO & ZERO & ZERO & ZERO & \\
\hline CsNO3 & ZERO & ZERO & ZERO & ZERO & ZERO & ZERO & \\
\hline $\begin{array}{l}\text { Act oxalates } \\
\text { Am2 }\left(\mathrm{C} 2 \mathrm{O}_{4}\right)\end{array}$ & ZERO & $1.367 E+04$ & $1367 \mathrm{E}+04$ & $2.100 \mathrm{E}+00$ & $1367 \mathrm{E}+04$ & $1717 \mathrm{E}+00$ & 9982 \\
\hline $\mathrm{Cm} 2(\mathrm{C} 2 \mathrm{O} 4)_{3}$ & ZERO & $3.346 \mathrm{E}+03$ & $3.345 E+03$ & $1.051 E+\infty 0$ & $3.345 E+03$ & 8.595E-01 & 99.56 \\
\hline $\mathrm{Np2O} 4\left(\mathrm{C}_{2} \mathrm{O} 4\right)$ & ZERO & $4.943 \mathrm{E}-02$ & $0.000 E+00$ & $4.943 \mathrm{E}-02$ & $9.013 \mathrm{E}-03$ & $4,042 \mathrm{E} \cdot 02$ & 1.52 \\
\hline $\mathrm{Pu}\left(\mathrm{C}_{2} \mathrm{O} 4\right) 2$ & ZERO & $3.735 E+03$ & $3.713 E+03$ & $2.162 E+01$ & $3.717 E+03$ & $1.768 \mathrm{E}+01$ & 99.09 \\
\hline $\mathrm{UO} 2\left(\mathrm{C}_{2} \mathrm{O} 4\right)$ & ZERO & $1.990 E+01$ & $0.000 E+00$ & $1.990 E+01$ & $3.628 E+00$ & $1.627 \mathrm{E}+01$ & 1.52 \\
\hline $\mathrm{C}_{32} 2 \mathrm{C}_{2} \mathrm{O}_{4}$ & ZERO & $1.026 \mathrm{E}-01$ & $0.000 E+00$ & $1.026 \mathrm{E} \cdot 01$ & $1.871 \mathrm{E}-02$ & 8.389E-02 & 1.52 \\
\hline
\end{tabular}


WSRC-TR-2000-00218

Revision 1

\begin{tabular}{|c|c|c|c|c|c|c|c|}
\hline $\begin{array}{c}\text { Stream Name } \\
\text { Units } \\
\text { Stream Number }\end{array}$ & $\begin{array}{c}\text { Oxalic Acid } \\
0.1 \mathrm{M} \\
\text { grams } \\
50\end{array}$ & $\begin{array}{c}\text { Slurry } \\
\text { and Wash } \\
\text { grams } \\
51\end{array}$ & $\begin{array}{l}\text { Precipitalo } \\
\text { grams } \\
52\end{array}$ & $\begin{array}{c}\text { Supernate } \\
\text { in Tank } \\
\text { grams } \\
53\end{array}$ & $\begin{array}{l}\text { Decanted } \\
\text { Slurry } \\
\text { grams } \\
54\end{array}$ & $\begin{array}{l}\text { Decant } \\
\text { Collection } \\
\text { grams } \\
55\end{array}$ & $\begin{array}{l}\text { Percent } \\
\text { Recovery }\end{array}$ \\
\hline Metallic salts & & & & & & & \\
\hline $\begin{array}{l}\mathrm{Al2}(\mathrm{SO} 4) 3 \\
\mathrm{AlF3}\end{array}$ & $\begin{array}{l}\text { ZERO } \\
\text { ZERO }\end{array}$ & $\begin{array}{l}9.023 E+01 \\
6.644 E+00\end{array}$ & $\begin{array}{l}\text { ZERO } \\
\text { ZERO }\end{array}$ & $\begin{array}{l}9.023 \mathrm{E}+01 \\
6.644 \mathrm{E}+00\end{array}$ & $\begin{array}{l}1.645 E+01 \\
1.211 E+00\end{array}$ & $\begin{array}{l}7.378 \mathrm{E}+01 \\
5.432 \mathrm{E}+00\end{array}$ & $\begin{array}{l}1.52 \\
1.52\end{array}$ \\
\hline Al(NO3)3 & ZERO & $5.804 E+01$ & ZERO & $5.804 E+01$ & $1.058 E+01$ & $4.746 \mathrm{E}+01$ & 1.52 \\
\hline $\mathrm{Ca}(\mathrm{NO} 3)_{2}$ & ZERO & $5.910 E+00$ & ZERO & $5.910 \mathrm{E}+00$ & $1.078 E+00$ & $4.933 \mathrm{E}+00$ & 1.52 \\
\hline $\begin{array}{l}\mathrm{Cr}(\mathrm{NO} 3)_{3} \\
\mathrm{Fe} 2(\mathrm{SO} 4)_{3}\end{array}$ & $\begin{array}{l}\text { ZERO } \\
\text { ZERO }\end{array}$ & $\begin{array}{l}3.909 E+01 \\
1.823 E+02\end{array}$ & $\begin{array}{l}\text { ZERO } \\
\text { ZERO }\end{array}$ & $\begin{array}{l}3.909 E+01 \\
1.823 E+02\end{array}$ & $\begin{array}{l}7.127 E+00 \\
3.324 E+01\end{array}$ & $\begin{array}{l}3.196 \mathrm{E}+01 \\
1.491 \mathrm{E}+02\end{array}$ & $\begin{array}{l}1.52 \\
1.52\end{array}$ \\
\hline $\mathrm{FePO} 4$ & ZERO & 4. $120 E+01$ & ZERO & 4. $120 \mathrm{E}+01$ & $7.512 E+00$ & $3.369 E+01$ & 1.52 \\
\hline $\mathrm{FeCl3}$ & ZERO & $3.208 E+01$ & ZERO & $3.208 \mathrm{E}+01$ & $5.850 E+00$ & $2.623 E+01$ & 1.52 \\
\hline $\mathrm{Fe} 2(\mathrm{C} 2 \mathrm{O} 4)_{3}$ & ZERO & $3.539 E+01$ & ZERO & $3.539 E+01$ & $6.453 E+00$ & $2.894 E+01$ & 1.52 \\
\hline $\begin{array}{l}\mathrm{Fe}(\mathrm{NO} 3)_{3} \\
\mathrm{KNO} 3\end{array}$ & $\begin{array}{l}\text { ZERO } \\
\text { ZERO }\end{array}$ & $\begin{array}{l}7.563 E+01 \\
1216 E+01\end{array}$ & $\begin{array}{l}\text { ZERO } \\
\text { ZERO }\end{array}$ & $7.563 E+01$ & $\begin{array}{l}1.379 E+01 \\
2.318 E+00\end{array}$ & $6.184 E+01$ & $\begin{array}{l}1.52 \\
1.52\end{array}$ \\
\hline $\mathrm{Mn}(\mathrm{NO}) 2$ & ZERO & $1.942 E+03$ & ZERO & $1.942 E+03$ & $3.542 \mathrm{E}+02$ & $1.5 B B E+03$ & $\begin{array}{l}1.52 \\
1.52\end{array}$ \\
\hline $\mathrm{NaNO3}$ & ZERO & $1.428 E+01$ & ZERO & $1.428 \mathrm{E}+01$ & $2.605 E+00$ & $1.168 E+01$ & 1.52 \\
\hline NaNO2 & ZERO & $2.201 E+01$ & ZERO & $2.201 E+01$ & $4.013 E+00$ & $1.800 E_{+} 01$ & 1.52 \\
\hline $\mathrm{Na} 2 \mathrm{~B} 4 \mathrm{O} 7$ & ZERO & $8.392 E-01$ & ZERO & $8.392 \mathrm{E} \cdot 01$ & $1.530 \mathrm{E}-01$ & $6.862 E=01$ & 1.52 \\
\hline $\mathrm{Na2SiO3}$ & ZERO & $1,385 E+00$ & ZERO & $1.385 E+00$ & $2.525 \mathrm{E}-01$ & $1.132 \mathrm{E}+00$ & 1.52 \\
\hline $\mathrm{Ni}(\mathrm{NO} 3)_{2}$ & ZERO & $1.946 E+01$ & ZERO & $1.946 E+01$ & $3.548 E+00$ & $1.591 \mathrm{E}+01$ & 1.52 \\
\hline $\mathrm{Zn}(\mathrm{NO}) 2$ & ZERO & $1.331 E+00$ & ZERO & $1,331 E+00$ & $2.427 \mathrm{E}-01$ & $1,089 \mathrm{E}+00$ & 1.52 \\
\hline $\mathrm{Zr}(\mathrm{NO} 3)_{4}$ & ZERO & 3.27BE-01 & ZERO & $3.27 \mathrm{BE} \cdot 01$ & $5.977 \mathrm{E}-02$ & $2.681 \mathrm{E}-01$ & 1.52 \\
\hline Miscellaneous & & & & & & & \\
\hline insoluble solids & ZERO & ZERO & ZEFO & ZERO & ZERO & ZERO & \\
\hline $\begin{array}{l}\mathrm{HNOS} \\
\mathrm{HCOOH}\end{array}$ & $\begin{array}{l}\text { ZERO } \\
\text { ZERO }\end{array}$ & $\begin{array}{r}1.584 E+04 \\
\text { ZERO }\end{array}$ & $\begin{array}{l}\text { ZERO } \\
\text { ZERO }\end{array}$ & $\begin{array}{r}1.584 E+04 \\
\text { ZERO }\end{array}$ & $\begin{array}{r}2.888 E+03 \\
\text { ZERO }\end{array}$ & $\begin{array}{r}1.295 \mathrm{E}+04 \\
\text { ZERO }\end{array}$ & \\
\hline $\mathrm{H} 2 \mathrm{C}_{2} \mathrm{O} 4$ & $1.078 E+04$ & $1.831 E+04$ & ZERO & $1.831 E+04$ & $3.339 E+03$ & $1.497 \mathrm{E}+04$ & \\
\hline GasesWWater & & & & & & & \\
\hline $\mathrm{CO} 2$ & ZERO & ZERO & ZERO & ZERO & ZERO & ZERO & \\
\hline $\mathrm{O} 2$ & ZERO & ZERO & ZERO & ZERO & ZERO & ZERO & \\
\hline $\mathrm{N} 2$ & ZERO & ZERO & ZERO & ZERO & ZERO & ZERO & \\
\hline NOx & ZERO & ŻERO & ZERO & ZERO & ZERO & ZERO & \\
\hline $\mathrm{H} 2 \mathrm{O}$ & $1.190 \mathrm{E}+06$ & 1.452E+06 & ZERO & 1.452E+06 & $2.647 \mathrm{E}+05$ & 1.187E+06 & \\
\hline Nitric Acid (M) & ZEAO & $1.560 \mathrm{E}-01$ & ZERO & $1.717 \mathrm{E}-01$ & $1.107 \mathrm{E}-01$ & $1.717 \mathrm{E}-01$ & \\
\hline $\begin{array}{l}\text { Oxalic Acid (M) } \\
M n+2 \text { (M) }\end{array}$ & $1.000 \mathrm{E} \cdot 01$ & $1.263 \mathrm{E}-01$ & ZERO & $1.389 E \cdot 01$ & 8.962E-02 & 1.389E-01 & \\
\hline$\frac{M n+2(M)}{\text { Heat }(W)}$ & & $6.084 \mathrm{E}+03$ & $6.082 \mathrm{E}+03$ & $2.364 E+00$ & $6.082 E+03$ & $1.933 E+00$ & \\
\hline$Q(H 2)$ & & $1.074 \mathrm{E}+00$ & & $1.062 E+\infty 0$ & $1.112 E+\infty 0$ & $1.062 E+00$ & \\
\hline $\mathrm{H} 2$ (g-mola/hr) & & $2.439 \mathrm{E}+00$ & & $9.365 \mathrm{E}-04$ & $2,524 E+00$ & $7.658 \mathrm{E}-04$ & \\
\hline Air Purge (SCFM) & & $3.107 E+00$ & & $1.193 \mathrm{E} .03$ & $3.215 \mathrm{E}+00$ & $9.755 \mathrm{E}-04$ & \\
\hline Volume (l) & $1.197 \mathrm{E}+03$ & $1.611 \mathrm{E}+03$ & $1.468 E+02$ & $1.464 \mathrm{E}+03$ & $4.138 \mathrm{E}+02$ & $1.197 \mathrm{E}+03$ & \\
\hline Mass (kg) & $1.201 E+03$ & $1.654 E+03$ & $1.654 E+02$ & $1.489 E+03$ & $4.368 E+02$ & $1.217 \mathrm{E}+03$ & \\
\hline Solids (g) & ZERO & 1.680E+05 & $1.654 E+05$ & $2.630 E+03$ & $1.658 E+05$ & $2.151 E+03$ & \\
\hline Solids (g/) & ZERo & $1.043 E+02$ & $1.126 E+03$ & $1.797 \mathrm{E}+00$ & $4.008 E+02$ & $1.797 \mathrm{E}+00$ & \\
\hline Density (g/ml) & 16038600 & $1.027 E+00$ & $1.126 \mathrm{E}+00$ & $1.017 \mathrm{E}+00$ & $1.056 E+00$ & $1.017 E+00$ & \\
\hline Phass & Liguíd & Liquida & Solld & Liquid & Lquild & Liquid & \\
\hline
\end{tabular}


WSRC-TR-2000-00218

Revision 1

Page 18 of 42

Am/Cm Material Balance Spreadsheet Page 21

Version 2

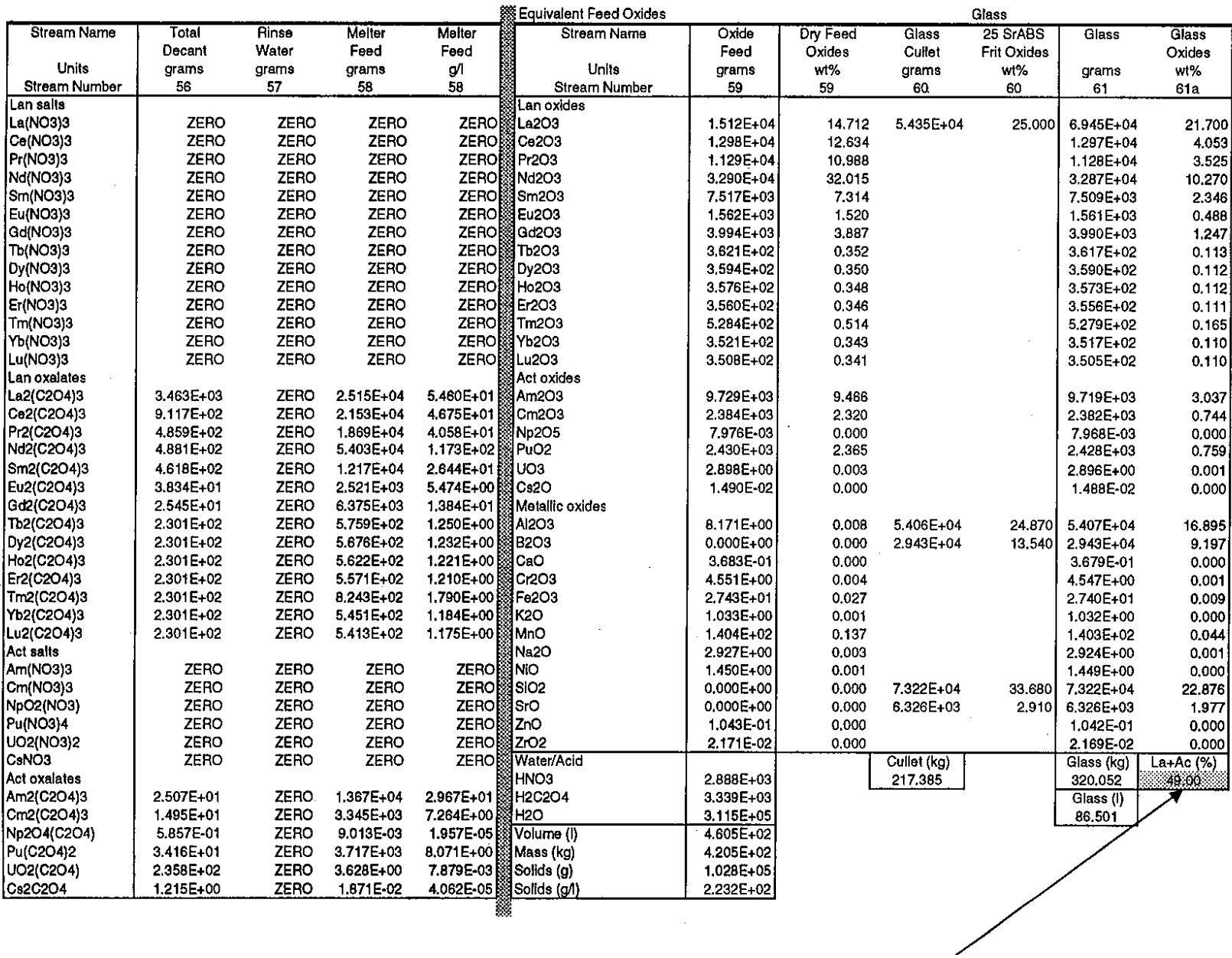


WSRC-TR-2000-00218

Revision 1

Page 19 of 42

Am/Cm Material Balance Spreadsheet Page 22

Version 2

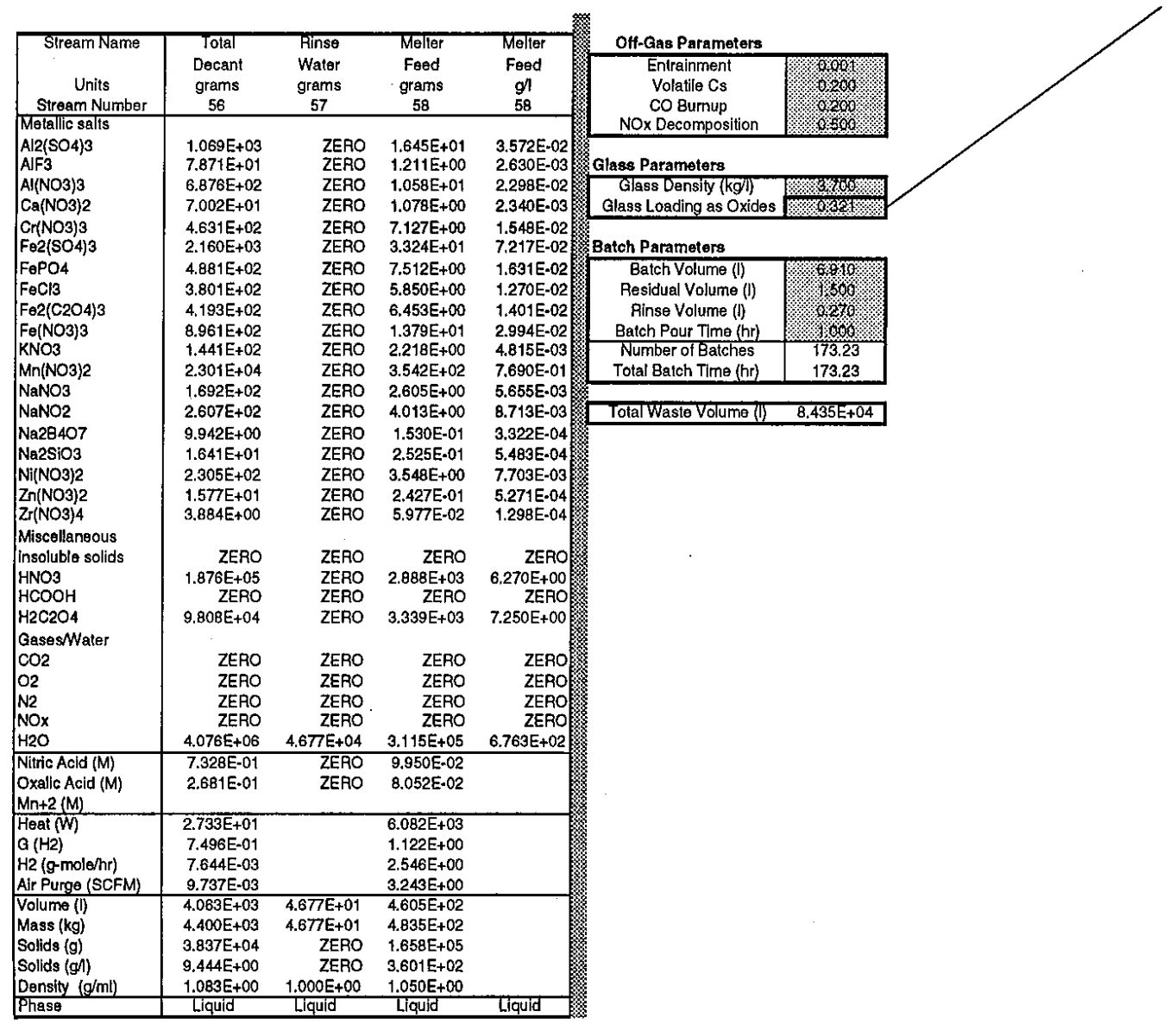


WSRC-TR-2000-00218

Revision 1

Page 20 of 42

Am/Cm Material Balance Spreadsheet Page 23

Version 2

\begin{tabular}{|c|c|c|c|c|c|c|c|c|}
\hline \multicolumn{2}{|l|}{ Melter Off-Gas } & \multicolumn{7}{|l|}{ Off-Gas Flow } \\
\hline $\begin{array}{c}\text { Stream Name } \\
\text { Units } \\
\text { Stream Number }\end{array}$ & $\begin{array}{c}\text { Oxide } \\
\text { Entrainment } \\
\text { Grams } \\
62\end{array}$ & $\begin{array}{c}\text { Stream Name } \\
\text { Units } \\
\text { Stream Number }\end{array}$ & $\begin{array}{c}\text { Sweep Air } \\
\qquad \begin{array}{c}\text { g/hr } \\
63\end{array}\end{array}$ & $\begin{array}{c}\text { Combined } \\
\text { Off-Gas } \\
g / h r \\
64\end{array}$ & $\begin{array}{c}\text { Dilulion Air } \\
\qquad \begin{array}{c}9 / \mathrm{hr} \\
65\end{array}\end{array}$ & $\begin{array}{c}\text { Combined } \\
\text { Off-Gas } \\
\text { g/hr } \\
66\end{array}$ & $\begin{array}{c}\text { Motive Air } \\
\text { g/hr } \\
67\end{array}$ & $\begin{array}{c}\text { Combined } \\
\text { Oft-Gas } \\
\text { g/hr } \\
68\end{array}$ \\
\hline Lan oxides & & \begin{tabular}{|l} 
Lan oxides \\
\end{tabular} & & & & & & \\
\hline $\mathrm{La} 2 \mathrm{O} 3$ & $1.512 \mathrm{E}+01$ & La2O3 & ZERO & $8.728 \mathrm{E}-02$ & ZERO & $8.728 \mathrm{E}-02$ & ZERO & B.728E-02 \\
\hline $\mathrm{Ce} 2 \mathrm{O} 3$ & $1.298 \mathrm{E}+01$ & $\mathrm{C} 82 \mathrm{O}$ & ZERO & $7.495 \mathrm{E}-02$ & ZERO & $7.495 \mathrm{E}-02$ & ZERO & $7.495 \mathrm{E}-02$ \\
\hline Pr2O3 & $1.129 E+01$ & Pr2O3 & ZERO & $6.519 \mathrm{E}-02$ & ZERO & $6.519 \mathrm{E}-02$ & ZERO & $6.519 \mathrm{E}-02$ \\
\hline Nd2O3 & $3.290 \mathrm{E}+01$ & $\mathrm{Nd2O3}$ & ZERO & $1.899 \mathrm{E}-01$ & ZERO & 1.899E-01 & ZERO & $1.899 \mathrm{E}-01$ \\
\hline Sm203 & $7.517 E+00$ & $\mathrm{Sm} 203$ & ZERO & 4.339E-02 & ZERO & 4.339E-02 & ZERO & 4.339E-02 \\
\hline Eu2O3 & $1.562 E+00$ & Eu2O3 & ZERO & $9.018 \mathrm{E} \cdot 03$ & ZERO & $9.018 \mathrm{E}-03$ & ZERO & $9.018 \mathrm{E}-03$ \\
\hline Gd2O3 & $3.994 \mathrm{E}+00$ & Gd2O3 & ZERO & 2,306E-02 & ZERO & $2.306 \mathrm{E}-02$ & ZERO & $2.306 \mathrm{E}-02$ \\
\hline Tb2O3 & $3.621 \mathrm{E}-01$ & $\mathrm{~Tb} 2 \mathrm{O} 3$ & ZERO & $2.090 \mathrm{E}=03$ & ZERO & $2.090 \mathrm{E}-03$ & ZERO & $2.090 \varepsilon .03$ \\
\hline Dy203 & $3.594 \mathrm{E}-01$ & Dy203 & ZERO & $2.075 E-03$ & ZERO & 2.075E-03 & ZERO & $2.075 \mathrm{E}-03$ \\
\hline $\mathrm{Ho} 2 \mathrm{O} 3$ & $3.576 \mathrm{E}-01$ & $\mathrm{Ho2O3}$ & ZERO & $2.065 \mathrm{E}-03$ & ZERO & $2.065 \mathrm{E} \cdot 03$ & ZERO & $2.065 \mathrm{E}-03$ \\
\hline Er2O3 & $3.560 \mathrm{E}-01$ & Er2O3 & ZERO & $2.055 \mathrm{E}-03$ & ZERO & $2.055 \mathrm{E}-03$ & ZERO & $2.055 \mathrm{E}-03$ \\
\hline $\operatorname{Tm} 203$ & $5,284 E-01$ & Tm203 & ZERO & $3.051 \mathrm{E}-03$ & ZERO & $3.051 \mathrm{E}-03$ & ZERO & $3.051 \mathrm{E}-03$ \\
\hline Yb2O3 & 3.521E-01 & Yb2O3 & ZERO & $2.032 \mathrm{E} \cdot 03$ & ZERO & 2.032E-03 & ZERO & 2.032E-03 \\
\hline Lu2O3 & $3.508 \mathrm{E}-01$ & Lu2O3 & ZERO & $2.025 \mathrm{E}-03$ & ZERO & $2.025 \mathrm{E}-03$ & ZERO & $2.025 \mathrm{E}-03$ \\
\hline Act oxides & & Act oxides & & & & & & \\
\hline Am2O3 & $9.729 E+00$ & Am2O3 & ZERO & $5.616 \mathrm{E}-02$ & ZERO & $5.616 \mathrm{E}-02$ & ZERO & $5.616 \mathrm{E}-02$ \\
\hline $\mathrm{Cm} 2 \mathrm{O} 3$ & $2.384 E+00$ & CmeO3 & ZERO & $1.376 \mathrm{E}-02$ & ZERO & $1.376 \mathrm{E}-02$ & ZERO & 1,376E-02 \\
\hline $\mathrm{Np2O5}$ & $7.976 \mathrm{E}-06$ & Np2O5 & ZERO & $4.604 \mathrm{E}-08$ & ZERO & 4.604E-08 & ZERO & 4.604E-08 \\
\hline PuO2 & $2.430 E+00$ & PuO2 & ZERO & $1.403 \mathrm{E}-02$ & ZERO & 1.403E-02 & ZERO & $1.403 \mathrm{E}-02$ \\
\hline vo3 & $2.898 \mathrm{E}-03$ & |UO3 & ZERO & $1.673 \mathrm{E}-05$ & ZERO & $1.673 \mathrm{E}-05$ & ZERO & 1.673E-05 \\
\hline Cs2O & $1.490 \mathrm{E}-05$ & $\mathrm{Cs} 2 \mathrm{O}$ & ZERO & $8.601 E-08$ & ZERO & $8.601 E-08$ & ZERO & $8.601 \mathrm{E}-08$ \\
\hline Metallic oxides & & Metallic oxides & & & & & & \\
\hline Al2O3 & 8.171E-03 & $\mathrm{Al} 2 \mathrm{O} 3$ & ZERO & 4.717E-05 & ZERO & 4.717E-05 & ZERO & $4.717 \mathrm{E}-05$ \\
\hline $\mathrm{B} 2 \mathrm{O} 3$ & $0.000 \mathrm{E}+00$ & 8203 & ZERO & $0.000 E+00$ & ZERO & $0.000 E+00$ & ZERO & $0.000 \mathrm{E}+00$ \\
\hline $\mathrm{CaO}$ & $3.683 E-04$ & $\mathrm{CaO}$ & ZERO & $2.126 \mathrm{E}-06$ & ZERO & $2.126 \mathrm{E}-06$ & ZERO & $2,126 \mathrm{E} \cdot 06$ \\
\hline $\mathrm{Cr} 2 \mathrm{O} 3$ & 4.551E-03 & $\mathrm{Cr} 2 \mathrm{O} 3$ & ZERO & $2.627 \mathrm{E}-0.5$ & ZERO & 2.627E-05 & ZERO & 2.627E-05 \\
\hline $\mathrm{Fe}_{2} \mathrm{O} 3$ & $2.743 \mathrm{E}-02$ & $\mathrm{Fe} 2 \mathrm{O3}$ & ZERO & $1.583 \mathrm{E}-04$ & ZERO & $1.583 \mathrm{E}-04$ & ZERO & $1.583 \mathrm{E}-04$ \\
\hline K2O & $1.033 \mathrm{E}-03$ & $\mathrm{~K} 2 \mathrm{O}$ & ZERO & $5.964 \mathrm{E} \cdot 06$ & ZERO & $5.964 \mathrm{E}-06$ & ZERO & 5.964E-06 \\
\hline Mno & $1.404 \mathrm{E}-01$ & MnO & ZERO & B.104E-04 & ZERO & 8.104E-04 & ZERO & $8.104 \mathrm{E}-04$ \\
\hline $\mathrm{Na} 2 \mathrm{O}$ & $2.927 \mathrm{E}-03$ & $\mathrm{Na} 2 \mathrm{O}$ & ZERO & 1.690E-05 & ZERO & $1.690 \mathrm{E}-05$ & ZERO & $1.690 \mathrm{E}-05$ \\
\hline $\mathrm{NiO}$ & $1.450 \mathrm{E}-03$ & $\mathrm{NiO}$ & ZERO & 8.373E-06 & ZERO & $8.373 \mathrm{E} \cdot 06$ & ZERO & $8.373 \mathrm{E}-06$ \\
\hline SiO2 & $0.000 \mathrm{E}+\infty$ & $\mathrm{SiO} 2$ & ZERO & $0.000 E+00$ & ZERO & $0.000 E+00$ & ZERO & $0.000 E+00$ \\
\hline Sro & $0.000 \mathrm{E}+00$ & Sro & ZERO & $0.000 \mathrm{E}+00$ & ZERO & $0.000 \mathrm{E}+00$ & ZERO & $0.000 \mathrm{E}+00$ \\
\hline Zno & $1.043 \mathrm{E}-04$ & $2 n O$ & ZERO & $6.021 \mathrm{E} \cdot 07$ & ZERO & $6.021 \mathrm{E}-07$ & ZERO & $6.021 \mathrm{E}-07$ \\
\hline 202 & $2.171 \mathrm{E}-05$ & $\mathrm{ZrO} 2$ & ZERO & $1,253 \mathrm{E} \cdot 07$ & ZERO & 1.253E-07 & ZERO & $1.253 \mathrm{E}-07$ \\
\hline Total Mass (kg) & $1.028 \mathrm{E}-01$ & & & & & & & \\
\hline
\end{tabular}


WSRC-TR-2000-00218

Revision 1

\begin{tabular}{|c|c|c|c|c|c|c|c|c|}
\hline $\begin{array}{c}\text { Aif Purge } \\
\text { to Tanks } \\
\text { Units } \\
\text { Siream Number } \\
\end{array}$ & $\begin{array}{l}\text { Tank } \\
17.1 \\
\text { g/hr } \\
83 \\
\end{array}$ & $\begin{array}{c}\text { Evap } \\
16.1 \mathrm{E} \\
\text { g/hr } \\
84 \\
\end{array}$ & $\begin{array}{l}\text { Tank } \\
16.3 \\
\text { g/hr } \\
\text { B5 } \\
\end{array}$ & $\begin{array}{l}\text { Evap } \\
17.3 \\
9 / \mathrm{hr} \\
86 \\
\end{array}$ & $\begin{array}{l}\text { Feed } \\
\text { Tank } \\
\text { g/hr } \\
87 \\
\end{array}$ & $\begin{array}{l}\text { Batch } \\
\text { Tank } \\
\text { g/hr } \\
88\end{array}$ & $\begin{array}{l}\text { Precip } \\
\text { Tank } \\
\text { g/hr } \\
89\end{array}$ & $\begin{array}{c}\text { Feed Prep } \\
\text { Vessel Vent } \\
\text { g/hr } \\
90\end{array}$ \\
\hline Gases Water Vapor & & & & & & & & \\
\hline $\begin{array}{l}\mathrm{CO} 2 \\
\mathrm{O} 2\end{array}$ & $1.310 E+03$ & $1.257 \mathrm{E}+03$ & $6.857 E+00$ & $1.257 E+03$ & $3.883 E+01$ & $5.590 \mathrm{E}+00$ & $2.723 E+0 t$ & $3.903 E+03$ \\
\hline $\begin{array}{l}\text { N2 } \\
\text { NOx }\end{array}$ & $4.212 \mathrm{E}+03$ & $4.043 E+03$ & $2.205 E+01$ & $4.043 \mathrm{E}+03$ & $1.249 \mathrm{E}+02$ & 1.797E+01 & $8.757 E+01$ & 1.255E+04 \\
\hline $\mathrm{H} 2 \mathrm{O}$ & $8.069 \mathrm{E}+01$ & $7.745 E+01$ & 4.223E-01 & $7.745 \mathrm{E}+01$ & $2.392 E+00$ & 3.443E-01 & $1.677 E+00$ & $2.404 E+02$ \\
\hline Tank Volume (I) & & & & & 48.00 & 6.91 & 6.91 & \\
\hline Purge Flow (SCFM) & $2.583 E+00$ & $2.479 \mathrm{E}+00$ & 1.352E-02 & $2.479 E+00$ & $7.655 \mathrm{E}-02$ & $1.102 \mathrm{E}-02$ & $5,369 \mathrm{E}-02$ & $7.696 \mathrm{E}+00$ \\
\hline Temperature (C) & 20.00 & 20.00 & 20.00 & 20.00 & 20.00 & 20.00 & 20.00 & 20.00 \\
\hline $\begin{array}{l}\text { Temperature (K) } \\
\text { Psat (atm) }\end{array}$ & $\begin{array}{r}2.932 E+02 \\
2.288 E-02\end{array}$ & $\begin{array}{r}2.932 E+02 \\
2.28 B E-02\end{array}$ & $\begin{array}{l}2.932 E+02 \\
2.288 E-02\end{array}$ & $\begin{array}{r}2.932 E+02 \\
2.288 E-02\end{array}$ & $\begin{array}{r}2.932 \mathrm{E}+02 \\
2.288 \mathrm{E}-02\end{array}$ & $\begin{array}{r}2.932 E+02 \\
2.288 E-02\end{array}$ & $\begin{array}{r}2.932 E+02 \\
2.288 E-02\end{array}$ & $\begin{array}{r}2.932 E+02 \\
2.288 E-02\end{array}$ \\
\hline Volume Flow $(\mathrm{Vhr})$ & $4.710 E+03$ & $4.520 \mathrm{E}+03$ & $2.465 E+01$ & $4.520 E+03$ & $1.396 E+02$ & $2,010 E+01$ & $9.790 \mathrm{E}+01$ & $1.403 E+04$ \\
\hline
\end{tabular}

\begin{tabular}{|c|c|c|c|}
\hline $\begin{array}{l}\text { Melter } \\
\text { Mass Check } \\
\text { Input }\end{array}$ & $\mathrm{kg}$ & Output & $\mathrm{kg}$ \\
\hline $\begin{array}{c}\text { Feed Material } \\
\text { Glass Cullet }\end{array}$ & $\begin{array}{l}483.544 \\
217.385\end{array}$ & $\begin{array}{r}\text { Glass } \\
\text { Entrainment }\end{array}$ & $\begin{array}{r}920.052 \\
0.103\end{array}$ \\
\hline & & $\begin{array}{r}\text { Water } \\
\text { Calcine Rxn Prod. } \\
\text { Vitrify Rxn Prod. }\end{array}$ & $\begin{array}{r}311.475 \\
68.666 \\
0.633\end{array}$ \\
\hline TotalMass & 700.929 & Total Mass & 700.929 \\
\hline
\end{tabular}


WSRC-TR-2000-00218

Revision 1

Page 22 of 42

Am/Cm Material Balance Spreadsheet Page 25

Version 2

\begin{tabular}{|c|c|}
\hline Melter Olf-Gas & Drying \\
\hline $\begin{array}{c}\text { Stream Name } \\
\text { Units } \\
\text { Stream Number }\end{array}$ & $\begin{array}{c}\text { Reaction } \\
\text { Products } \\
\text { grams } \\
62\end{array}$ \\
\hline $\begin{array}{l}\text { Gases } \\
\mathrm{H} 2 \mathrm{O} \\
\mathrm{CO} \\
\mathrm{CO} 2 \\
\mathrm{O} 2 \\
\mathrm{~N} 2 \\
\mathrm{NO} \times(\mathrm{NO}+\mathrm{NO} 2) / 2 \\
\mathrm{SO} 3 \\
\mathrm{PO} \times(\mathrm{PO} 2+\mathrm{PO}) / 2 \\
\mathrm{HCl} \\
\mathrm{HF}\end{array}$ & 3.115E+05 \\
\hline $\begin{array}{l}\text { Temperature (C) } \\
\text { Pressure (in. H2O) } \\
\text { Volume (l) } \\
\text { Mass }(g) \\
\text { Solids (g) } \\
\text { Solids (g/) } \\
\text { Density (g/) } \\
\text { Phase }\end{array}$ & $\begin{array}{l}\% \\
6.745 E+05 \\
3.115 E+05 \\
1.028 E+02 \\
1.524 E-04 \\
4.619 E-01 \\
\text { Vapor }\end{array}$ \\
\hline
\end{tabular}

\begin{tabular}{|c|c|c|c|c|c|c|}
\hline $\begin{array}{c}\text { Stream Name } \\
\text { Units } \\
\text { Stream Number }\end{array}$ & $\begin{array}{c}\text { Sweep Air } \\
\text { g/hr } \\
63\end{array}$ & $\begin{array}{c}\text { Combined } \\
\text { Off-Gas } \\
g / h t \\
64\end{array}$ & $\begin{array}{l}\text { Dilution Air } \\
\qquad \begin{array}{c}g / h r \\
65\end{array}\end{array}$ & $\begin{array}{l}\text { Combined } \\
\text { Off-Gas } \\
g^{\prime / h r} \\
66\end{array}$ & $\begin{array}{c}\text { Motive Air } \\
\text { g/hr } \\
67\end{array}$ & $\begin{array}{c}\text { Combined } \\
\text { Off-Gas } \\
\text { g/hr } \\
68\end{array}$ \\
\hline ases & & & & & & \\
\hline $\begin{array}{l}\mathrm{H} 2 \mathrm{O} \\
\mathrm{CO} \\
\mathrm{CO} 2\end{array}$ & $2.031 E+03$ & $3.829 \mathrm{E}+03$ & $1.502 \mathrm{E}+02$ & $3.979 E+03$ & $2.497 \mathrm{E}+02$ & $4.229 \mathrm{E}+03$ \\
\hline $\mathrm{O} 2$ & $3.297 \mathrm{E}+04$ & $3.297 E+04$ & $4.592 E+04$ & $7.889 E+04$ & $7.633 E+04$ & $1.552 E+05$ \\
\hline $\begin{array}{l}\mathrm{N} 2 \\
\mathrm{NO}(\mathrm{NO}+\mathrm{NO} 2) / 2 \\
\text { SO3 } \\
\mathrm{PO}(\mathrm{PO} 2+\mathrm{PO} 3) / 2 \\
\mathrm{HCl} \\
\mathrm{HF}\end{array}$ & $1.060 \mathrm{E}+05$ & $1.060 \mathrm{E}+05$ & $1.687 \mathrm{E}+05$ & $2.747 E+05$ & $2.804 \mathrm{E}+05$ & $5.550 E+05$ \\
\hline
\end{tabular}

\begin{tabular}{|c|c|c|c|c|c|c|}
\hline Temperature (C) & 20 & 24.26 & 20.00 & 21.70 & 20.00 & 20.85 \\
\hline Pressure (in, $\mathrm{H} 2 \mathrm{O}$ ) & $66,6 \mathrm{e}$ & 0.00 & 0.00 & 0.00 & 0.00 & 0.00 \\
\hline Volume Flow (hr) & $1.185 E+05$ & $1.227 \mathrm{E}+0.5$ & $1.790 E+05$ & $3.022 \mathrm{E}+05$ & $2.975 E+05$ & $6.006 \mathrm{E}+05$ \\
\hline Mass Flow (ghr) & $1.410 E+05$ & $1.428 E+05$ & $2.146 E+05$ & $3.575 E+05$ & $3.567 E+05$ & $7.145 E+05$ \\
\hline Solids Flow $(g / h r)$ & & 5.933E-01 & & 5.933E-01 & & $5,933 \mathrm{E}-01$ \\
\hline Solids $\left(g^{\prime}\right)$ & & $4.836 \mathrm{E} \cdot 06$ & & $1.963 E-06$ & & $9.877 \mathrm{E}-07$ \\
\hline Density (g/) & $1.190 \mathrm{E}+00$ & $1.164 \mathrm{E}+\infty 0$ & $1.199 E+00$ & $1.183 E+\infty$ & $1.199 E+00$ & $1.190 E+00$ \\
\hline Phase & Vapor & Vapor & Vapor & Vapor & Vapor & Vapor \\
\hline Flow (SCFM) & 6500 & $6.633 \mathrm{E}+01$ & 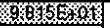 & $1.648 E+02$ & $1.632 E+02$ & $3.285 E+02$ \\
\hline Temperature (K) & $2.932 \mathrm{E}+02$ & $2.974 \mathrm{E}+02$ & $2.932 \mathrm{E}+02$ & $2.949 E+02$ & $2.932 E+02$ & $2.940 \mathrm{E}+02$ \\
\hline Molecular Weight & 2.861E+01 & $2.840 E+01$ & $2.884 E+01$ & $2.866 E+01$ & $2.884 E+01$ & $2.875 E+01$ \\
\hline Pvap (atm) & 2.289E-02 & $4.242 \mathrm{E}-02$ & $1.125 \mathrm{E}-03$ & $1.777 \mathrm{E}-02$ & $1.125 \mathrm{E}-03$ & $9.483 \mathrm{E} \cdot 03$ \\
\hline Psat (atm) & $2.288 \mathrm{E}-02$ & $2.963 \mathrm{E}-02$ & $2.288 \mathrm{E}-02$ & 2.539E-02 & $2.288 \mathrm{E}-02$ & $2.411 \mathrm{E}-02$ \\
\hline Dow Point (C) & $1.992 E+01$ & $3.027 E+01$ & $-2.080 \mathrm{E}+01$ & $1.592 E+01$ & $-2.080 E+01$ & $6.488 E+00$ \\
\hline Humidity $(\%)$ & 40 & $1.432 E+02$ & $4.918 E+0.0 ?$ & $000 \%$ & $4.918 E+00$ & $3.934 E+01$ \\
\hline$\Delta \mathrm{T}(\mathrm{C})$ & & $-6,015 E+00$ & & $5.781 E+00$ & & $1.436 \mathrm{E}+01$ \\
\hline
\end{tabular}


WSRC-TR-2000-00218

Revision 1

Page 23 of 42

Am/Cm Material Balance Spreadsheet Page 26

Version 2

\begin{tabular}{|c|c|}
\hline Aelter Oft-Gas & Drying \\
\hline $\begin{array}{c}\text { Stream Name } \\
\text { Units } \\
\text { Stream Number }\end{array}$ & $\begin{array}{l}\text { Reaction } \\
\text { Products } \\
\text { grams } \\
62\end{array}$ \\
\hline Gases & \\
\hline $\begin{array}{l}\mathrm{H} 2 \mathrm{O} \\
\mathrm{CO} \\
\mathrm{CO} 2 \\
\mathrm{O} 2 \\
\mathrm{~N} 2 \\
\mathrm{NO} \times(\mathrm{NO}+\mathrm{NO} 2) / 2 \\
\mathrm{SO} 3 \\
\mathrm{PO} \times(\mathrm{PO} 2+\mathrm{PO} 3) / 2 \\
\mathrm{HO} \\
\mathrm{HF}\end{array}$ & $3.115 E+05$ \\
\hline $\begin{array}{l}\text { Temperature (C) } \\
\text { Pressure (in. H2O) } \\
\text { Volume (l) } \\
\text { Mass (g) } \\
\text { Solids (g) } \\
\text { Solids (g/) } \\
\text { Density (g/) } \\
\text { Phase }\end{array}$ & $\begin{array}{l}.2000 \\
6.745 \mathrm{E}+05 \\
3.115 \mathrm{E}+05 \\
1.028 \mathrm{E}+02 \\
1.524 \mathrm{E}-04 \\
4.619 \mathrm{E}-01 \\
\text { Vapor }\end{array}$ \\
\hline Phase & Vapor \\
\hline
\end{tabular}

\begin{tabular}{|c|c|c|c|c|c|c|}
\hline $\begin{array}{c}\text { Stream Name } \\
\text { Units } \\
\text { Stream Number }\end{array}$ & $\begin{array}{l}\text { Sweep Alr } \\
\text { g/hr } \\
63\end{array}$ & $\begin{array}{c}\text { Combined } \\
\text { Off-Gas } \\
9 / \mathrm{hr} \\
64 \\
\end{array}$ & $\begin{array}{l}\text { Dilution Air } \\
\qquad \begin{array}{l}9 / \mathrm{hr} \\
65\end{array}\end{array}$ & $\begin{array}{c}\text { Combined } \\
\text { Off-Gas } \\
\text { g/hr } \\
66\end{array}$ & $\begin{array}{l}\text { Molive Ail } \\
\text { g/hr } \\
67\end{array}$ & $\begin{array}{c}\text { Combined } \\
\text { Off-Gas } \\
9 \mathrm{hr} \\
68\end{array}$ \\
\hline Gases & & & & & & \\
\hline $\begin{array}{l}\mathrm{H} 2 \mathrm{O} \\
\mathrm{CO} \\
\mathrm{CO} 2 \\
\mathrm{O} 2\end{array}$ & $2.031 E+03$ & $3.829 \mathrm{E}+03$ & & $3.829 \mathrm{E}+03$ & $9.947 E+01$ & $3.928 \mathrm{E}+03$ \\
\hline $\begin{array}{l}\mathrm{O} 2 \\
\mathrm{~N} 2 \\
\mathrm{NOx}(\mathrm{NO}+\mathrm{NO} 2) / 2 \\
\mathrm{SO} 3 \\
\mathrm{POx}(\mathrm{PO} 2+\mathrm{PO} 3) / 2 \\
\mathrm{HCl} \\
\mathrm{HF}\end{array}$ & $\begin{array}{l}3.297 \mathrm{E}+04 \\
1.060 \mathrm{E}+05\end{array}$ & $\begin{array}{l}3.297 E+04 \\
1.060 E+05\end{array}$ & & $\left.\begin{array}{l}3.297 \mathrm{E}+04 \\
1.060 \mathrm{E}+05\end{array}\right]$ & $\begin{array}{l}3.041 E+04 \\
1.117 E+05\end{array}$ & $\begin{array}{l}6.338 E+04 \\
2.177 E+05\end{array}$ \\
\hline Temperature $(\mathrm{C})$ & $20 \% 0$ & 24.26 & & 363 & 20,00 & 28.39 \\
\hline Pressure (in. $\mathrm{H} 2 \mathrm{O}$ ) & \%. & 0.00 & & 0.00 & 0.00 & 0.00 \\
\hline Volume Fiow (Vhr) & $1.185 E+05$ & $1.227 E+05$ & & $1.278 \mathrm{E}+05$ & $1.185 E+05$ & $2.467 E+05$ \\
\hline Mass Flow (g/hr) & $1.410 E+05$ & $1.428 E+05$ & & $1.428 \mathrm{E}+05$ & $1.421 E+05$ & $2.850 \mathrm{E}+05$ \\
\hline Solids Flow (g/hr) & & $5.933 E-01$ & & $5.933 \mathrm{E}-01$ & & $5.933 E-01$ \\
\hline Solids $(g /)$ & & $4.836 \mathrm{E}-06$ & & $4.641 \mathrm{E}-06$ & & $2.405 \mathrm{E}-06$ \\
\hline Density $(g /)$ & $1.190 \mathrm{E}+00$ & $1.164 E+00$ & & $1.117 E+00$ & $1.199 E+00$ & $1.155 E+00$ \\
\hline Phase & Vapor & Vapor & Vapor & Vapor & Vapot & Vapor \\
\hline Flow (SCFM) & 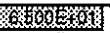 & $6.633 E+01$ & & $6.633 \mathrm{E}+01$ & $6.500 \mathrm{E}+01$ & $1.315 \mathrm{E}+02$ \\
\hline $\begin{array}{l}\text { Temperature (K) } \\
\text { Molecular Welght }\end{array}$ & $\begin{array}{l}2.932 \mathrm{E}+02 \\
2.861 \mathrm{E}+01\end{array}$ & $\begin{array}{l}2.974 \mathrm{E}+02 \\
2.840 \mathrm{E}+01\end{array}$ & & $\begin{array}{l}3.099 \mathrm{E}+02 \\
2.840 \mathrm{E}+01\end{array}$ & $\begin{array}{l}2.932 E+02 \\
2.884 E+01\end{array}$ & $\begin{array}{l}3.015 E+02 \\
2.862 E+01\end{array}$ \\
\hline Pvap (atm) & 2.288E-02 & 4.242E-02 & & $4.242 \mathrm{E}-02$ & 1.125E-03 & $2.198 \mathrm{E}-02$ \\
\hline Psat (atm) & 2.28日E-02 & 2.963E-02 & & $6.060 \mathrm{E}-02$ & $2.288 E-02$ & $3.781 \mathrm{E}-02$ \\
\hline Dew Point $(C)$ & $1.992 E+0 \dagger$ & $3.027 E+01$ & & $3.027 E+01$ & $-2.080 E+01$ & $1.928 E+01$ \\
\hline Humidity $(\%)$ & 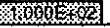 & $1.432 E+02$ & & \%o@ext & $4.918 \mathrm{E}+00$ & $5.812 E+01$ \\
\hline$\overline{\Delta T}(\mathrm{C})$ & & $-6.015 \mathrm{E}+00$ & & $6.461 \mathrm{E}+00$ & & $9.111 E+00$ \\
\hline
\end{tabular}


WSRC-TR-2000-00218

Revision 1

Page 24 of 42

Am/Cm Material Balance Spreadsheet Page 27

Version 2

\begin{tabular}{|c|c|}
\hline Melter Off-Gas & Calcination \\
\hline $\begin{array}{c}\text { Stream Name } \\
\text { Units } \\
\text { Stream Number }\end{array}$ & $\begin{array}{c}\text { Reaction } \\
\text { Products } \\
\text { grams } \\
69\end{array}$ \\
\hline Gases & \\
\hline $\mathrm{H} 2 \mathrm{O}$ & $1.079 \mathrm{E}+03$ \\
\hline & $2.546 \mathrm{E}+04$ \\
\hline $\mathrm{CO} 2$ & $4.000 E+04$ \\
\hline $\mathrm{O} 2$ & $1.719 E+02$ \\
\hline & \\
\hline $\mathrm{NO} \times(\mathrm{NO}+\mathrm{NO} 2) / 2$ & $1.914 \mathrm{E}+03$ \\
\hline $\mathrm{SO} 3$ & 3.151E+01 \\
\hline $\mathrm{PO} \times(\mathrm{PO} 2+\mathrm{PO} 3) / 2$ & $1.767 E+00$ \\
\hline $\mathrm{HOl}$ & $3.945 E+00$ \\
\hline HF & $8.658 \mathrm{E}-01$ \\
\hline $\begin{array}{l}\text { Temperature (C) } \\
\text { Pressure (in. H2O) }\end{array}$ & 20100 \\
\hline Volume (l) & $1.552 \mathrm{E}+05$ \\
\hline $\begin{array}{l}\text { Mass (g) } \\
\text { Solids (g) }\end{array}$ & $6.867 \mathrm{E}+04$ \\
\hline $\begin{array}{l}\text { Solids (g } \\
\text { Solids (g }\end{array}$ & \\
\hline Density $(g /)$ & 4.424E-01 \\
\hline Phase & Vapor \\
\hline
\end{tabular}

Off-Gas Flow

\begin{tabular}{|c|c|c|c|c|c|c|}
\hline $\begin{array}{c}\text { Stream Name } \\
\text { Units } \\
\text { Stream Number }\end{array}$ & $\begin{array}{c}\text { Sweep Air } \\
\qquad \begin{array}{c}9 / h r \\
70\end{array}\end{array}$ & $\begin{array}{c}\text { Combined } \\
\text { Otf-Gas } \\
g^{\prime h r} \\
71\end{array}$ & $\begin{array}{l}\text { Dilution Air } \\
\qquad \begin{array}{l}\text { g/hr } \\
72\end{array}\end{array}$ & $\begin{array}{c}\text { Combined } \\
\text { Off-Gas } \\
g / h r \\
73\end{array}$ & $\begin{array}{l}\text { Molive Ais } \\
\qquad \begin{array}{c}\text { ghr } \\
74\end{array}\end{array}$ & $\begin{array}{c}\text { Combined } \\
\text { Otf-Gas } \\
g / h r \\
75\end{array}$ \\
\hline Gases & & & & & & \\
\hline $\begin{array}{l}\mathrm{H} 2 \mathrm{O} \\
\mathrm{CO}\end{array}$ & $2.031 \mathrm{E}+03$ & $\begin{array}{l}2.037 \mathrm{E}+03 \\
1.176 \mathrm{E}+02\end{array}$ & $3.345 \mathrm{E}+01$ & $2.070 \mathrm{E}+03$ & $1.329 \mathrm{E}+02$ & $2,203 E+03$ \\
\hline $\mathrm{CO} 2$ & & $2.771 \mathrm{E}+02$ & & $2.771 E+02$ & & $2.771 E+02$ \\
\hline $\mathrm{O} 2$ & $3.297 E+04$ & $3,296 \mathrm{E}+04$ & $1.023 E+04$ & $4.318 \mathrm{E}+04$ & $4.064 E+04$ & $8.382 E+04$ \\
\hline & $1.060 E+05$ & $1.060 E+05$ & $3.756 E+04$ & $1.436 E+05$ & $1.493 E+05$ & $2.928 E+05$ \\
\hline $\mathrm{NO} \times(\mathrm{NO}+\mathrm{NO} 2) / 2$ & & $5.524 \mathrm{E}+00$ & & $5.524 \mathrm{E}+00$ & & $5.524 \mathrm{E}+00$ \\
\hline $\mathrm{SO}^{\circ}$ & & $1.819 \mathrm{E}-01$ & & $1.819 \mathrm{E}-01$ & & $1.819 \mathrm{E}-01$ \\
\hline $\mathrm{PO} \times(\mathrm{PO} 2+\mathrm{PO} 3) / 2$ & & $1.020 \mathrm{E}-02$ & & $1.020 \mathrm{E} \cdot 02$ & & $1.020 \mathrm{E}-02$ \\
\hline $\mathrm{HOI}$ & & $2.277 \mathrm{E}-02$ & & 2.277E-02 & & $2.277 \mathrm{E}-02$ \\
\hline $\mathrm{HF}$ & & $4.998 \mathrm{E}-03$ & & $4.998 \mathrm{E}-03$ & & $4.998 \mathrm{E}-03$ \\
\hline
\end{tabular}

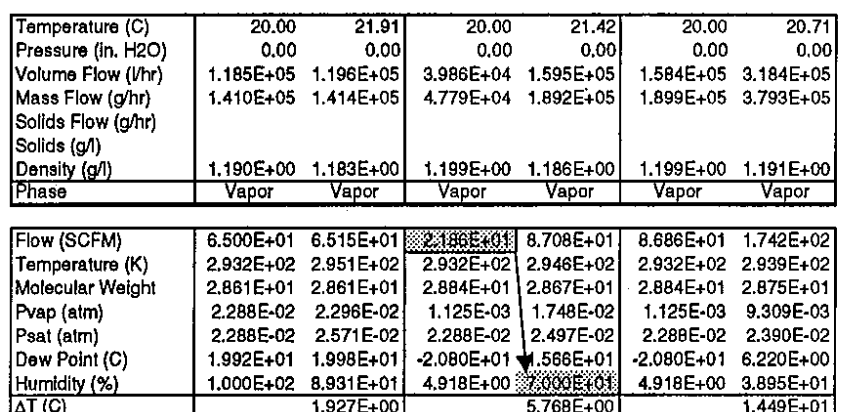

$\Delta \mathrm{T}(\mathrm{C})$ $1.927 \mathrm{E}+00$ $5.768 \mathrm{E}+00$ 
WSRC-TR-2000-00218

Revision 1

Page 25 of 42

Am/Cm Material Balance Spreadsheet Page 28

Version 2

\begin{tabular}{|c|c|}
\hline Melter Off-Gas & Calcination \\
\hline $\begin{array}{c}\text { Stream Name } \\
\text { Units } \\
\text { Stream Number }\end{array}$ & $\begin{array}{c}\text { Poaction } \\
\text { Products } \\
\text { grams } \\
69\end{array}$ \\
\hline Gases & \\
\hline $\begin{array}{l}\mathrm{H} 2 \mathrm{O} \\
\mathrm{CO}\end{array}$ & $\begin{array}{l}1.079 E+03 \\
2.546 E+04\end{array}$ \\
\hline $\mathrm{CO} 2$ & $4.000 \mathrm{E}+04$ \\
\hline $\mathrm{O} 2$ & $1.719 E+02$ \\
\hline $\begin{array}{l}\mathrm{N} 2 \\
\mathrm{NO} \times(\mathrm{NO}+\mathrm{NO} 2) / 2\end{array}$ & $1.914 E+03$ \\
\hline $\mathrm{SO} 3$ & $3.151 E+01$ \\
\hline $\mathrm{PO}(\mathrm{PO} 2+\mathrm{PO} 3) / 2$ & $1.767 \mathrm{E}+00$ \\
\hline $\mathrm{HCl}$ & $3.945 E+00$ \\
\hline $\mathrm{HF}$ & $8.658 \mathrm{E}-01$ \\
\hline $\begin{array}{l}\text { Temperafure (C) } \\
\text { Pressure (in. } \mathrm{H} 2 \mathrm{O} \text { ) }\end{array}$ & 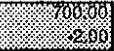 \\
\hline Volume (I) & $1.552 \mathrm{E}+05$ \\
\hline $\begin{array}{l}\text { Mass (g) } \\
\text { Solids (g) }\end{array}$ & $6.867 E+04$ \\
\hline Solids $(g h)$ & \\
\hline Density $(g /)$ & 4.424E-01 \\
\hline Phase & Vapor \\
\hline
\end{tabular}

\begin{tabular}{|c|c|c|c|c|c|c|}
\hline $\begin{array}{c}\text { Stream Name } \\
\text { Units } \\
\text { Stream Number }\end{array}$ & $\begin{array}{c}\text { Sweep Alr } \\
\text { g/hr } \\
70\end{array}$ & $\begin{array}{c}\text { Combined } \\
\text { Off-Gas } \\
\text { g/hr } \\
71\end{array}$ & $\begin{array}{l}\text { Dilution Ali } \\
\qquad \begin{array}{l}\text { ghr } \\
72\end{array}\end{array}$ & $\begin{array}{l}\text { Combined } \\
\text { Off-Gas } \\
\text { g/hr } \\
73\end{array}$ & $\begin{array}{l}\text { Motive Air } \\
\begin{array}{l}0 / \mathrm{hr} \\
74\end{array}\end{array}$ & $\begin{array}{c}\text { Combined } \\
\text { Off-Gas } \\
0 / h r \\
75\end{array}$ \\
\hline Gases & & & & & & \\
\hline $\mathrm{H} 2 \mathrm{O}$ & $2.031 E+03$ & $\begin{array}{l}2.037 \mathrm{E}+03 \\
1.176 \mathrm{E}+02\end{array}$ & & $\begin{array}{l}2.037 \mathrm{E}+03 \\
1.176 \mathrm{E}+02\end{array}$ & $9.947 \mathrm{E}+01$ & $\begin{array}{l}2.136 \mathrm{E}+03 \\
1.176 \mathrm{E}+02\end{array}$ \\
\hline $\mathrm{CO} 2$ & & $2.771 \mathrm{E}+02$ & & $2.771 E+02$ & & $2.771 E+02$ \\
\hline $\mathrm{O} 2$ & $3.297 \mathrm{E}+04$ & $3.296 \mathrm{E}+\mathrm{O}$ & & $3.296 \mathrm{E}+04$ & $3.041 E+04$ & $6.337 \varepsilon+$ \\
\hline $\begin{array}{l}\text { N2 } \\
\text { NOx }(\mathrm{NO}+\mathrm{NO} 2) / 2\end{array}$ & $1.060 E+05$ & $\begin{array}{l}1.060 \mathrm{E}+05 \\
5.524 \mathrm{E}+00\end{array}$ & & $\begin{array}{l}1.060 \mathrm{E}+05 \\
5.524 \mathrm{E}+00\end{array}$ & $1.117 \mathrm{E}+05$ & $\begin{array}{l}2.177 \mathrm{E}+05 \\
5.524 \mathrm{E}+00\end{array}$ \\
\hline $\mathrm{SO} 3$ & & $1.819 \mathrm{E}-01$ & & 1.819E-01 & & $1.819 \mathrm{E} \cdot 0$ \\
\hline $\mathrm{POX}(\mathrm{PO} 2+\mathrm{PO} 3) / 2$ & & $1,020 \mathrm{E}-02$ & & $1.020 \mathrm{E}-02$ & & $1.020 \mathrm{E}$ \\
\hline & & 2,277E-02 & & 2.277E-02 & & $2.277 \mathrm{E} \cdot 02$ \\
\hline $\mathrm{HF}$ & & $4.998 E-03$ & & $4.998 \mathrm{E}-03$ & & $4.998 \mathrm{E} \cdot 0$ \\
\hline
\end{tabular}

\begin{tabular}{|c|c|c|c|c|c|c|}
\hline Temperature (C) & 20.00 & 21.91 & & 2539 & 20.00 & 22.98 \\
\hline Pressure (In. $\mathrm{H} 2 \mathrm{O}$ ) & 0.00 & 0.00 & & 0.00 & 0.00 & 0,00 \\
\hline Volume Flow (/hr) & $1.185 \vec{E}+05$ & $1.196 E+05$ & & $1.212 E+05$ & $1.185 E+05$ & $2.401 E+05$ \\
\hline $\begin{array}{l}\text { Mass Flow }(g / h r) \\
\text { Solids Flow }(g / h r) \\
\text { Solids }(g)\end{array}$ & $1.410 E+05$ & $1.414 E+05$ & & $1.414 E+05$ & $1.421 E+05$ & $2.836 \mathrm{E}+05$ \\
\hline Density (g/1) & $1.190 E+00$ & $1.183 E+\infty$ & & 1.167E+00 & $1.199 \mathrm{E}+00$ & $1.181 E+00$ \\
\hline Phase & Vapor & Vapor & Vapor & \begin{tabular}{|l} 
Vapor \\
\end{tabular} & Vapor & Vapor \\
\hline Flow: & $6.500 E+01$ & $6.515 E+01$ & & $6.515 \mathrm{~F}+01$ & $6.500 \mathrm{E}+01$ & $1304 E+02$ \\
\hline Temper & $2.932 E+02$ & $2.951 \mathrm{E}+02$ & & $2.991 \mathrm{E}+02$ & $2.932 E+02$ & $61 E+02$ \\
\hline Molecular Welght & $2.861 E+01$ & $2.861 E+0$ & & $2.861 E+01$ & $2.884 E+01$ & $2.873 E+01$ \\
\hline Pvap (atm) & 2.28BE-02 & $2.296 \mathrm{E}-02$ & & 2.296E-02 & $1.125 \mathrm{E}-03$ & $1.206 \mathrm{E}-02$ \\
\hline Psat (atm) & $2.288 \mathrm{E}-02$ & 2.571E-02 & & $3.280 \mathrm{E}-02$ & $2.288 \mathrm{E}-02$ & $2.743 \mathrm{E}-02$ \\
\hline Dew Point (C) & $1.992 E+01$ & $1.998 \mathrm{E}+01$ & & $1.998 \mathrm{E}+01$ & $-2,080 E+01$ & $1.001 E+01$ \\
\hline Humidity $(\%)$ & $1.000 \mathrm{E}+02$ & $8.931 E+01$ & & \%orol 20 & $4.918 E+00$ & 4.395E+01 \\
\hline$\Delta \overline{\Delta T}(\mathrm{C})$ & & $1.927 \mathrm{E}+00$ & & $5.986 \mathrm{E}+00$ & & $1.297 \mathrm{E}+01$ \\
\hline
\end{tabular}


WSRC-TR-2000-00218

Revision 1

Page 26 of 42

Am/Cm Material Balance Spreadsheet Page 29

Version 2

\begin{tabular}{|c|c|}
\hline Melter Off-Gas & Vitrification \\
\hline $\begin{array}{c}\text { Stream Name } \\
\text { Units } \\
\text { Stream Number }\end{array}$ & \begin{tabular}{|c|} 
Reaction \\
Products \\
grams \\
76 \\
\end{tabular} \\
\hline \begin{tabular}{|l|} 
Gases \\
$\mathrm{H} 2 \mathrm{O}$ \\
$\mathrm{CO}$ \\
$\mathrm{CO} 2$ \\
$\mathrm{O} 2$ \\
$\mathrm{~N} 2$ \\
$\mathrm{NO} \times(\mathrm{NO}+\mathrm{NO} 2) / 2$ \\
$\mathrm{SO} 3$ \\
$\mathrm{POx}(\mathrm{PO} 2+\mathrm{PO} 3) / 2$ \\
$\mathrm{HCl}$ \\
$\mathrm{HF}$ \\
\end{tabular} & $6.329 E+02$ \\
\hline \begin{tabular}{|l|} 
Temperature (C) \\
Pressure (in. H2O) \\
Volume (l) \\
Mass (g) \\
Solids (g) \\
Solids (g/) \\
Density (gll) \\
\end{tabular} & $\begin{array}{r}0 \% 000 \\
1.587 \mathrm{E}+03 \\
6.329 \mathrm{E}+02\end{array}$ \\
\hline Phase & Vapor \\
\hline
\end{tabular}

\begin{tabular}{|c|c|c|c|c|c|c|}
\hline $\begin{array}{c}\text { Stream Name } \\
\text { Units } \\
\text { Stream Number }\end{array}$ & $\begin{array}{l}\text { Sweep Air } \\
\qquad \begin{array}{c}g / \mathrm{hr} \\
77\end{array}\end{array}$ & $\begin{array}{c}\text { Combined } \\
\text { Off-Gas } \\
g / h r \\
78 \\
\end{array}$ & $\begin{array}{l}\text { Dilution Air } \\
\qquad \begin{array}{l}\text { ghr } \\
79\end{array}\end{array}$ & $\begin{array}{c}\text { Combined } \\
\text { Off-Gas } \\
g / h r \\
80\end{array}$ & $\begin{array}{c}\text { Motive Air } \\
\begin{array}{c}\text { g/hr } \\
81\end{array}\end{array}$ & $\begin{array}{c}\text { Combined } \\
\text { Off-Gas } \\
\text { g/hr } \\
82\end{array}$ \\
\hline $\begin{array}{l}\text { Gases } \\
\mathrm{H} 2 \mathrm{O} \\
\mathrm{CO} \\
\mathrm{CO} 2\end{array}$ & $2.031 E+03$ & $2.031 E+03$ & $4.626 E+01$ & $2.077 E+03$ & $1.457 E+02$ & $2.223 E+03$ \\
\hline $\begin{array}{l}\mathrm{O} 2 \\
\mathrm{~N} 2 \\
\mathrm{NO}(\mathrm{NO}+\mathrm{NO} 2) / 2 \\
\text { SO3 } \\
\mathrm{PO}(\mathrm{PO} 2+\mathrm{PO} 3) / 2 \\
\mathrm{HCl} \\
\mathrm{HF}\end{array}$ & $\begin{array}{l}3.297 E+04 \\
1.060 E+05\end{array}$ & $\begin{array}{l}3.297 \mathrm{E}+04 \\
1.060 \mathrm{E}+05\end{array}$ & $\begin{array}{l}1.414 E+04 \\
5.195 E+04\end{array}$ & $\begin{array}{l}4.712 E+04 \\
1.580 E+05\end{array}$ & $\begin{array}{l}4.455 E+04 \\
1.636 E+05\end{array}$ & $\begin{array}{l}9.167 E+04 \\
3.216 E+05\end{array}$ \\
\hline $\begin{array}{l}\text { Temperature (C) } \\
\text { Pressure (In. H2O) } \\
\text { Volume Flow (/hr) } \\
\text { Mass Flow (g/hr) } \\
\text { Solids Flow (g/hr) } \\
\text { Solids (g/) } \\
\text { Density (g/) } \\
\end{array}$ & $\begin{array}{r}20.00 \\
0.00 \\
1.185 E+05 \\
1.410 E+05\end{array}$ & $\begin{array}{r}20.02 \\
0.00 \\
1.185 \mathrm{E}+05 \\
1.410 \mathrm{E}+05 \\
\\
1.190 \mathrm{E}+00 \\
\end{array}$ & $\begin{array}{r}20.00 \\
0.00 \\
5.512 E+04 \\
6.609 E+04\end{array}$ & $\begin{array}{r}20.01 \\
0.00 \\
1.738 \mathrm{E}+05 \\
2.072 \mathrm{E}+05\end{array}$ & $\begin{array}{r}20.00 \\
0.00 \\
1.736 \mathrm{E}+05 \\
2.082 \mathrm{E}+05 \\
\\
1.199 \mathrm{E}+00 \\
\end{array}$ & $\begin{array}{r}20.01 \\
0.00 \\
3.480 E+05 \\
4.155 E+05\end{array}$ \\
\hline Phase & & Vapor & Vapor & Vapor & Vapor & Vapor \\
\hline $\begin{array}{l}\text { Flow (SCFM) } \\
\text { Temperature (K) } \\
\text { Molecular Weight } \\
\text { Prap (atm) } \\
\text { Psat (atm) } \\
\text { Dew Point (C) } \\
\text { Humidity (\%) } \\
\end{array}$ & $\begin{array}{l}6.500 E+01 \\
2.932 E+02 \\
2.861 E+01 \\
2.288 E-02 \\
2.288 E-02 \\
1.992 E+01 \\
1.000 E+02\end{array}$ & $\begin{array}{l}6.501 \mathrm{E}+01 \\
2.932 \mathrm{E}+02 \\
2.861 \mathrm{E}+01 \\
2.296 \mathrm{E} \cdot 02 \\
2.290 \mathrm{E}-02 \\
1.997 \mathrm{E}+01 \\
1.002 \mathrm{E}+02 \\
\end{array}$ & $\begin{array}{r}3.02 \mathrm{E} \\
2.932 \mathrm{E}+02 \\
2.884 \mathrm{E}+01 \\
1.125 \mathrm{E}-03 \\
2.288 \mathrm{E}-02 \\
-2.080 \mathrm{E}+01 \\
4.918 \mathrm{E}+00\end{array}$ & 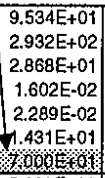 & $\begin{array}{r}9.523 E+01 \\
2.932 E+02 \\
2.884 E+01 \\
1.125 E \cdot 03 \\
2.288 E-02 \\
-2.080 E+01 \\
4.918 E+00 \\
\end{array}$ & $\begin{array}{l}1.909 \mathrm{E}+02 \\
2.932 \mathrm{E}+02 \\
2.876 \mathrm{E}+01 \\
8.574 \mathrm{E}-03 \\
2.288 \mathrm{E}-02 \\
5.042 \mathrm{E}+00 \\
3.747 \mathrm{E}+01 \\
\end{array}$ \\
\hline$\Delta \mathrm{T}(\mathrm{C})$ & & $4.265 \mathrm{E}-02$ & & $5.699 \mathrm{E}+00$ & & $1.496 \mathrm{E}+01$ \\
\hline
\end{tabular}


WSRC-TR-2000-00218

Revision 1

Page 27 of 42

Am/Cm Material Balance Spreadsheet Page 30

Version 2

\begin{tabular}{|c|c|}
\hline Melter Off-Gas & Vitrilication \\
\hline $\begin{array}{c}\text { Stream Name } \\
\text { Units } \\
\text { Stream Number }\end{array}$ & $\begin{array}{c}\text { Reaction } \\
\text { Products } \\
\text { grams } \\
76\end{array}$ \\
\hline $\begin{array}{l}\text { Gases } \\
\mathrm{H} 2 \mathrm{O} \\
\mathrm{CO} \\
\mathrm{CO} 2 \\
\mathrm{O} 2 \\
\mathrm{~N} 2 \\
\mathrm{NO}(\mathrm{NO}+\mathrm{NO} 2) / 2 \\
\mathrm{SO} 3 \\
\mathrm{POx}(\mathrm{PO} 2+\mathrm{PO} 3) / 2 \\
\mathrm{HCl} \\
\mathrm{HF}\end{array}$ & $6.329 \mathrm{E}+02$ \\
\hline
\end{tabular}

\begin{tabular}{|c|c|c|c|c|c|c|}
\hline $\begin{array}{c}\text { Stream Name } \\
\text { Units } \\
\text { Stream Number }\end{array}$ & $\begin{array}{c}\text { Sweep Air } \\
\begin{array}{c}g / h r \\
77\end{array}\end{array}$ & $\begin{array}{c}\text { Combined } \\
\text { Off-Gas } \\
g^{\prime} \mathrm{hr} \\
7 \mathrm{~g}\end{array}$ & $\begin{array}{l}\text { Dilution Air } \\
\qquad \begin{array}{l}g / h r \\
79\end{array}\end{array}$ & $\begin{array}{c}\text { Combined } \\
\text { Off-Gas } \\
9 / \mathrm{hr} \\
80\end{array}$ & $\begin{array}{c}\text { Molive Ail } \\
9 / \mathrm{hr} \\
81\end{array}$ & $\begin{array}{c}\text { Combined } \\
\text { Off-Gas } \\
g^{\prime} \mathrm{hr} \\
82\end{array}$ \\
\hline Gases & & & & & & \\
\hline $\begin{array}{l}\mathrm{H} 2 \mathrm{O} \\
\mathrm{CO} \\
\mathrm{CO} 2\end{array}$ & $2.031 E+03$ & $2.031 E+03$ & & $2.031 E+03$ & $9.947 E+01$ & $2.130 E+03$ \\
\hline 02 & $3.297 E+04$ & $3.297 E+04$ & & $3.297 E+04$ & $3.041 E+04$ & $6.338 \varepsilon+04$ \\
\hline $\begin{array}{l}\mathrm{N} 2 \\
\mathrm{NOx}(\mathrm{NO}+\mathrm{NO} 2) / 2 \\
\mathrm{SO} 3 \\
\mathrm{POx}(\mathrm{PO} 2+\mathrm{PO} 3) / 2 \\
\mathrm{HCl} \\
\mathrm{HF}\end{array}$ & $1.060 E+05$ & $1.060 E+05$ & & $1.060 E+05$ & 1.117E+05 & $2.177 E+05$ \\
\hline
\end{tabular}

\begin{tabular}{|c|c|}
\hline $\begin{array}{l}\text { Temperature (C) } \\
\text { Pressure (in. H2O) } \\
\text { Volume (l) }\end{array}$ & $\begin{array}{r}. \% 0000 \\
1.587 E+03\end{array}$ \\
\hline $\begin{array}{l}\text { Mass }(g) \\
\text { Solids }(g) \\
\text { Solids }(g /) \\
\text { Density }(g /)\end{array}$ & $6.329 \mathrm{E}+02$ \\
\hline Phase & \\
\hline
\end{tabular}

\begin{tabular}{|c|c|c|c|c|c|c|}
\hline Temperature (C) & 20.00 & 20.02 & & 26896 & 20.00 & 22.97 \\
\hline Pressure (in. $\mathrm{H} 2 \mathrm{O}$ ) & 0.0 & 0.00 & & 0.00 & 0.00 & 0.00 \\
\hline Volume Flow (/hr) & 1.185E+0: & $1.185 E+05$ & & $1.209 E+0.5$ & 1.185E+05 & $2.398 E+05$ \\
\hline Mass Flow (g/hr) & $1.410 E+05$ & 1. $410 E+05$ & & $1.410 E+05$ & $1.421 E+05$ & $2.832 E+05$ \\
\hline Solids Flow ( $g \mathrm{hr}$ ) & & & & & & \\
\hline Solids (gl) & & & & & & \\
\hline Density $(g /)$ & $1.190 E+0 C$ & $1.190 \mathrm{E}+00$ & & $1.166 \mathrm{E}+00$ & $1.199 E+00$ & $1.181 E+00$ \\
\hline Phase & Vapor & Vapor & Vapor & Vapor & Vapor & Vapor \\
\hline Flow (SCFM) & $6.500 E+01$ & $6.501 E+01$ & & $6.501 \mathrm{E}+01$ & $6.500 \mathrm{E}+01$ & $1.302 \mathrm{~F}+02$ \\
\hline Temperature $(K)$ & $2.932 \mathrm{E}+02$ & $2.932 E+02$ & & $2.991 E+02$ & $2.932 \mathrm{E}+02$ & $2.961 E+02$ \\
\hline Molecular Weight & $2.861 E+01$ & $2.861 \mathrm{E}+01$ & & $2.861 \mathrm{E}+01$ & $2.884 E+01$ & $2,873 E+01$ \\
\hline Pvap (atm) & $2.28 B E-02$ & $2.296 \mathrm{E}-02$ & & $2.296 \mathrm{E}-02$ & $1.125 \mathrm{E}-03$ & $1.204 \mathrm{E}-02$ \\
\hline Psat (atm) & $2.288 E-02$ & $2.290 \mathrm{E}-02$ & & 3.279E-02 & $2.288 \mathrm{E}-02$ & $2.742 \mathrm{E}-02$ \\
\hline Dew Point (C) & $1.992 E+01$ & $1.997 \mathrm{E}+01$ & & $1.997 \mathrm{E}+01$ & $.2 .080 E+01$ & $9.985 E+00$ \\
\hline Humidity $(\%)$ & $1.000 \mathrm{E}+02$ & $1.002 E+02$ & & $\% 000 \mathrm{~b} \times 1$ & $4.918 E+00$ & $4.390 \mathrm{E}+01$ \\
\hline$\Delta T(C)$ & & $4.265 \mathrm{E}-02$ & & $5.985 \mathrm{E}+00$ & & $1.298 \mathrm{E}+01$ \\
\hline
\end{tabular}


WSRC-TR-2000-00218, Revision 1

Page 28 of 42

\section{APPENDIX B}

Am/Cm Material Balance for 6.91 liter Batch Melter Feed Preparation Steps and Melter Operations 
WSRC-TR-2000-00218

Revision 1

Page 29 of 42

Am/Cm Material Balance Spreadsheet Page 17

Version 2

\begin{tabular}{|c|c|c|c|c|c|c|c|c|c|}
\hline \multirow[b]{2}{*}{\begin{tabular}{|c} 
Batch \\
Stream Name \\
Units \\
Stream Number
\end{tabular}} & \multicolumn{9}{|l|}{ Precipitation } \\
\hline & $\begin{array}{c}\text { Batch } \\
\text { Tank } \\
\text { grams } \\
43\end{array}$ & $\begin{array}{l}\text { MPPF } \\
\text { Feed } \\
g / 1 \\
43\end{array}$ & $\begin{array}{c}\text { Oxalic Acld } \\
8 w t \% \\
\text { grams } \\
44\end{array}$ & $\begin{array}{c}\text { Converted } \\
\text { Composition } \\
\text { grams } \\
45\end{array}$ & $\begin{array}{l}\text { Precipitate } \\
\text { grams } \\
46\end{array}$ & $\begin{array}{l}\text { Supernate } \\
\text { in Tank } \\
\text { grams } \\
47\end{array}$ & $\begin{array}{c}\text { Decanted } \\
\text { Slurry } \\
\text { grams } \\
48\end{array}$ & $\begin{array}{c}\text { Decant } \\
\text { Collection } \\
\text { grams } \\
49\end{array}$ & $\begin{array}{l}\text { Percent } \\
\text { Recovery }\end{array}$ \\
\hline Lan salts & & & & & & & & & \\
\hline $\mathrm{La}(\mathrm{NO}) 3$ & $1.98 \mathrm{tE}+02$ & $2.866 \mathrm{E}+01$ & ZERO & ZERO & ZERO & ZERO & ZERO & ZERO & \\
\hline $\mathrm{Co}(\mathrm{NO}) 3$ & $1.552 E+02$ & $2.247 E+01$ & ZERO & ZERO & ZERO & ZERO & ZERO & ZERO & \\
\hline $\operatorname{Pr}(\mathrm{NO} 3) 3$ & $1.326 \mathrm{E}+02$ & $1.919 E+01$ & ZERO & ZERO & ZERO & ZERO & ZERO & ZERO & \\
\hline $\mathrm{Nd}(\mathrm{NO} 3)_{3} 3$ & $3.762 \mathrm{E}+02$ & $5.444 E+01$ & ZERO & ZERO & ZERO & ZERO & ZERO & ZERO & \\
\hline $\mathrm{Sm}(\mathrm{NO})_{3} 3$ & $8.689 \mathrm{E}+01$ & $1.257 \mathrm{E}+01$ & ZERO & ZERO & ZERO & ZERO & ZERO & ZERO & \\
\hline Eu(NO3)3 & $1.758 E+01$ & $2.545 E+00$ & ZERO & ZERO & ZERO & ZERO & ZERO & ZERO & \\
\hline $\mathrm{Qd}(\mathrm{NO}) 3$ & $4.385 E+01$ & $6.345 \mathrm{E}+00$ & ZERO & ZERO & ZERO & ZERO & ZERO & ZERO & \\
\hline $\mathrm{Tb}(\mathrm{NO}) 3$ & $5.516 \mathrm{E}+00$ & $7.982 \mathrm{E}-01$ & ZERO & ZERO & ZERO & ZERO & ZERO & ZERO & \\
\hline$D y(N O 3) 3$ & $5.449 \mathrm{E}+\infty$ & $7.885 \mathrm{E}-01$ & ZERO & ZERO & ZERO & ZERO & ZERO & ZERO & \\
\hline $\mathrm{Ho}(\mathrm{NO} 3)_{3} 3$ & $5.405 \mathrm{E}+00$ & $7.822 \mathrm{E}-01$ & ZERO & ZERO & ZERO & ZERO & ZERO & ZERO & \\
\hline $\mathrm{Er}(\mathrm{NOS}) 3$ & $5.364 E+00$ & $7.762 \mathrm{E}-01$ & ZERO & ZERO & ZERO & ZERO & ZERO & ZERO & \\
\hline $\operatorname{Tm}(\mathrm{NO}) 3$ & $7.179 E+00$ & $1.039 \mathrm{E}+00$ & ZERO & ZERO & ZERO & ZERO & ZERO & ZERO & \\
\hline $\mathrm{Yb}(\mathrm{NO}) 3$ & $5.267 \mathrm{E}+00$ & $7.622 E-01$ & ZERO & ZERO & ZERO & ZERO & ZERO & ZERO & \\
\hline LU(NO3)3 & $5.236 \mathrm{E}+00$ & $7.577 \mathrm{E}-01$ & ZERO & ZERO & ZERO & ZERO & ZERO & ZERO & \\
\hline $\begin{array}{l}\text { Lan oxalates } \\
\mathrm{Le}(\mathrm{C} 2 \mathrm{O} 4) 3\end{array}$ & & & & & & & & & \\
\hline Le2(C2O4)3 & ZERO & ZERO & ZERO & $1.651 E+02$ & $1.434 E+02$ & $2.180 \varepsilon+01$ & $1.452 E+02$ & $1.998 \mathrm{E}+01$ & 87.90 \\
\hline $\mathrm{Ce} 2(\mathrm{C} 2 \mathrm{O} 4) 3$ & ZERO & ZERO & ZERO & 1.296E+02 & $1.238 \mathrm{E}+02$ & $5.736 \mathrm{E}+00$ & $1.243 E+02$ & $5.259 \mathrm{E}+00$ & 95.94 \\
\hline $\begin{array}{l}\mathrm{Pr}(\mathrm{C} 2 \mathrm{O} 4) 3 \\
\mathrm{Nd} 2(\mathrm{C} 2 \mathrm{O} 4) 3\end{array}$ & $\begin{array}{l}\text { ZERO } \\
\text { ZERO }\end{array}$ & $\begin{array}{l}\text { ZERO } \\
\text { ZERO }\end{array}$ & $\begin{array}{l}\text { ZERO } \\
\text { ZERO }\end{array}$ & $\begin{array}{l}1.107 \mathrm{E}+02 \\
3.147 \mathrm{E}+02\end{array}$ & $\begin{array}{l}1.076 \mathrm{E}+02 \\
3.116 \mathrm{E}+02\end{array}$ & $\begin{array}{l}3.058 \mathrm{E}+\infty 0 \\
3.071 \mathrm{E}+\infty\end{array}$ & $\begin{array}{l}1.079 E+02 \\
3.119 E+02\end{array}$ & $\begin{array}{l}2.804 E+00 \\
2.816 E+00\end{array}$ & $\begin{array}{l}97.47 \\
99.11\end{array}$ \\
\hline $\mathrm{Sm} 2(\mathrm{C} 2 \mathrm{O} 4)^{3}$ & ZERO & ZERO & ZERO & $7.295 E+01$ & $7.004 E+01$ & $2.905 E+00$ & $7.028 E+01$ & $2.664 E+00$ & 96.35 \\
\hline $\mathrm{Eu} 2(\mathrm{C2O} 4) 3$ & ZERO & ZERO & ZERO & $1.478 \mathrm{E}+01$ & $1.453 E+01$ & $2.412 E-01$ & 1.455E+01 & 2.212E-01 & 98.50 \\
\hline $\mathrm{Gd} 2(\mathrm{C} 2 \mathrm{O} 4) 3$ & ZERO & ZERO & ZERO & $3.695 \mathrm{E}+01$ & $3.679 E+01$ & $1.600 E-01$ & $3.680 E+01$ & $1.467 \mathrm{E}-01$ & 99.60 \\
\hline $\mathrm{Tb} 2(\mathrm{C} 2 \mathrm{O} 4) 3$ & ZERO & ZERO & ZERO & $4.653 E+00$ & $3.205 \mathrm{E}+00$ & $1.448 \mathrm{E}+00$ & $3.325 \mathrm{E}+00$ & $1.327 \mathrm{E}+00$ & 71.47 \\
\hline Dy2 $\left(\mathrm{C}^{2} \mathrm{O} 4\right) 3$ & ZERO & ZERO & ZERO & $4.605 E+00$ & $3.157 \mathrm{E}+00$ & $1.448 E+00$ & $3.277 \mathrm{E}+00$ & $1.327 E+00$ & 71.18 \\
\hline $\mathrm{Ho} 2(\mathrm{C} \mathrm{O} 4)^{3}$ & ZERO & ZERO & ZERO & $4.573 E+00$ & $3.126 E+00$ & $1.448 E+00$ & $3.246 E+00$ & $1.327 E+00$ & 70.98 \\
\hline $\mathrm{Er} 2(\mathrm{C} 2 \mathrm{O} 4) 3$ & ZERO & ZERO & ZERO & $4.544 E+00$ & $3.097 E+00$ & $1.448 E+00$ & $3.217 \mathrm{E}+00$ & $1.327 \mathrm{E}+00$ & 70.79 \\
\hline $\operatorname{Tm} 2(\mathrm{C} 2 \mathrm{O} 4)^{3}$ & ZERO & ZERO & ZERO & $6.087 E+00$ & $4.639 E+00$ & $1.448 E+00$ & $4.760 \mathrm{E}+00$ & $1.327 E+00$ & 78.20 \\
\hline $\mathrm{Yb} 2(\mathrm{C} 2 \mathrm{O} 4) 3$ & ZERO & ZERO & ZERO & $4.475 E+\infty$ & $3.027 \mathrm{E}+00$ & $1.448 \mathrm{E}+00$ & $3.148 E+\infty 0$ & $1.327 \mathrm{E}+00$ & 70.34 \\
\hline $\begin{array}{l}\text { Lu2(C2O4)3 } \\
\text { Act salts }\end{array}$ & ZERO & ZERO & ZERO & $4.453 E+00$ & $3.005 E+00$ & $1.448 \mathrm{E}+00$ & $3.126 \mathrm{E}+\infty 0$ & $1.327 \mathrm{E}+\infty$ & 70.19 \\
\hline Am(NO3)3 & $9.041 E+01$ & $1.308 \mathrm{E}+01$ & ZERO & ZERO & ZERO & ZERO & ZERO & ZERO & \\
\hline $\mathrm{Cm}(\mathrm{NO} 3)_{3}$ & $2.218 \mathrm{E}+01$ & $3.210 E+00$ & ZERO & ZERO & ZERO & ZERO & ZERO & ZERO & \\
\hline $\mathrm{NpO} 2$ (NO3) & $3.630 \mathrm{E}-03$ & $5.253 \mathrm{E}-04$ & ZERO & ZERO & ZERO & ZERO & ZERO & ZERO & \\
\hline $\mathrm{Pu}(\mathrm{NO} 3)_{4}$ & $2.540 E+01$ & $3.676 \mathrm{E}+00$ & ZERO & ZERO & ZERO & ZERO & ZERO & ZERO & \\
\hline บO2(NO3)2 & $1.521 E+00$ & $2.201 \mathrm{E}-01$ & ZERO & ZERO & ZERO & ZERO & ZERO & ZERO & \\
\hline $\begin{array}{l}\text { CsNO3 } \\
\text { Act oxalates }\end{array}$ & $7.849 \mathrm{E}-03$ & $1.136 \mathrm{E}-03$ & ZERO & ZERO & ZERO & ZERO & ZERO & ZERO & \\
\hline $\operatorname{Am} 2\left(C_{2} O 4\right) 3$ & ZERO & ZERO & ZERO & $7.903 \mathrm{E}+01$ & $7.889 \mathrm{E}+01$ & $1.470 E-01$ & $7.890 E+01$ & $1.348 \mathrm{E}-01$ & 99.83 \\
\hline $\mathrm{Cm} 2(\mathrm{C} 2 \mathrm{O} 4) 3$ & ZERO & ZERO & ZERO & $1.940 E+01$ & $1.931 E+01$ & $8.870 \mathrm{E}-02$ & $1.932 \mathrm{E}+01$ & $8.133 \mathrm{E}-02$ & 99.58 \\
\hline $\mathrm{Np}_{2} \mathrm{O}_{4}\left(\mathrm{C} 2 \mathrm{O}_{4}\right)$ & ZERO & ZERO & ZERO & 3.433E-03 & $0.000 \mathrm{E}+00$ & $3.433 \mathrm{E}-03$ & 2.854E-04 & $3.147 \mathrm{E} \cdot 03$ & 8.31 \\
\hline $\mathrm{Pu}(\mathrm{C} 2 \mathrm{O} 4) 2$ & ZERO & ZERO & ZERO & $2.166 \mathrm{E}+01$ & $2.155 E+01$ & $1.038 \mathrm{E} \cdot 01$ & $2.156 E+01$ & $9.516 \mathrm{E}-02$ & 99.56 \\
\hline $\mathrm{UO}_{2}\left(\mathrm{C}_{2} \mathrm{O} 4\right)$ & ZERO & ZERO & ZERO & $1.382 E+\infty 0$ & $0.000 \mathrm{E}+00$ & $.1 .382 E+00$ & $1.149 \mathrm{E}-01$ & $1.267 \mathrm{E}+00$ & 8.31 \\
\hline $\mathrm{Cs} 2 \mathrm{C} 2 \mathrm{O} 4$ & ZERO & ZERO & ZERO & $7.125 \mathrm{E}-03$ & $0.000 E+00$ & $7.125 \mathrm{E}-03$ & $5.922 \mathrm{E}-04$ & $6.532 \mathrm{E} \cdot 03$ & 8.31 \\
\hline
\end{tabular}


WSRC-TR-2000-00218

Revision 1

Page 30 of 42

Am/Cm Material Balance Spreadsheet Page 18

Version 2

\begin{tabular}{|c|c|c|c|c|c|c|c|c|c|}
\hline $\begin{array}{c}\text { Stream Name } \\
\text { Units } \\
\text { Stream Number }\end{array}$ & $\begin{array}{c}\text { Batch } \\
\text { Tank } \\
\text { grams } \\
43\end{array}$ & $\begin{array}{c}\text { MPPF } \\
\text { Feed } \\
91 \\
43 \\
\end{array}$ & $\begin{array}{c}\text { Oxalic Acid } \\
\text { 8 wt } \% \\
\text { grams } \\
44\end{array}$ & $\begin{array}{c}\text { Converted } \\
\text { Composition } \\
\text { grams } \\
45 \\
\end{array}$ & $\begin{array}{l}\text { Preclpitate } \\
\text { grams } \\
46\end{array}$ & $\begin{array}{c}\text { Supernate } \\
\text { in Tank } \\
\text { grams } \\
47\end{array}$ & $\begin{array}{c}\text { Decanted } \\
\text { Slumy } \\
\text { grams } \\
48 \\
\end{array}$ & $\begin{array}{l}\text { Decant } \\
\text { Collection } \\
\text { grams } \\
49\end{array}$ & $\begin{array}{l}\text { Percent } \\
\text { Recovery }\end{array}$ \\
\hline \multicolumn{10}{|l|}{ Metallic salts } \\
\hline $\mathrm{Al} 2(\mathrm{SO} 4) 3$ & $6.266 \mathrm{E}+00$ & $9.068 \mathrm{E}-01$ & ZERO & $6.266 \mathrm{E}+00$ & ZERO & $6.266 \mathrm{E}+00$ & $5.208 \mathrm{E}-01$ & $5.745 \mathrm{E}+00$ & 8.31 \\
\hline AlF3 & $4.614 \mathrm{E}-01$ & 6.677E-02 & ZERO & $4.614 \mathrm{E}-01$ & ZERO & 4.614E-01 & $3.835 \mathrm{E}-02$ & $4.230 \mathrm{E}-01$ & 8.31 \\
\hline $\mathrm{Al}(\mathrm{NO} 3)_{3}$ & $4.031 E+00$ & 5.833E-01 & ZERO & $4.031 E+00$ & ZERO & $4.031 E+00$ & $3.350 E-01$ & $3.696 E+00$ & 8.31 \\
\hline $\mathrm{Ca}(\mathrm{NO} 3)_{2}$ & 4.105E-01 & $5.940 \mathrm{E}-02$ & ZERO & $4.105 E-01$ & ZERO & $4.105 \mathrm{E}-01$ & $3.412 E-02$ & $3.763 \mathrm{E}-01$ & 8.31 \\
\hline $\mathrm{Cr}(\mathrm{NO}) 3$ & $2.714 E+00$ & $3.928 \mathrm{E}-01$ & ZERO & $2.714 E+\infty 0$ & ZERO & $2.714 E+00$ & $2.256 \mathrm{E}-01$ & $2.489 E+\infty 0$ & 8.31 \\
\hline $\mathrm{Fe} 2(\mathrm{SO} 4) 3$ & $1.266 \mathrm{E}+01$ & $1.832 E+\infty 0$ & ZERO & $1.266 \mathrm{E}+01$ & ZERO & $1.266 \mathrm{E}+01$ & $1.052 E+00$ & $1.161 \mathrm{E}+01$ & 8.31 \\
\hline FoPO4 & $2.861 E+\infty 0$ & 4.140E-01 & ZERO & $2.861 E+\infty$ & ZERO & $2.861 E+\infty$ & $2.378 \mathrm{E}-01$ & $2.623 \mathrm{E}+\infty 0$ & 8.31 \\
\hline $\mathrm{FeCl3}$ & $2.228 E+00$ & $3.225 \mathrm{E}-01$ & ZERO & $2.228 \mathrm{E}+00$ & ZERO & $2.228 \mathrm{E}+00$ & $1.852 \mathrm{E}-01$ & $2.043 E+00$ & 8.31 \\
\hline $\mathrm{F} \theta 2(\mathrm{C} 2 \mathrm{O} 4) 3$ & $2.458 E+00$ & $3.557 \mathrm{E}-01$ & ZERO & $2.458 E+00$ & ZERO & $2.458 E+00$ & $2.043 \mathrm{E}-01$ & $2.254 E+00$ & 8.31 \\
\hline $\mathrm{Fe}(\mathrm{NO}) 3$ & $5.252 E+\infty 0$ & $7.601 E-01$ & ZERO & $5.252 E+00$ & ZERO & $5.252 E+00$ & 4.366E-01 & $4.816 E+00$ & 8.31 \\
\hline KNO3 & $8.446 \mathrm{E}-01$ & $1.222 E-01$ & ZERO & $8.446 \mathrm{E}-01$ & ZERO & $8.446 \mathrm{E}-01$ & $7.021 \mathrm{E}-02$ & 7.744E-01 & 8.31 \\
\hline $\mathrm{Mn}(\mathrm{NO}) 2$ & $1.349 \mathrm{E}+02$ & $1.952 E+01$ & ZERO & $1.349 E+02$ & ZERO & $1.349 \mathrm{E}+02$ & $1.121 E+01$ & $1.237 \mathrm{E}+02$ & 8.31 \\
\hline $\mathrm{NaNO3}$ & $9.920 \mathrm{E}-01$ & $1.436 \mathrm{E}-01$ & ZERO & $9.920 \mathrm{E}-01$ & ZERO & $9.920 \mathrm{E}-01$ & 8.246E-02 & $9.095 \mathrm{E} \cdot 01$ & 8.31 \\
\hline $\mathrm{NaNO} 2$ & $1.528 \mathrm{E}+00$ & 2.212E-01 & ZERO & $1.528 E+00$ & ZERO & $1.528 E+00$ & $1.270 \mathrm{E}-01$ & $1.401 E+00$ & 8.31 \\
\hline $\mathrm{Ne} 2 \mathrm{~B} 4 \mathrm{O} 7$ & $5.828 E-02$ & $8.434 \mathrm{E}-03$ & ZERO & $5.828 \mathrm{E}-02$ & ZERO & $5.828 \mathrm{E}-02$ & 4.844E-03 & $5.343 E-02$ & 8.31 \\
\hline $\mathrm{Na2SIO3}$ & $9.618 \mathrm{E}-02$ & $1.392 \mathrm{E}-02$ & ZERO & $9.618 \mathrm{E}-02$ & ZERO & $9.618 \mathrm{E}-02$ & 7.995E-03 & $8.818 \mathrm{E}-02$ & 8.31 \\
\hline $\mathrm{N} /(\mathrm{NO}) 2$ & $1.351 E+\infty 0$ & $1.955 \mathrm{E}-01$ & ZERO & $1.351 E+00$ & ZERO & $1.351 E+00$ & $1.123 \mathrm{E}-01$ & $1.239 E+00$ & 8.31 \\
\hline $\mathrm{Zn}(\mathrm{NO} 3) 2$ & $9.245 \mathrm{E}-02$ & $1.338 \mathrm{E}-02$ & ZERO & $9.245 \mathrm{E}-02$ & ZERO & $9.245 \mathrm{E}-02$ & 7.685E-03 & 8.477E-02 & 8.31 \\
\hline $\mathrm{Zr}(\mathrm{NO3}) 4$ & 2.277E-02 & $3.295 \mathrm{E}-03$ & ZERO & $2.277 \mathrm{E}-02$ & ZERO & $2.277 \mathrm{E}-02$ & 1.892E-03 & $2.087 \mathrm{E}-02$ & 8.31 \\
\hline \multicolumn{10}{|l|}{ Miscollanøous } \\
\hline insoluble sollds & ZERO & ZERO & ZERO & ZERO & ZERO & ZERO & ZERO & ZERO & \\
\hline HNO3 & $4.354 E+02$ & $6.301 E+01$ & ZERO & $1.100 E+03$ & ZERO & $1.100 E+03$ & $9.142 E+01$ & $1.008 E+03$ & \\
\hline $\mathrm{HCOOH}$ & ZERO & ZERO & ZERO & ZERO & ZERO & ZERO & ZERO & ZERO & \\
\hline $\mathrm{H} 2 \mathrm{C} 2 \mathrm{O} 4$ & ZERO & ZERO & $9.978 E+02$ & $5.232 E+02$ & ZERO & $5.232 E+02$ & $4.349 E+01$ & $4.797 E+02$ & \\
\hline $\mathrm{CO}^{\mathrm{H}}$ & ZERO & ZERO & ZERO & ZERO & ZERO & ZERO & ZERO & ZERO & \\
\hline $\mathrm{O} 2$ & ZERO & ZERO & ZERO & ZERO & ZERO & ZERO & ZERO & ZERO & \\
\hline N2 & ZERO & ZERO & ZERO & ZERO & ZERO & ZERO & ZERO & ZERO & \\
\hline NOX & ZERO & ZERO & ZERO & ZERO & ZERO & ZERO & ZERO & ZERO & \\
\hline $\mathrm{H} 2 \mathrm{O}$ & $6.714 E+03$ & $9.717 \mathrm{E}+02$ & $1.148 E+04$ & $1.819 E+04$ & ZERO & $1.819 E+04$ & $1.512 E+03$ & $1.668 E+04$ & \\
\hline Nitrle Acld $(M)$ & $1.000 E+00$ & & ZERO & $9.218 E-01$ & ZERO & $9.672 \mathrm{E}-01$ & $6.074 \mathrm{E}-01$ & $9.672 \mathrm{E} \cdot 01$ & \\
\hline Oxallic Acld (M) & ZERO & & $9.218 \mathrm{E}-01$ & $3.069 \mathrm{E}-01$ & ZERO & $3.220 \mathrm{E}-01$ & $2.022 \mathrm{E}-01$ & $3.220 \mathrm{E}-01$ & \\
\hline \multicolumn{10}{|l|}{$\mathrm{Mn}+2$ (M) } \\
\hline Heat $(W)$ & $3.527 E+01$ & & & $3.527 \mathrm{E}+01$ & & $3.527 E+01$ & $3.512 \mathrm{E}+01$ & $1.466 \mathrm{E}-01$ & \\
\hline$G(\mathrm{H} 2)$ & $6.575 E-01$ & & & $6.820 \mathrm{E}-01$ & & $6.675 \mathrm{E}-01$ & $8.023 E-01$ & $6.675 \mathrm{E}-01$ & \\
\hline $\mathrm{H} 2(\mathrm{~g}-\mathrm{mole} / \mathrm{hr})$ & $8.652 E-03$ & & & $8.974 \mathrm{E}-03$ & & $8.784 E-03$ & $1.051 \mathrm{E}-02$ & $3.652 E-05$ & \\
\hline Alr Purge (SCFM) & $1.102 E-02$ & & & $1.143 \mathrm{E}-02$ & & $1.119 \mathrm{E}-02$ & $1,339 \mathrm{E}-02$ & 4.652E-05 & \\
\hline Volume (l) & $6.910 \mathrm{E}+00$ & & $1.202 E+01$ & $1.893 \mathrm{E}+01$ & $8.886 \mathrm{E}-01$ & $1.804 E+01$ & $2.389 E+00$ & $1.654 \mathrm{E}+01$ & \\
\hline Mass (kg) & $8.518 E+00$ & & $1.247 \mathrm{E}+01$ & $2.099 E+01$ & $9.508 \mathrm{E}-01$ & $2.004 E+01$ & $2.617 E+\infty 0$ & $1.837 E+01$ & \\
\hline Solids $(g)$ & $1.369 \varepsilon+03$ & & ZERO & $1.179 E+03$ & $9.508 E+02$ & $2.280 E+02$ & $9.698 \mathrm{E}+02$ & $2.001 E+02$ & \\
\hline Solids $(g \mid)$ & $1.981 E+02$ & & ZERO & $6.226 E+01$ & $1.070 E+03$ & $1.264 E+01$ & $4.060 \mathrm{E}+02$ & 1. $264 \mathrm{E}+01$ & \\
\hline Density $(\mathrm{g} / \mathrm{ml})$ & $1.233 E+00$ & & $1.037 E+00$ & $1.109 E+00$ & $1.070 E+00$ & $1.111 \mathrm{E}+00$ & $1.096 \mathrm{E}+00$ & $1.111 E+\infty$ & \\
\hline Phase & Liquid & Liquid & Liquidd & Liquild & Solld & Liquid & Liquid & Llquld & \\
\hline & & & $\begin{array}{c}\text { Volume Ratio } \\
1.740\end{array}$ & $\begin{array}{l}\text { Moles Precip } \\
1.777 \mathrm{E}+00\end{array}$ & & & $\begin{array}{l}\text { lume Ratio } \\
8.313 \mathrm{E}-02\end{array}$ & & \\
\hline
\end{tabular}


WSRC-TR-2000-00218

Revision 1

Page 31 of 42

Am/Cm Material Balance Spreadsheet Page 19

\begin{tabular}{|c|c|c|c|c|c|c|c|}
\hline $\begin{array}{c}\text { Stream Name } \\
\text { Units } \\
\text { Stream Number }\end{array}$ & $\begin{array}{l}\text { Oxalic Acid } \\
0.1 \mathrm{M} \\
\text { grams } \\
50\end{array}$ & $\begin{array}{l}\text { Slurry } \\
\text { and Wash } \\
\text { grams } \\
51\end{array}$ & $\begin{array}{l}\text { Precipitate } \\
\text { grams } \\
52\end{array}$ & $\begin{array}{l}\text { Supemate } \\
\text { In Tank } \\
\text { grams } \\
53\end{array}$ & $\begin{array}{c}\text { Decanted } \\
\text { Slury } \\
\text { grams } \\
54\end{array}$ & $\begin{array}{l}\text { Decant } \\
\text { Collection } \\
\text { grams } \\
55\end{array}$ & $\begin{array}{c}\text { Percent } \\
\text { Recovery }\end{array}$ \\
\hline Lan salts & & & & ZERO & ZERO & ZERO & \\
\hline $\begin{array}{l}\mathrm{La}(\mathrm{NO}) 3 \\
\mathrm{Co}(\mathrm{NO}) 3\end{array}$ & $\begin{array}{l}\text { ZERO } \\
\text { ZERO }\end{array}$ & $\begin{array}{l}\text { ZERO } \\
\text { ZERO }\end{array}$ & $\begin{array}{l}\text { ZERO } \\
\text { ZERO }\end{array}$ & $\begin{array}{l}\text { ZERO } \\
\text { ZERO }\end{array}$ & ZERO & ZERO & \\
\hline $\operatorname{Pr}(\mathrm{NO} 3) 3$ & ZERO & ZERO & ZERO & ZERO & ZERO & ZERO & \\
\hline $\mathrm{Nd}(\mathrm{NO})_{3} 3$ & ZERO & ZERO & ZERO & ZERO & ZERO & ZERO & \\
\hline $\mathrm{Sm}(\mathrm{NO}) 3$ & ZERO & ZERO & ZERO & ZERO & ZERO & ZERO & \\
\hline Eu(NO3)3 & ZERO & ZERO & ZERO & ZERO & ZERO & ZERO & \\
\hline Gd(NO3)3 & ZERO & ZERO & ZERO & ZERO & ZERO & ZERO & \\
\hline $\mathrm{Tb}(\mathrm{NO}) 3$ & ZERO & ZERO & ZERO & ZERO & ZERO & ZERO & \\
\hline Dy $(\mathrm{NO} 3)^{3}$ & ZERO & ZERO & ZERO & ZERO & ZERO & ZERO & \\
\hline $\mathrm{Ho}(\mathrm{NO}) 3$ & ZERO & ZERO & ZERO & ZERO & ZERO & ZERO & \\
\hline $\mathrm{Er}(\mathrm{NO}) 3$ & ZERO & ZERO & ZERO & ZERO & ZERO & ZERO & \\
\hline $\operatorname{Tm}(\mathrm{NO} 3)_{3}$ & ZERO & ZERO & ZERO & ZERO & ZERO & ZERO & \\
\hline Yb(NO3)3 & ZERO & ZERO & ZERO & ZERO & ZERO & ZERO & \\
\hline Lu(NO3) 3 & ZERO & ZERO & ZERO & ZERO & ZERO & ZERO & \\
\hline Lan oxalates & & & & & & & \\
\hline $\mathrm{La2}(\mathrm{C} 2 \mathrm{O} 4) 3$ & ZERO & $1.452 E+02$ & $1.452 E+02$ & $7.347 \mathrm{E}-03$ & $1.452 E+02$ & $6.007 \mathrm{E}-03$ & 87.90 \\
\hline $\mathrm{Ce} 2(\mathrm{C} 2 \mathrm{O} 4) 3$ & ZERO & $1.243 E+02$ & $1.243 E+02$ & $4.685 \mathrm{E}-03$ & $1.243 E+02$ & $3.831 \mathrm{E}-03$ & 95.94 \\
\hline $\mathrm{Pr} 2\left(\mathrm{C}_{2} \mathrm{O} 4\right) 3$ & ZERO & $1.079 E+02$ & $1.079 E+02$ & $1.390 \mathrm{E}-03$ & $1.079 E+02$ & $1.136 \mathrm{E}-03$ & 97.47 \\
\hline $\mathrm{Nd} 2(\mathrm{C} 2 \mathrm{O} 4) 3$ & ZERO & $3.119 E+02$ & $3.119 \varepsilon+02$ & 2.127E-03 & 3.t19E+02 & $1.739 \mathrm{E}-03$ & 99.10 \\
\hline $\mathrm{Sm} 2(\mathrm{c} 2 \mathrm{O} 4) 3$ & ZERO & $7.028 E+01$ & $7.028 E+01$ & 2.122E-03 & $7.028 \mathrm{E}+01$ & $1.735 \mathrm{E}-03$ & 96.35 \\
\hline Eu2(C2O4)3 & ZERO & $1.455 E+01$ & $1.455 E+01$ & $2.200 \mathrm{E}-04$ & $1.455 E+01$ & $1.799 \mathrm{E}-04$ & 98.50 \\
\hline $\mathrm{Gd} 2(\mathrm{C} 2 \mathrm{O} 4) 3$ & ZERO & $3.680 \mathrm{E}+01$ & $3.680 E+01$ & $2.776 \mathrm{E}-04$ & $3.680 E+01$ & $2.270 \mathrm{E}-04$ & 99.60 \\
\hline $\mathrm{To} 2(\mathrm{C2O} 4) 3$ & ZERO & $3.325 \mathrm{E}+00$ & $3.324 E+00$ & $1.182 E-03$ & $3.324 E+00$ & $9.669 \mathrm{E}-04$ & 71.45 \\
\hline $\mathrm{Dy} 2(\mathrm{C} 2 \mathrm{O} 4) 3$ & ZERO & $3.277 E+00$ & $3.276 E+00$ & $1.182 E-03$ & $3.277 E+00$ & $9.669 \mathrm{E}-04$ & 71.16 \\
\hline $\mathrm{Ho} 2\left(\mathrm{C}_{2} \mathrm{O} 4\right) 3$ & ZERO & $3.246 \mathrm{E}+00$ & $3.245 E+00$ & $1.182 E-03$ & $3.245 E+00$ & $9.669 \mathrm{E}-04$ & 70.96 \\
\hline $\mathrm{Er} 2(\mathrm{C} 2 \mathrm{O} 4) 3$ & ZERO & $3.217 \mathrm{E}+00$ & $3.216 E+00$ & 1. $182 E-03$ & $3.216 E+00$ & $9.669 \mathrm{E}-04$ & 70.77 \\
\hline $\operatorname{Tm} 2\left(\mathrm{C}_{2} \mathrm{O}\right) 3$ & ZERO & $4.760 E+00$ & $4.758 E+00$ & $1.182 \mathrm{E}-03$ & $4.759 E+00$ & $9.669 \mathrm{E}-04$ & 78.18 \\
\hline $\mathrm{Yb} 2(\mathrm{C2O} 4) 3$ & ZERO & $3.148 E+00$ & $3.147 E+\infty 0$ & $1.102 \mathrm{E}-03$ & $3.147 E+\infty$ & $9.669 \mathrm{E}-04$ & 70.32 \\
\hline $\begin{array}{l}\text { Lu2(C2O4)3 } \\
\text { Act salts }\end{array}$ & ZERO & $3.126 \mathrm{E}+00$ & $3.125 E+00$ & $1.182 E-03$ & $3.125 E+00$ & $9.669 \mathrm{E}-04$ & 70.17 \\
\hline $\mathrm{Am}(\mathrm{NO}) 3$ & ZERO & ZERO & ZERO & ZERO & ZERO & ZERO & \\
\hline $\mathrm{Cm}(\mathrm{NO} 3)_{3} 3$ & ZERO & ZERO & ZERO & ZERO & ZERO & ZERO & \\
\hline $\mathrm{NpO} 2(\mathrm{NO} 3)$ & ZERO & ZEAO & ZERO & ZERO & ZERO & ZERO & \\
\hline $\mathrm{Pu}(\mathrm{NO3}) 4$ & ZERO & ZERO & ZERO & ZERO & ZERO & ZERO & \\
\hline VO2(NO3)2 & ZERO & ZERO & ZERO & ZERO & ZERO & ZERO & \\
\hline $\begin{array}{l}\text { CsNO3 } \\
\text { Act oxalates }\end{array}$ & ZERO & ZERO & ZERO & ZERO & ZERO & ZERO & \\
\hline $\operatorname{Am} 2(\mathrm{C} 2 \mathrm{O} 4) 3$ & ZERO & $7.890 \mathrm{E}+01$ & $7.889 \mathrm{E}+01$ & $1.212 \mathrm{E}-02$ & $7.889 E+01$ & $9.913 \mathrm{E}-03$ & 99.82 \\
\hline $\mathrm{Cm} 2\left(\mathrm{C}^{2} \mathrm{O} 4\right) 3$ & ZERO & $1.932 \mathrm{E}+01$ & $1.931 E+01$ & $6.088 \mathrm{E}-03$ & $1.931 E+01$ & $4.962 \mathrm{E}-03$ & 99.56 \\
\hline $\mathrm{Np} 2 \mathrm{O} 4(\mathrm{C} 2 \mathrm{O} 4)$ & ZERO & 2.854E-04 & $0.000 \mathrm{E}+00$ & $2.854 \mathrm{E}-04$ & $5.203 E-05$ & $2.333 \mathrm{E}-04$ & 1.52 \\
\hline $\mathrm{Pu}(\mathrm{C} 2 \mathrm{O} 4)_{2}$ & ZERO & $2.156 \mathrm{E}+01$ & $2.149 \mathrm{E}+01$ & $7.287 \mathrm{E}-02$ & $2.150 \mathrm{E}+01$ & $5.959 \mathrm{E}-02$ & 99.29 \\
\hline $\mathrm{UO} 2\left(\mathrm{C}_{2} \mathrm{O}_{4}\right)$ & ZERO & $1.149 \mathrm{E}-01$ & $0.000 E+\infty 0$ & $1.149 \mathrm{E}-01$ & $2.095 \mathrm{E}-02$ & 9.393E-02 & 1.52 \\
\hline $\mathrm{Cs} 2 \mathrm{C} 2 \mathrm{O} 4$ & ZERO & $5.922 \mathrm{E}-04$ & $0.000 \mathrm{E}+00$ & $5.922 \mathrm{E}-04$ & $1.080 \mathrm{E}-04$ & $4.843 \mathrm{E}-04$ & 1.52 \\
\hline
\end{tabular}


WSRC-TR-2000-00218

Revision 1

Version 2

Am/Cm Material Balance Spreadsheet Page 20

\begin{tabular}{|c|c|c|c|c|c|c|c|}
\hline $\begin{array}{c}\text { Stream Name } \\
\text { Unlts } \\
\text { Stream Number }\end{array}$ & $\begin{array}{l}\text { Oxalic Acld } \\
0.1 \mathrm{M} \\
\text { grams } \\
50\end{array}$ & $\begin{array}{c}\text { Slurry } \\
\text { and Wash } \\
\text { grams } \\
51\end{array}$ & $\begin{array}{l}\text { Precipitate } \\
\text { grams } \\
52\end{array}$ & $\begin{array}{l}\text { Supemate } \\
\text { in Tank } \\
\text { grams } \\
53\end{array}$ & $\begin{array}{l}\text { Decanted } \\
\text { Slurry } \\
\text { grams } \\
54\end{array}$ & $\begin{array}{l}\text { Decant } \\
\text { Collectlon } \\
\text { grams } \\
55\end{array}$ & $\begin{array}{l}\text { Percent } \\
\text { Recovery }\end{array}$ \\
\hline \multicolumn{8}{|l|}{ Metallic salts } \\
\hline $\mathrm{Al} 2(\mathrm{SO} 4) 3$ & ZERO & $5.208 \mathrm{E}-01$ & ZERO & 5.208E-01 & $9.497 \mathrm{E}-02$ & 4.259E-01 & 1.52 \\
\hline AlF3 & ZERO & 3.835E-02 & ZERO & $3.835 \mathrm{E}-02$ & $6.993 \mathrm{E}-03$ & $3.136 \mathrm{E}-02$ & 1.52 \\
\hline $\mathrm{Al}(\mathrm{NO}$ 3)3 & ZERO & $3.350 E-01$ & ZERO & $3.350 \mathrm{E}-01$ & $6.109 \mathrm{E}-02$ & $2.740 \mathrm{E}-01$ & 1.52 \\
\hline $\mathrm{Ca}(\mathrm{NO} 3) 2$ & ZERO & 3.412E-02 & ZERO & 3.412E-02 & $6.221 \mathrm{E}-03$ & $2.790 \mathrm{E}-02$ & 1.52 \\
\hline $\mathrm{Cr}(\mathrm{NO}) 3$ & ZERO & $2.256 \mathrm{E}-01$ & ZERO & $2.256 \mathrm{E}-01$ & 4.114E-02 & $1.845 \mathrm{E}-01$ & 1.52 \\
\hline $\mathrm{Fe} 2(\mathrm{SO} 4) 3$ & ZERO & $1.052 E+00$ & ZERO & $1.052 E+\infty 0$ & $1.919 E-01$ & $8.605 \mathrm{E}-01$ & 1.52 \\
\hline FePO4 & ZERO & 2.378E-01 & ZERO & 2.378E-01 & $4.336 \mathrm{E}-02$ & $1.945 \mathrm{E}-01$ & 1.52 \\
\hline $\mathrm{FeCl} 3$ & ZERO & $1.852 \mathrm{E}-01$ & ZERO & $1.852 \mathrm{E}-01$ & 3.377E-02 & $1.514 \mathrm{E}-01$ & 1.52 \\
\hline $\mathrm{Fe} 2(\mathrm{C} 2 \mathrm{O} 4) 3$ & ZERO & $2.043 E-01$ & ZERO & 2.043E-01 & $3.725 \mathrm{E}-02$ & 1.671E-01 & 1.52 \\
\hline $\mathrm{Fa}(\mathrm{NO}) 3$ & ZERO & 4.366E-01 & ZERO & 4.366E-01 & $7.961 \mathrm{E}-02$ & $3.570 \mathrm{E}-01$ & 1.52 \\
\hline KNO3 & ZERO & $7.021 \varepsilon-02$ & ZERO . & $7.021 \mathrm{E}-02$ & 1.280E-02 & $5.741 \mathrm{E}-02$ & 1.52 \\
\hline $\mathrm{Mn}(\mathrm{NO}) 2$ & ZERO & $1.121 E+01$ & ZERO & 1.121E+01 & $2.044 E+\infty 0$ & $9.168 \mathrm{E}+\infty 0$ & 1.52 \\
\hline NaNO3 & ZERO & $8.246 E-02$ & ZERO & $8.246 \mathrm{E}-02$ & $1.504 \mathrm{E}-02$ & $6.743 \mathrm{E}-02$ & 1.52 \\
\hline $\mathrm{NaNO2}$ & ZERO & 1.270E-01 & ZERO & $1.270 \mathrm{E}-01$ & 2.316E-02 & 1.039E-01 & 1.52 \\
\hline $\mathrm{Na28407}$ & ZERO & $4.844 E-03$ & ZERO & $4.844 \mathrm{E}-03$ & $8.833 \mathrm{E}-04$ & $3.961 \mathrm{E}-03$ & 1.52 \\
\hline $\mathrm{Na} 2 \mathrm{SIO}_{3}$ & ZERO & $7.995 E-03$ & ZERO & $7.995 \mathrm{E}-03$ & $1.458 \mathrm{E}-03$ & $6.537 \mathrm{E}-03$ & 1.52 \\
\hline $\mathrm{N} \mid(\mathrm{NO} 3) 2$ & ZERO & $1.123 \mathrm{E}-01$ & ZERO & $1.123 \mathrm{E}-01$ & $2.048 E-02$ & $9.184 \mathrm{E}-02$ & 1.52 \\
\hline $\mathrm{Zn}(\mathrm{NO}) 2$ & ZERO & $7.685 \mathrm{E}-03$ & ZERO & 7.685E-03 & $1.401 \mathrm{E}-03$ & $6.284 \mathrm{E}-03$ & 1.52 \\
\hline $\mathrm{Zr}(\mathrm{NOO}) 4$ & ZERO & $1.892 \mathrm{E}-03$ & ZERO & $1.892 \mathrm{E}-03$ & $3.451 \mathrm{E}-04$ & $1.547 E-03$ & 1.52 \\
\hline \multicolumn{8}{|l|}{ Miscellaneous } \\
\hline Insoluble solids & ZERO & ZERO & ZERO & ZERO & ZERO & ZERO & \\
\hline HNO3 & ZERO & $9.142 E+01$ & ZERO & $9.142 E+01$ & $1.667 E+01$ & $7.475 E+01$ & \\
\hline $\mathrm{HCOOH}$ & ZERO & ZERO & ZERO & ZERO & ZERO & ZERO & \\
\hline \multicolumn{8}{|l|}{ Gases Water } \\
\hline $\mathrm{CO} 2$ & ZERO & ZERO & ZERO & ZERO & ZERO & ZERO & \\
\hline 02 & ZERO & ZERO & ZERO & ZERO & ZERO & ZERO & \\
\hline N2 & ZERO & ZERO & ZERO & ZERO & ZERO & ZERO & \\
\hline NOx & ZERO & ZERO & ZERO & ZERO & ZERO & ZERO & \\
\hline $\mathrm{H} 2 \mathrm{O}$ & $6.869 E+03$ & $8.381 E+03$ & ZERO & $8.381 \mathrm{E}+03$ & $1.528 E+03$ & $6.852 E+03$ & \\
\hline Nitric Acld (M) & ZERO & $1.560 \mathrm{E}-01$ & ZERO & 1.717E-01 & $1.107 \mathrm{E}-01$ & $1.717 \mathrm{E}-01$ & \\
\hline $\begin{array}{l}\text { Oxallc Acld (M) } \\
M n+2(M)\end{array}$ & \multicolumn{6}{|c|}{$\mathrm{Mn+2}(\mathrm{M})$} & \\
\hline Heat $(W)$ & & $3.512 \mathrm{E}+01$ & & $3.512 E+01$ & $3.511 E+01$ & $1.019 \mathrm{E}-02$ & \\
\hline$G(H 2)$ & & $1.074 E+00$ & & $1.062 E+00$ & $1.112 E+\infty 0$ & $1.062 E+\infty 0$ & \\
\hline H2 (g-mole/hr) & & $1.408 \mathrm{E}-02$ & & 1.391E-02 & $1.457 \mathrm{E}-02$ & $4.036 \mathrm{E}-06$ & \\
\hline Air Purge (SCFM) & & $1.793 \mathrm{E}-02$ & & $1.772 \mathrm{E}-02$ & $1.856 \mathrm{E}-02$ & 5.141E-06 & \\
\hline Volume (I) & $6.910 \mathrm{E}+00$ & $9.299 \mathrm{E}+\infty 0$ & $8.477 \mathrm{E}-01$ & $8.451 E+00$ & $2.389 E+00$ & $6.910 E+00$ & \\
\hline Mass (kg) & $6.931 E+00$ & $9.547 E+00$ & $9.546 E-01$ & $8.531 E+\infty 0$ & $2.510 E+00$ & $6.975 E+\infty 0$ & \\
\hline Sollds (g) & ZERO & $9.698 \mathrm{E}+02$ & $9.546 \mathrm{E}+02^{n}$ & 1.513E+01 & $9.574 E+02$ & 1.237E+01 & \\
\hline Sollds $(g /)$ & ZERO & $1.043 E+02$ & $1.126 \mathrm{E}+03$ & $1.790 \mathrm{E}+00$ & $4.008 E+02$ & $1.790 \mathrm{E}+00$ & \\
\hline \multirow{3}{*}{ Phase } & $1.003 \mathrm{E}+00$ & $1.027 E+00$ & $1.126 \mathrm{E}+\infty 0$ & $1.009 E+00$ & $1.051 E+00$ & $1.009 E+00$ & \\
\hline & Liquid & Liquid & Solid & Llquld & Liquid & Llquld & \\
\hline & & $\begin{array}{l}\text { oles Precip } \\
1.695 \mathrm{E}+00\end{array}$ & & & $\begin{array}{l}\text { ume Ratio } \\
1.823 E-01\end{array}$ & & \\
\hline
\end{tabular}


WSRC-TR-2000-00218

Revision 1

Page 33 of 42

Am/Cm Material Balance Spreadsheet Page 21

Version 2

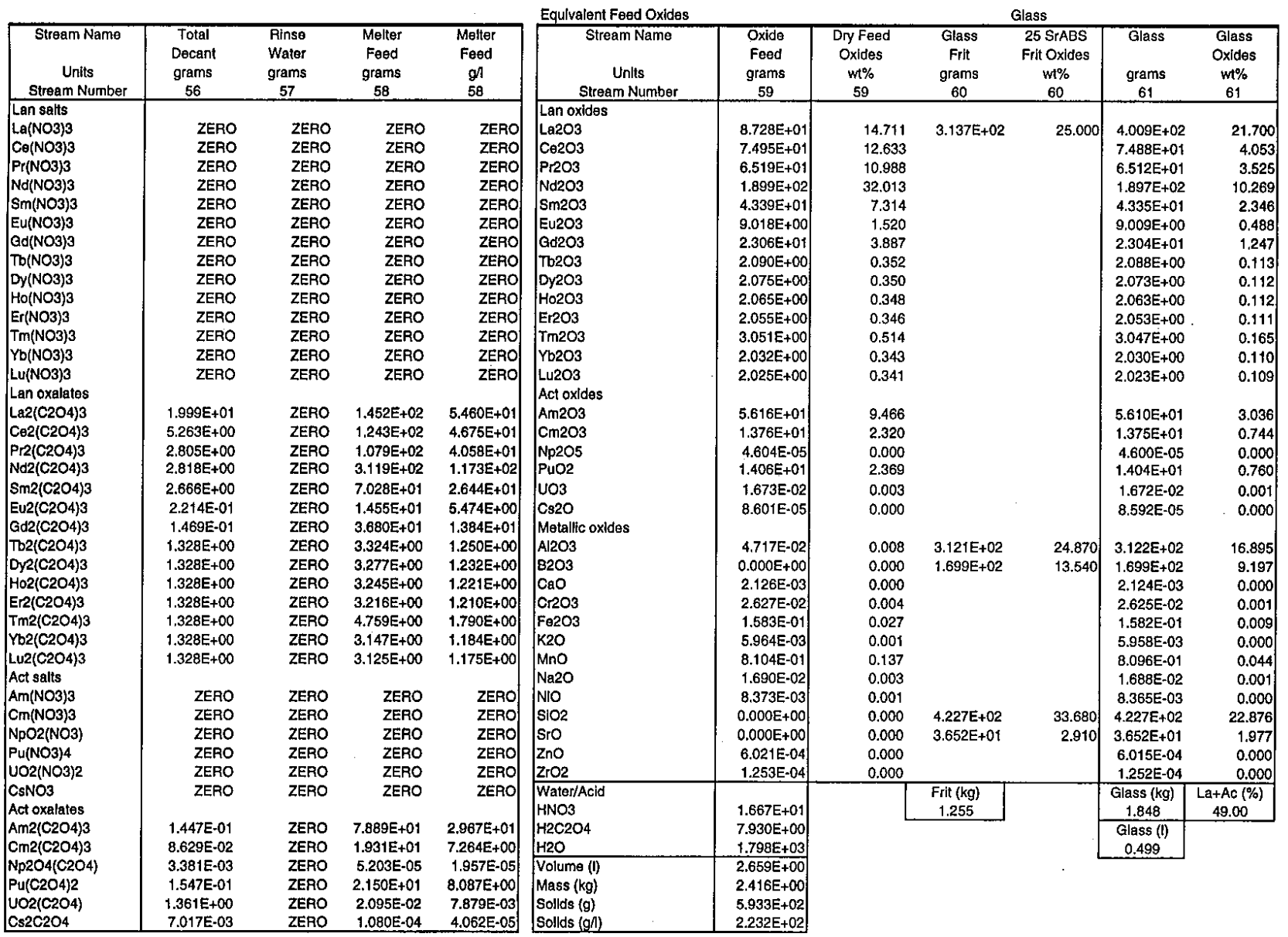


WSRC-TR-2000-00218

Revision 1

Page 34 of 42

Am/Cm Material Balance Spreadsheet Page 22

\begin{tabular}{|c|c|c|c|c|}
\hline $\begin{array}{c}\text { Stream Name } \\
\text { Unlts } \\
\text { Streem Number }\end{array}$ & $\begin{array}{l}\text { Total } \\
\text { Decant } \\
\text { grams } \\
56 \\
\end{array}$ & $\begin{array}{c}\text { Rlnse } \\
\text { Water } \\
\text { grams } \\
57 \\
\end{array}$ & $\begin{array}{c}\text { Melter } \\
\text { Feed } \\
\text { grams } \\
58\end{array}$ & $\begin{array}{c}\text { Melter } \\
\text { Feed } \\
g / 1 \\
58\end{array}$ \\
\hline \multicolumn{5}{|l|}{ Metallic salts } \\
\hline $\begin{array}{l}\mathrm{A} 22(\mathrm{SO}) 3) 3 \\
\mathrm{AlF3}\end{array}$ & $\begin{array}{r}6.171 E+00 \\
4.544 E-01\end{array}$ & $\begin{array}{l}\text { ZERO } \\
\text { ZERO }\end{array}$ & $\begin{array}{l}9.497 \mathrm{E}-02 \\
6.993 \mathrm{E}-03\end{array}$ & $\begin{array}{l}3.572 \mathrm{E}-02 \\
2.630 \mathrm{E}-03\end{array}$ \\
\hline Al(NO3)3 & $3.970 E+\infty 0$ & ZERO & $6.109 \mathrm{E}-02$ & 2.298E-02 \\
\hline $\mathrm{Ca}(\mathrm{NO}) 2$ & $4.042 E-01$ & ZERO & $6.221 E-03$ & $2.340 \mathrm{E}-03$ \\
\hline $\mathrm{Cr}(\mathrm{NO}) 3$ & $2.673 E+00$ & ZERO & 4.114E-02 & $1.548 \mathrm{E}-02$ \\
\hline $\mathrm{Fe} 2\left(\mathrm{SO}^{\prime}\right) 3$ & $1.247 E+01$ & ZERO & 1.919E-01 & $7.217 \mathrm{E}-02$ \\
\hline $\mathrm{FePO} 4$ & $2.8+8 E+00$ & ZERO & 4.336E-02 & $1.63 \uparrow \mathrm{E}-02$ \\
\hline $\mathrm{FeCl} 3$ & $2.194 E+00$ & ZERO & 3.377E-02 & $1.270 \mathrm{E}-02$ \\
\hline $\mathrm{Fe} 2(\mathrm{C} 2 \mathrm{O} 4) 3$ & $2.421 E+00$ & ZERO & $3.725 \mathrm{E}-02$ & $1.401 \mathrm{E}-02$ \\
\hline $\mathrm{Fe}(\mathrm{NO}) 3$ & $5.173 E+00$ & ZERO & $7.961 E-02$ & $2.994 \mathrm{E}-02$ \\
\hline KNO3 & $8.318 \mathrm{E}-01$ & ZERO & $1.280 \mathrm{E} \cdot 02$ & $4.815 \mathrm{E}-03$ \\
\hline $\mathrm{Mn}(\mathrm{NO}) 2$ & $1.328 E+02$ & ZERO & $2.044 E+\infty 0$ & $7.600 \mathrm{E}-01$ \\
\hline $\mathrm{NaNO}_{3}$ & $9.770 E-01$ & ZERO & $1.504 E-02$ & $5.655 \mathrm{E}-03$ \\
\hline NaNO2 & $1.505 \mathrm{E}+00$ & ZERO & 2.316E-02 & 8.713E-03 \\
\hline $\mathrm{Na2B} 8 \mathrm{O} 7$ & $5.739 \mathrm{E}-02$ & ZERO & 8.833E-04 & $3.322 \mathrm{E}-0$ \\
\hline $\mathrm{Na2SIO3}$ & $9.472 E-02$ & ZERO & $1.458 \mathrm{E}-03$ & 5.483E-04 \\
\hline Ni(NO3)2 & $1.331 E+\infty 0$ & ZERO & 2.048E-02 & $7.703 \mathrm{E}-03$ \\
\hline $\mathrm{Zn}(\mathrm{NO} 3)_{2}$ & $9.105 \mathrm{E}-02$ & ZERO & $1.401 \mathrm{E}-03$ & $5.271 \mathrm{E}-0$ \\
\hline $\mathrm{Zr}(\mathrm{NOO}) 4$ & $2.242 \mathrm{E}-02$ & ZERO & $3.451 E-04$ & $1.298 \mathrm{E}-0$ \\
\hline \multicolumn{5}{|l|}{ Mlscellaneous } \\
\hline insoluble solids & ZERO & ZERO & ZERO & ZERO \\
\hline HNO3 & $1.083 E+03$ & ZERO & $1.667 E+01$ & $6.270 E+0$ \\
\hline $\mathrm{HCOOH}$ & ZERO & ZERO & ZERO & ZERC \\
\hline $\mathrm{H} 2 \mathrm{C}_{2} \mathrm{O} 4$ & $5.153 \mathrm{E}+02$ & ZERO & $7.930 E+00$ & $2.983 E+00$ \\
\hline \multicolumn{5}{|l|}{ GasesWater } \\
\hline $\mathrm{CO} 2$ & ZERO & ZERO & ZERO & ZERO \\
\hline 02 & ZERO & ZERO & ZERO & ZERO \\
\hline N2 & ZERO & ZERO & ZERO & ZERO \\
\hline NOx & ZERO & ZERO & ZERO & ZERO \\
\hline $\mathrm{H} 2 \mathrm{O}$ & $2.353 E+04$ & $2,700 E+02$ & $1.798 E+03$ & $6.763 E+02$ \\
\hline Nitric Acld (M) & $7.328 \mathrm{E}-01$ & ZERO & $9.950 \mathrm{E}-02$ & \\
\hline Oxalle Acld (M) & $2.440 \mathrm{E}-01$ & ZERO & 3.313E-02 & \\
\hline \multicolumn{5}{|l|}{$M n+2(M)$} \\
\hline Heat $(M)$ & $1.568 \mathrm{E}-01$ & & $3.511 E+01$ & \\
\hline & 7.496E-01 & & $1.122 E+00$ & \\
\hline H2 (g-mole/hr) & 4.386E-05 & & $1.470 \mathrm{E}-02$ & \\
\hline Air Purgo (SCFM) & $5.586 \mathrm{E}-05$ & & $1.872 \mathrm{E}-02$ & \\
\hline Volume (l) & $2.345 E+01$ & $2.700 \mathrm{E}-01$ & $2.659 \mathrm{E}+00$ & \\
\hline Mass (kg) & $2.535 E+01$ & $2.700 \mathrm{E}-01$ & $2.780 E+\infty 0$ & \\
\hline Sollds (g) & $2.215 E+02$ & ZERO & $9.574 \mathrm{E}+02$ & \\
\hline Sollds (g/) & $9.442 \mathrm{E}+00$ & ZERO & $3.601 E+02$ & \\
\hline Denslty (g/ml) & $1.081 E+00$ & $1.000 E+00$ & $1.046 \mathrm{E}+00$ & \\
\hline Phase & Liquid & Llquld & Liquid & Liquid \\
\hline
\end{tabular}


WSRC.TR-2000-00218

Revision 1

Page 35 of 42

Am/Cm Material Balance Spreadsheet Page 23

\begin{tabular}{|c|c|c|c|c|c|c|c|c|}
\hline \multicolumn{2}{|l|}{ Melter Otf-Gas } & \multicolumn{7}{|l|}{ Ott-Gas Flow } \\
\hline $\begin{array}{c}\text { Stream Name } \\
\text { Unlts } \\
\text { Stream Number }\end{array}$ & $\begin{array}{c}\text { Oxide } \\
\text { Entrainment } \\
\text { Grams } \\
62 \\
\end{array}$ & \begin{tabular}{|c|} 
Stream Name \\
Unlts \\
Stream Number
\end{tabular} & $\begin{array}{c}\text { Sweep Air } \\
\begin{array}{c}9 / \mathrm{hr} \\
63\end{array} \\
\end{array}$ & $\begin{array}{l}\text { Combined } \\
\text { Off-Gas } \\
g / h r \\
64 \\
\end{array}$ & $\begin{array}{l}\text { Dilution Air } \\
\qquad \begin{array}{c}g / h r \\
65\end{array} \\
\end{array}$ & $\begin{array}{c}\text { Combined } \\
\text { Ott-Gas } \\
9 / \mathrm{hr} \\
66 \\
\end{array}$ & $\begin{array}{l}\text { Motive Air } \\
\begin{array}{c}\text { g/hr } \\
67\end{array}\end{array}$ & $\begin{array}{c}\text { Combined } \\
\text { Off-Gas } \\
g / h r \\
68 \\
\end{array}$ \\
\hline Lan oxides & & Lan oxldes & & & & & & \\
\hline $\begin{array}{l}\mathrm{La} 2 \mathrm{O} 3 \\
\mathrm{Ce} 2 \mathrm{O} 3\end{array}$ & $\begin{array}{l}8.728 \mathrm{E}-02 \\
7.495 \mathrm{E}-02\end{array}$ & $\begin{array}{l}\mathrm{La2O3} \\
\mathrm{Ce}_{\mathrm{B} 2 \mathrm{O}}\end{array}$ & $\begin{array}{l}\text { ZERO } \\
\text { ZERO }\end{array}$ & $\begin{array}{l}8.728 \mathrm{E}-02 \\
7.495 \mathrm{E}-02\end{array}$ & $\begin{array}{l}\text { ZERO } \\
\text { ZERO }\end{array}$ & $\begin{array}{l}8.728 \mathrm{E}-02 \\
7.495 \mathrm{E}-02\end{array}$ & $\begin{array}{l}\text { ZERO } \\
\text { ZERO }\end{array}$ & $\begin{array}{l}8.728 \mathrm{E}-02 \\
7.495 \mathrm{E}-02\end{array}$ \\
\hline $\begin{array}{l}\mathrm{Ce2O3} \\
\mathrm{Pr2O3}\end{array}$ & $\begin{array}{l}7.495 \mathrm{E}-02 \\
6.519 \mathrm{E}-02\end{array}$ & $\begin{array}{l}\mathrm{Ce} 2 \mathrm{O} 3 \\
\mathrm{Pr2O3}\end{array}$ & $\begin{array}{l}\text { ZERO } \\
\text { ZERO }\end{array}$ & $\begin{array}{l}7.495 \mathrm{E}-02 \\
6.519 \mathrm{E}-02\end{array}$ & $\begin{array}{l}\text { ZERO } \\
\text { ZERO }\end{array}$ & $\begin{array}{l}7.495 \mathrm{E}-02 \\
6.519 \mathrm{E}-02\end{array}$ & $\begin{array}{l}\text { ZERO } \\
\text { ZERO }\end{array}$ & $\begin{array}{l}7.495 \mathrm{E}-02 \\
6.519 \mathrm{E}-02\end{array}$ \\
\hline $\mathrm{Nd2O} 3$ & 1.899E-01 & $\mathrm{Nd2O} 3$ & ZERO & $1.899 \mathrm{E}-01$ & ZERO & $1.899 \mathrm{E}-01$ & ZERO & 1.899E-01 \\
\hline $\mathrm{Sm} 203$ & $4.339 \mathrm{E}-02$ & Sm2O3 & ZERO & $4.339 \mathrm{E}-02$ & ZERO & $4.339 \mathrm{E}-02$ & ZERO & $4.339 \mathrm{E}-02$ \\
\hline Eu2O3 & $9.018 \mathrm{E}-03$ & $\mathrm{Eu} 2 \mathrm{O} 3$ & ZERO & $9.018 \mathrm{E}-03$ & ZERO & $9.018 \mathrm{E}-03$ & ZERO & $9.018 \mathrm{E} \cdot 03$ \\
\hline Qd2O3 & $2.306 \mathrm{E}-02$ & Gd2O3 & ZERO & $2.306 \mathrm{E}-02$ & ZERO & $2.306 \mathrm{E}-02$ & ZERO & $2.306 \mathrm{E}-02$ \\
\hline Tb2O3 & $2.090 \mathrm{E}-03$ & $\mathrm{~Tb} 2 \mathrm{O} 3$ & ZERO & $2.090 \mathrm{E}-03$ & ZERO & $2,090 \mathrm{E}-03$ & ZERO & 2.090E-03 \\
\hline Dy2O3 & $2.075 \mathrm{E}-03$ & Dy203 & ZERO & 2.075E-03 & ZERO & $2.075 \mathrm{E}-03$ & ZERO & $2.075 \mathrm{E}-03$ \\
\hline $\mathrm{HO} 2 \mathrm{O} 3$ & $2.065 \mathrm{E}-03$ & $\mathrm{HO2O3}$ & ZERO & $2.065 \mathrm{E}-03$ & ZERO & $2.065 \mathrm{E}-03$ & ZERO & $2.065 \mathrm{E}-03$ \\
\hline Er2O3 & $2.055 \mathrm{E}-03$ & Er2O3 & ZERO & $2.055 E-03$ & ZERO & $2.055 \mathrm{E}-03$ & ZERO & $2.055 \mathrm{E}-03$ \\
\hline $\operatorname{Tm} 203$ & $3.051 E-03$ & Tm2O3 & ZERO & $3.051 E-03$ & ZERO & $3.051 \mathrm{E}-03$ & ZERO & $3.051 \mathrm{E}-03$ \\
\hline Yb2O3 & $2.032 \mathrm{E}-03$ & $\mathrm{Yb} 2 \mathrm{O} 3$ & ZERO & $2.032 \mathrm{E}-03$ & ZERO & $2.032 \mathrm{E}-03$ & ZERO & $2.032 \mathrm{E}-03$ \\
\hline Lu2O3 & $2.025 \mathrm{E}-03$ & Lu2O3 & ZERO & $2.025 \mathrm{E}-03$ & ZERO & $2.025 \mathrm{E}-03$ & ZERO & $2.025 \mathrm{E}-03$ \\
\hline Act oxides & & Act oxides & & & & & & \\
\hline Ann203 & $5.616 \mathrm{E}-02$ & Am2O3 & ZERO & $5.616 \mathrm{E}-02$ & ZERO & $5.616 \mathrm{E}-02$ & ZERO & $5.616 \mathrm{E}-02$ \\
\hline $\mathrm{Cm} 2 \mathrm{O3}$ & $1.376 \mathrm{E}-02$ & $\mathrm{Cm} 2 \mathrm{O} 3$ & ZERO & $1.376 \mathrm{E}-02$ & ZERO & $1.376 \mathrm{E}-02$ & ZERO & $1.376 \mathrm{E}-02$ \\
\hline $\mathrm{Np2O5}$ & 4.604E-08 & $\mathrm{Np2O5}$ & ZERO & 4.604E-08 & ZERO & 4.604E-OB & ZERO & $4.604 \mathrm{E}-08$ \\
\hline PuO2 & $1.406 \mathrm{E} \cdot 02$ & PuO2 & ZERO & $1.406 \mathrm{E}-02$ & ZERO & $1.406 \mathrm{E}-02$ & ZERO & $1.406 \mathrm{E}-02$ \\
\hline vo3 & $1.673 \mathrm{E} \cdot 05$ & uo3 & ZERO & $1.673 \mathrm{E}-05$ & ZERO & $1.673 \mathrm{E}-05$ & ZERO & $1.673 \mathrm{E}-05$ \\
\hline Cs2O & $8.601 E-08$ & Cs2O & ZERO & $8.601 E-08$ & ZERO & $8.601 E-08$ & ZERO & $8.601 \mathrm{E}-08$ \\
\hline Metallle oxides & & Metalllc oxides & & & & & & \\
\hline Al2O3 & $4.717 \mathrm{E} \cdot 05$ & Al2O3 & ZERO & 4.717E-05 & ZERO & 4.717E-05 & ZERO & 4.717E-05 \\
\hline $\mathrm{B2O3}$ & $0.000 E+00$ & 8203 & ZERO & $0.000 E+00$ & ZERO & $0.000 E+00$ & ZERO & $0.000 \mathrm{E}+00$ \\
\hline $\mathrm{CaO}$ & $2.126 \mathrm{E}-06$ & $\mathrm{CaO}$ & ZERO & $2.126 \mathrm{E}-06$ & ZERO & $2.126 \mathrm{E}-06$ & ZERO & $2.126 \mathrm{E}-06$ \\
\hline $\mathrm{Cr} 2 \mathrm{O} 3$ & 2.627E-05 & $\mathrm{Cr} 2 \mathrm{O} 3$ & ZERO & 2.627E-05 & ZERO & $2.627 \mathrm{E}-05$ & ZERO & $2.627 \mathrm{E}-05$ \\
\hline $\mathrm{F} \theta 2 \mathrm{O3}$ & $1.583 \mathrm{E} \cdot 04$ & $\mathrm{Fe} 2 \mathrm{O} 3$ & ZERO & $1.583 \mathrm{E}-04$ & ZERO & $1.583 \mathrm{E}-04$ & ZERO & $1.583 \mathrm{E}-04$ \\
\hline K2O & $5.964 \mathrm{E} \cdot 06$ & K2O & ZERO & $5.964 \mathrm{E}-06$ & ZERO & $5.964 \mathrm{E}-06$ & ZERO & $5.964 \mathrm{E}-06$ \\
\hline MnO & $8.104 \mathrm{E}-04$ & $\mathrm{MnO}$ & ZERO & $8.104 \mathrm{E}-04$ & ZERO & $8.104 E-04$ & ZERO & $8.104 E-04$ \\
\hline $\mathrm{Na} 2 \mathrm{O}$ & $1.690 \mathrm{E}-05$ & $\mathrm{Na} 2 \mathrm{O}$ & ZERO & $1.690 \mathrm{E}-05$ & ZERO & t.690E-05 & ZERO & $1.690 \mathrm{E}-05$ \\
\hline NIO & $8.373 E-06$ & $\mathrm{NiO}$ & ZERO & 8.373E-06 & ZERO & $8.373 E-06$ & ZERO & $8.373 \mathrm{E}-06$ \\
\hline $\mathrm{SIO2}$ & $0.000 \mathrm{E}+00$ & $\mathrm{SiO} 2$ & ZERO & $0.000 \mathrm{E}+00$ & ZERO & $0.000 E+00$ & ZERO & $0.000 E+00$ \\
\hline Sro & $0.000 E+00$ & Sro & ZERO & $0.000 \mathrm{E}+00$ & ZERO & $0.000 E+00$ & ZERO & $0.000 E+00$ \\
\hline Zno & $6.021 \mathrm{E}-07$ & Zno & ZERO & $6.021 \mathrm{E}-07$ & ZERO & $6.021 \mathrm{E}-07$ & ZERO & $6.021 \mathrm{E}-07$ \\
\hline $\mathrm{ZrO2}$ & $1.253 E-07$ & $\mathrm{ZrO} 2$ & ZERO & $1.253 \mathrm{E}-07$ & ZERO & $1.253 \mathrm{E}-07$ & ZERO & $1.253 \mathrm{E}-07$ \\
\hline Total Mass (g) & $5.933 \mathrm{E}-01$ & & & & & & & \\
\hline
\end{tabular}


WSRC-TR-2000-00218

Revision 1

Page 36 of 42

Am/Cm Material Balance Spreadsheet Page 24

Version 2

('lhis Page Intentionally Left Blank) 
WSRC-TR-2000-00218

Revision 1

Page 37 of 42

Am/Cm Material Balance Spreadsheet Page 25

Version 2

\begin{tabular}{|c|c|}
\hline Melter Off-Gas & Drying \\
\hline $\begin{array}{c}\text { Stream Name } \\
\text { Units } \\
\text { Stream Number }\end{array}$ & $\begin{array}{c}\text { Reaction } \\
\text { Products } \\
\text { grams } \\
62 \\
\end{array}$ \\
\hline $\begin{array}{l}\text { Gases } \\
\mathrm{H} 2 \mathrm{O} \\
\mathrm{CO} \\
\mathrm{CO} 2 \\
\mathrm{O} 2 \\
\mathrm{~N} 2 \\
\mathrm{NOx}(\mathrm{NO}+\mathrm{NO} 2) / 2 \\
\mathrm{SO} 3 \\
\mathrm{POx}(\mathrm{PO} 2+\mathrm{PO} 3) / 2 \\
\mathrm{HCl} \\
\mathrm{HF} \\
\end{array}$ & $1.798 \mathrm{E}+03$ \\
\hline $\begin{array}{l}\text { Temperature (C) } \\
\text { Pressure (in. } \mathrm{H} 2 \mathrm{O} \text { ) } \\
\text { Volume (l) } \\
\text { Mass (g) } \\
\text { Sollds }(\mathrm{g}) \\
\text { Sollds }(\mathrm{g} /) \\
\text { Density }(g /) \\
\text { Phase }\end{array}$ & $\begin{array}{r}200.00 \\
-2.00 \\
3.894 \mathrm{E}+03 \\
1.798 \mathrm{E}+03 \\
5.933 \mathrm{E}-01 \\
1.524 \mathrm{E}-04 \\
4.619 \mathrm{E}-01 \\
\text { Vapor }\end{array}$ \\
\hline
\end{tabular}

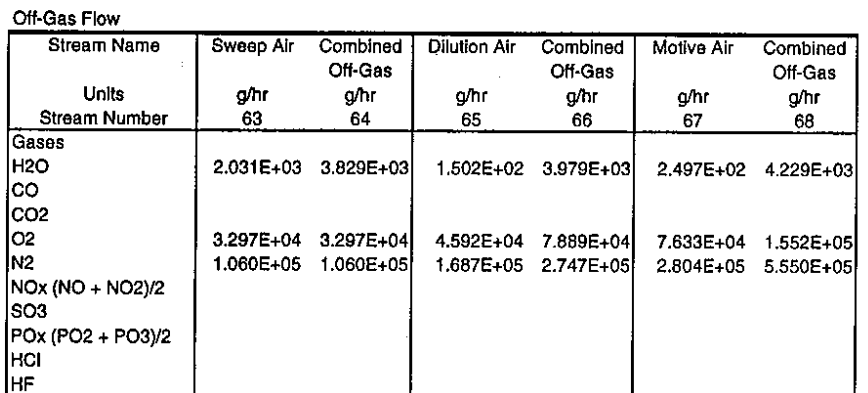

\begin{tabular}{|c|c|c|c|c|c|c|}
\hline Temperature $(\mathrm{C})$ & 20.00 & 24.26 & 20.00 & 21.70 & 20.00 & 20.85 \\
\hline Pressure (in. $\mathrm{H} 2 \mathrm{O}$ ) & 0.00 & 0.00 & 0.00 & 0.00 & 0.00 & 0.00 \\
\hline Volume Flow (Vht) & $1.185 \mathrm{E}+05$ & $1.227 \mathrm{E}+05$ & $1.790 \mathrm{E}+05$ & $3.022 \mathrm{E}+05$ & $2.975 \mathrm{E}+05$ & $6.006 \mathrm{E}+05$ \\
\hline Mass Flow $(g / h r)$ & $1.410 \mathrm{E}+05$ & $1.428 E+b 5$ & $2.146 E+05$ & $3.575 \mathrm{E}+05$ & $3.567 \mathrm{E}+05$ & $7.145 E+05$ \\
\hline Solids Flow (g/hr) & & 5.933E-01 & & $5.933 E-01$ & & $5.933 \mathrm{E}-01$ \\
\hline Solids $(g /)$ & & $4.836 \mathrm{E}-06$ & & $1.963 E-06$ & & $9.878 E-07$ \\
\hline Density $(g)$ & $1.190 \mathrm{E}+00$ & $1.164 E+00$ & $1.199 \mathrm{E}+00$ & $1.183 E+00$ & $1.199 E+00$ & $1,190 E+00$ \\
\hline Phase & Vapor & Vapor & Vapor & Vapor & Vapor & Vapor \\
\hline Flow (SCFM), & $6.500 \mathrm{E}+01$ & $6.633 \mathrm{E}+01$ & $9.815 E+01$ & $1.648 E+02$ & $1.632 E+02$ & $3.285 E+02$ \\
\hline Temperature (K) & $2.932 \mathrm{E}+02$ & $2.974 E+02$ & $2.932 E+02$ & $2.949 \mathrm{E}+02$ & $2.932 \mathrm{E}+02$ & $2.940 \mathrm{E}+02$ \\
\hline Molecular Welght & $2.861 E+01$ & $2.840 E+01$ & $2.884 E+01$ & $2.866 \mathrm{E}+01$ & $2.884 E+01$ & $2.875 E+01$ \\
\hline Pvap (atm) & 2.288E-02 & $4.242 \mathrm{E}-02$ & $1.125 \mathrm{E}-03$ & $1.777 \mathrm{E}-02$ & $1.125 \mathrm{E}-03$ & $9.483 \mathrm{E}-03$ \\
\hline Psat (atm) & $2.288 \mathrm{E}-02$ & $2.963 \mathrm{E}-02$ & 2.288E-02 & $2.539 \mathrm{E}-02$ & $2,288 \mathrm{E}-02$ & $2.411 \mathrm{E}-02$ \\
\hline Dow Point (C) & $1.992 E+01$ & $3.027 \mathrm{E}+01$ & $-2.080 E+01$ & $1.592 E+01$ & $-2.080 E+01$ & $6.488 E+00$ \\
\hline Humldity (\%) & $1.000 E+02$ & $1.432 E+02$ & $4.918 E+00$ & $7,000 E+01$ & $4.918 E+00$ & $3.934 \mathrm{E}+01$ \\
\hline$\Delta \mathrm{T}(\mathrm{C})$ & & $-6.015 \mathrm{E}+00$ & & $5.781 \mathrm{E}+00$ & & $1.436 \mathrm{E}+01$ \\
\hline
\end{tabular}


WSRC-TR-2000-00218

Revision 1

Page 38 of 42

Am/Cm Material Balance Spreadsheet Page 26

Version 2

\begin{tabular}{|c|c|}
\hline Melter Off-Gas & Drying \\
\hline $\begin{array}{c}\text { Stream Name } \\
\text { Units } \\
\text { Stream Number }\end{array}$ & $\begin{array}{c}\text { Reaction } \\
\text { Products } \\
\text { grams } \\
62\end{array}$ \\
\hline $\begin{array}{l}\text { Gases } \\
\mathrm{H} 2 \mathrm{O} \\
\mathrm{CO} \\
\mathrm{CO} 2 \\
\mathrm{O} 2 \\
\mathrm{~N} 2 \\
\mathrm{NO} \times(\mathrm{NO}+\mathrm{NO} 2) / 2 \\
\mathrm{SO} 3 \\
\mathrm{POx}(\mathrm{PO} 2+\mathrm{PO} 3) / 2 \\
\mathrm{HCl} \\
\mathrm{HF}\end{array}$ & $1.798 E+03$ \\
\hline
\end{tabular}

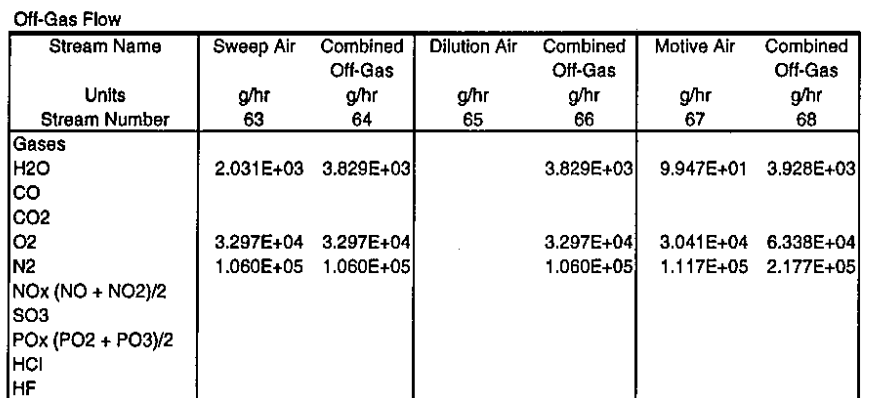

\begin{tabular}{|l|r|}
\hline Tempersture (C) & 200.00 \\
Pressure (in. H2O) & -2.00 \\
Volume (l) & $3.894 \mathrm{E}+03$ \\
Mass (g) & $1.798 \mathrm{E}+03$ \\
Sollds (g) & $5.933 \mathrm{E}-01$ \\
Solids (g/) & $1.524 \mathrm{E}-04$ \\
Density (g/) & $4.619 \mathrm{E}-01$ \\
\hline Phase & Vapor \\
\hline
\end{tabular}

\begin{tabular}{|c|c|c|c|c|c|c|}
\hline Temperature $(\mathrm{C})$ & 20.00 & 24.26 & & \begin{tabular}{|l|}
36.73 \\
\end{tabular} & 20.00 & 28.39 \\
\hline Pressure (in. $\mathrm{H} 2 \mathrm{O}$ ) & 0.00 & 0.00 & & 0.00 & 0.00 & 0.00 \\
\hline Volume Flow (Vhr) & $1.185 E+05$ & $1.227 \mathrm{E}+05$ & & $1.278 E+05$ & 1.185E+05 & $2.467 E+05$ \\
\hline Mass Flow (ghr) & $1.410 E+05$ & $1.428 E+05$ & & $1.428 E+05$ & $1.421 \mathrm{E}+05$ & $2.850 \mathrm{E}+05$ \\
\hline Solids Flow $(g / h r)$ & & $5.933 \mathrm{E}-01$ & & 5.933E-01 & & $5.933 E-01$ \\
\hline Solids $(g /)$ & & $4.836 \mathrm{E}-06$ & & 4.641E-06 & & $2.405 \mathrm{E}-06$ \\
\hline Density $(g)$ & $1.190 E+00$ & $1.164 \mathrm{E}+\infty$ & & $1.117 E+00$ & $1.199 \mathrm{E}+00$ & $1.155 E+00$ \\
\hline \multirow[t]{2}{*}{$\begin{array}{l}\text { Phase } \\
\end{array}$} & Vapor & Vapor & Vapor & Vapor & Vapor & Vapor \\
\hline & & & & & & \\
\hline Flow (SCFM) & $6.500 E+01$ & $6.633 E+01$ & & 6.633E+01 & $6.500 \mathrm{E}+01$ & $1.315 \mathrm{E}+02$ \\
\hline Temperature (K) & $2.932 E+02$ & $2.974 E+02$ & & $3.099 \mathrm{E}+02$ & $2.932 \mathrm{E}+02$ & $3.015 E+02$ \\
\hline Molecular Weight & $2.861 E+01$ & $2.840 \mathrm{E}+01$ & & $2.840 E+01$ & $2.884 E+01$ & $2.862 E+01$ \\
\hline Pvap (atm) & $2.288 \mathrm{E}-02$ & 4.242E-02 & & 4.242E-02 & $1.125 \mathrm{E}-03$ & $2.198 \mathrm{E}-02$ \\
\hline Psat (atm) & $2.288 \mathrm{E}-02$ & 2.963E-02 & & $6.060 E-02$ & $2.288 \mathrm{E}-02$ & $3.781 \mathrm{E}-02$ \\
\hline Dew Point (C) & $1.992 E+01$ & $3.027 E+01$ & & $3.027 \mathrm{E}+01$ & $-2.080 E+01$ & $1.928 E+01$ \\
\hline Humidlty $(\%)$ & $1.000 E+02$ & $1.432 E+02$ & & $7.000 E+01$ & $4.918 E+00$ & $5.812 E+01$ \\
\hline$\Delta T(\bar{C})$ & & $-6.015 \mathrm{E}+00$ & & $6.461 E+00$ & & $9.111 E+00$ \\
\hline
\end{tabular}


WSRC-TR-2000-00218

Revision 1

Version 2

Page 40 of 42

Am/Cm Material Balance Spreadsheet Page 28

\begin{tabular}{|c|c|}
\hline Melter Off-Gas & Calcination \\
\hline $\begin{array}{c}\text { Stream Name } \\
\text { Units } \\
\text { Stream Number }\end{array}$ & $\begin{array}{c}\text { Reaction } \\
\text { Products } \\
\text { grams } \\
69\end{array}$ \\
\hline Gases & \\
\hline $\begin{array}{l}\mathrm{H} 2 \mathrm{O} \\
\mathrm{CO}\end{array}$ & $\begin{array}{l}3.962 E+00 \\
1.434 E+02\end{array}$ \\
\hline $\mathrm{CO} 2$ & $2.254 E+02$ \\
\hline N2 & $0.000 E+00$ \\
\hline $\mathrm{NO} \times(\mathrm{NO}+\mathrm{NO} 2) / 2$ & $1.105 \mathrm{E}+01$ \\
\hline $\mathrm{sO3}$ & $1.819 \mathrm{E}-01$ \\
\hline $\mathrm{PO}(\mathrm{PO} 2+\mathrm{PO} 3) / 2$ & $1.020 \mathrm{E}-02$ \\
\hline $\mathrm{HCl}$ & $2.277 \mathrm{E}-02$ \\
\hline $\mathrm{HF}$ & 4.998E- 03 \\
\hline Temperature $(\mathrm{C})$ & 700.00 \\
\hline Pressure (in. $\mathrm{H}_{2} \mathrm{O}$ ) & $\cdot 2.00$ \\
\hline Volume (1) & $8.656 \mathrm{E}+02$ \\
\hline Mass (g) & $3.851 \mathrm{E}+02$ \\
\hline Sollds (g) & \\
\hline Sollds $(g /)$ & \\
\hline Density $(g /)$ & $4.448 \mathrm{E}-01$ \\
\hline Phase & Vapor \\
\hline
\end{tabular}

\begin{tabular}{|c|c|c|c|c|c|c|}
\hline $\begin{array}{c}\text { Stream Name } \\
\text { Units } \\
\text { Stream Number }\end{array}$ & $\begin{array}{l}\text { Sweop Alr } \\
\qquad \begin{array}{c}9 / \mathrm{hr} \\
70\end{array}\end{array}$ & $\begin{array}{c}\text { Combined } \\
\text { Off-Gas } \\
\text { g/hr } \\
71\end{array}$ & $\begin{array}{l}\text { Dilution Air } \\
\qquad \begin{array}{c}9 / \mathrm{hr} \\
72\end{array}\end{array}$ & $\begin{array}{c}\text { Combined } \\
\text { Off-Gas } \\
g / h r \\
73\end{array}$ & $\begin{array}{l}\text { Motive Air } \\
\qquad \begin{array}{c}g / h r \\
74\end{array}\end{array}$ & $\begin{array}{c}\text { Combined } \\
\text { Off-Gas } \\
9 / h r \\
75\end{array}$ \\
\hline Gases & & & & & & \\
\hline $\begin{array}{l}\mathrm{H} 2 \mathrm{O} \\
\mathrm{CO}\end{array}$ & $2.031 E+03$ & $\begin{array}{l}2.035 E+03 \\
1.148 E+02\end{array}$ & & $\begin{array}{l}2.035 E+03 \\
1.148 E+02\end{array}$ & $9.947 E+01$ & $\begin{array}{l}2.134 E+03 \\
1.148 E+02\end{array}$ \\
\hline $\mathrm{CO} 2$ & & $2.705 \mathrm{E}+02$ & & $2.705 E+02$ & & $2.705 E+02$ \\
\hline $\mathrm{O} 2$ & $3.297 E+04$ & 3.297E+04 & & $3.297 \varepsilon+04$ & $3.041 E+04$ & $6.338 \mathrm{E}+04$ \\
\hline N2 & $1.060 E+05$ & $1.060 \mathrm{E}+05$ & & $1.060 \varepsilon+05$ & $1.117 E+05$ & 2.177E+05 \\
\hline $\mathrm{NOx}(\mathrm{NO}+\mathrm{NO} 2) / 2$ & & $5.524 \mathrm{E}+00$ & & $5.524 E+00$ & & $5.524 E+00$ \\
\hline $\mathrm{SO}_{3}$ & & $1.819 \mathrm{E}-01$ & & $1.819 \mathrm{E}-01$ & & $1.819 E-01$ \\
\hline $\mathrm{POX}(\mathrm{PO} 2+\mathrm{PO} 3) / 2$ & & $1.020 \mathrm{E}-02$ & & $1.020 \mathrm{E}-02$ & & $1.020 \mathrm{E}-02$ \\
\hline $\mathrm{HCl}$ & & $2.277 \mathrm{E}-02$ & & $2.277 \mathrm{E}-02$ & & 2.277E-02 \\
\hline $\mathrm{HF}$ & & $4.998 \mathrm{E}-03$ & & 4.998E-03 & & 4.998E-03 \\
\hline Temperature $(\mathrm{C})$ & 20.00 & 21.85 & & 25.97 & 20.00 & 22.98 \\
\hline Pressure (In. $\mathrm{H} 2 \mathrm{O})$ & 0.00 & 0.00 & & 0.00 & 0.00 & 0.00 \\
\hline Volume flow ( $(\mathrm{hr})$ & $1.185 E+05$ & $1.195 E+05$ & & $1.212 E+05$ & $1.185 E+05$ & $2.401 E+05$ \\
\hline Mass Flow $(g / h r)$ & $1.410 \mathrm{E}+05$ & 1.414E+05 & & $1.414 E+05$ & $1.421 E+05$ & $2.836 E+05$ \\
\hline Solds Flow (g/hr) & & & & & & \\
\hline Solds $(91)$ & & & & & & \\
\hline Density $(g /)$ & $1.190 E+00$ & $1.183 E+00$ & & $1.167 E+\infty 0$ & $1.199 \mathrm{E}+00$ & $1.181 \mathrm{E}+00$ \\
\hline Phase & Vapor & Vapor & Vapor & Vapor & Vapor & Vapor \\
\hline Flow (SCFM) & $6.500 E+01$ & $6.515 E+01$ & & $6.515 \mathrm{E}+01$ & $6.500 \mathrm{E}+01$ & $1.304 E+02$ \\
\hline Temperature $(K)$ & $2.932 E+02$ & $2.950 \mathrm{E}+02$ & & $2,991 \mathrm{E}+02$ & $2,932 E+02$ & $2.961 \mathrm{E}+02$ \\
\hline Molecular Wolght & $2.861 E+01$ & $2.861 E+01$ & & $2.861 E+01$ & $2.884 E+01$ & $2.873 \mathrm{E}+01$ \\
\hline Pvap (atm) & 2.288E-02 & 2.294E-02 & & 2.294E-02 & $1.125 \mathrm{E}-03$ & 1.204E-02 \\
\hline Psat (atm) & $2.288 \mathrm{E}-02$ & $2.563 \mathrm{E}-02$ & & $3.280 \mathrm{E}-02$ & $2.288 E-02$ & $2.743 \mathrm{E}-02$ \\
\hline Dow Point (C) & $1.992 \mathrm{E}+01$ & $1.996 \mathrm{E}+01$ & & $1.996 \mathrm{E}+01$ & $-2.080 E+01$ & $9.992 E+00$ \\
\hline Humldity $(\%)$ & $1.000 E+02$ & $8.951 E+01$ & & $6.992 \mathrm{E}+01$ & $4.918 \mathrm{E}+00$ & $4.391 E+01$ \\
\hline$\Delta \mathrm{T}(\mathrm{C})$ & & $1.890 \mathrm{E}+00$ & & $6.003 \vec{E}+00$ & & $1.298 \mathrm{E}+01$ \\
\hline
\end{tabular}


WSRC-TR-2000-00218

Revision 1

Page 41 of 42

Am/Cm Material Balance Spreadsheet Page 29

Version 2

\begin{tabular}{|c|c|}
\hline Melter Off-Qas & Viltrificatlon \\
\hline $\begin{array}{c}\text { Stream Name } \\
\text { Units } \\
\text { Stream Number }\end{array}$ & $\begin{array}{c}\text { Reaction } \\
\text { Products } \\
\text { grams } \\
76\end{array}$ \\
\hline $\begin{array}{l}\text { Gases } \\
\mathrm{H} 2 \mathrm{O} \\
\mathrm{CO} \\
\mathrm{CO} 2 \\
\mathrm{O} 2 \\
\mathrm{~N} 2 \\
\mathrm{NOx}(\mathrm{NO}+\mathrm{NO} 2) / 2 \\
\mathrm{SO} 3 \\
\mathrm{POx}(\mathrm{PO} 2+\mathrm{PO} 3) / 2 \\
\mathrm{HCl} \\
\mathrm{HF}\end{array}$ & $3.653 E+00$ \\
\hline \begin{tabular}{|l|} 
Temperature (C) \\
Pressure (in. H2O) \\
Volume (l) \\
Mass $(g)$ \\
Solids $(g)$ \\
Sollds $(g /)$ \\
Density (g/)
\end{tabular} & $\begin{array}{r}700.00 \\
-2.00 \\
9.162 E+00 \\
3.653 E+00\end{array}$ \\
\hline Phase & Vapor \\
\hline
\end{tabular}

\begin{tabular}{|c|c|c|c|c|c|c|}
\hline $\begin{array}{c}\text { Stream Name } \\
\text { Units } \\
\text { Stream Number }\end{array}$ & $\begin{array}{l}\text { Sweep Air } \\
\qquad \begin{array}{l}\text { ghr } \\
77\end{array}\end{array}$ & $\begin{array}{c}\text { Combined } \\
\text { Off-Gas } \\
\text { g/hr } \\
78\end{array}$ & $\begin{array}{l}\text { Dilution Air } \\
\qquad \begin{array}{l}\text { g/hr } \\
79\end{array}\end{array}$ & $\begin{array}{c}\text { Combined } \\
\text { Off-Gas } \\
g / h r \\
80\end{array}$ & $\begin{array}{l}\text { Motive Air } \\
\qquad \begin{array}{l}9 / \mathrm{hr} \\
81\end{array}\end{array}$ & $\begin{array}{l}\text { Combined } \\
\text { Off-Gas } \\
\text { g/hr } \\
82\end{array}$ \\
\hline \multicolumn{7}{|l|}{ Gases } \\
\hline $\begin{array}{l}\mathrm{H} 2 \mathrm{O} \\
\mathrm{CO}\end{array}$ & $2.031 E+03$ & $2.031 E+03$ & $4.626 E+01$ & $2.077 E+03$ & $1.457 \varepsilon+02$ & $2.223 \mathrm{E}+03$ \\
\hline $\mathrm{CO} 2$ & & & & & & \\
\hline 02 & $3.297 E+04$ & $3.297 E+04$ & $1.414 E+04$ & $4.712 \mathrm{E}+04$ & $4.455 \mathrm{E}+04$ & $9.167 \mathrm{E}+04$ \\
\hline N2 & $1.060 E+05$ & $1.060 \mathrm{E}+05$ & $5.195 \mathrm{E}+04$ & $1.580 \mathrm{E}+05$ & $1.636 \mathrm{E}+05$ & $3.216 E+05$ \\
\hline \multirow{4}{*}{\multicolumn{7}{|c|}{$\begin{array}{l}\mathrm{NOx}(\mathrm{NO}+\mathrm{NO} 2) / 2 \\
\text { SO3 } \\
\mathrm{POx}(\mathrm{PO} 2+\mathrm{PO}) / 2 \\
\mathrm{HCl} \\
\mathrm{HF}\end{array}$}} \\
\hline & & & & & & \\
\hline & & & & & & \\
\hline & & & & & & \\
\hline Temperature (C) & 20.00 & 20.02 & 20.00 & 20.01 & 20.00 & 20.01 \\
\hline Pressure (in. $\mathrm{H} 2 \mathrm{O}$ ) & 0.00 & 0.00 & 0.00 & 0.00 & 0.00 & 0.00 \\
\hline Volume Flow (/hr) & $1.185 E+05$ & $1.185 E+05$ & $5.512 E+04$ & $1.738 \mathrm{E}+05$ & $1.736 E+05$ & $3.480 E+05$ \\
\hline Mass Flow $(g / h r)$ & $1.410 \mathrm{E}+05$ & $1.410 \mathrm{E}+05$ & $6.609 \mathrm{E}+04$ & $2.072 E+05$ & $2.082 E+05$ & $4.155 E+05$ \\
\hline Sollds Flow $(g / h r)$ & & & & & & \\
\hline Sollds $(g /)$ & & & & & & \\
\hline Densily $(g /)$ & $1.190 \mathrm{E}+\infty 0$ & $1.190 \mathrm{E}+00$ & $1.199 E+00$ & $1.192 E+00$ & $1.199 \mathrm{E}+00$ & $1.194 \mathrm{E}+00$ \\
\hline Phase & Vapor & Vapor & Vapor & Vapor & Vapor & Vapor \\
\hline Flow (SCFM) & $6.500 E+01$ & $6.501 \mathrm{E}+01$ & $3.023 E+01$ & $9.534 \mathrm{E}+01$ & $9.523 \mathrm{E}+01$ & $1.909 E+02$ \\
\hline Temperature (K) & $2.932 E+02$ & $2.932 E+02$ & $2.932 E+02$ & $2.932 \mathrm{E}+02$ & $2.932 E+02$ & $2.932 E+02$ \\
\hline Molecular Weight & $2.861 E+01$ & $2.861 E+01$ & $2.884 E+01$ & $2.868 \mathrm{E}+01$ & $2.884 E+01$ & $2.876 \mathrm{E}+01$ \\
\hline Pvap (atm) & $2.288 \mathrm{E}-02$ & $2.296 \mathrm{E}-02$ & $1.125 \mathrm{E}-03$ & $1.602 E-02$ & $1.125 \mathrm{E}-03$ & $8.574 \mathrm{E}-03$ \\
\hline Psat (atm) & 2.288E-02 & $2.290 \mathrm{E}-02$ & $2.288 \mathrm{E}-02$ & $2,289 \mathrm{E}-02$ & $2.288 \mathrm{E}-02$ & $2.288 \mathrm{E}-02$ \\
\hline Dew Point (C) & $1.992 \mathrm{E}+01$ & $1.997 E+01$ & $-2.080 E+01$ & $1.431 E+01$ & $-2.080 E+01$ & $5.042 E+00$ \\
\hline Humidlty (\%) & $1.000 \mathrm{E}+02$ & $1.002 E+02$ & $4.918 \mathrm{E}+00$ & $7.000 E+01$ & $4.918 \mathrm{E}+00$ & $3.747 E+01$ \\
\hline$\Delta \mathrm{T}(\mathrm{C})$ & & $4.265 \mathrm{E}-02$ & & $5.699 \mathrm{E}+00$ & & $1.496 \mathrm{E}+01$ \\
\hline
\end{tabular}


WSRC-TR-2000-00218

Revision 1

Page 42 of 42

Am/Cm Material Balance Spreadsheet Page 30

Version 2

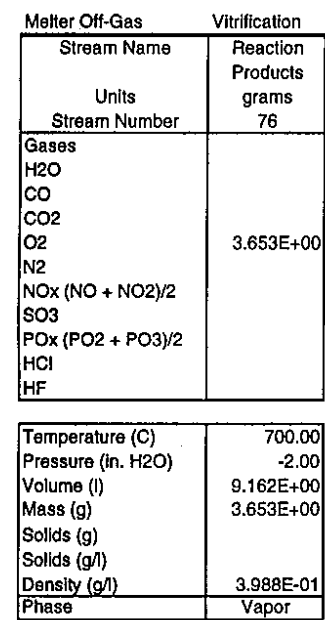

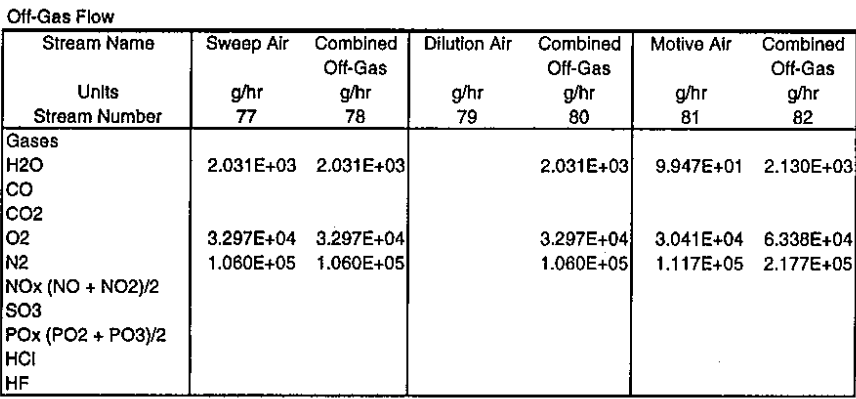

\begin{tabular}{|c|c|c|c|c|c|c|}
\hline Temperature (C) & 20.00 & 20.02 & & 25.96 & 20.00 & 22.97 \\
\hline Pressure (in. $\mathrm{H} 2 \mathrm{O}$ ) & 0.00 & 0.00 & & 0.00 & 0.00 & 0.00 \\
\hline Volume Flow (Vhr) & $1.185 E+05$ & $1.185 E+05$ & & $1.209 E+05$ & $1.185 E+05$ & $2.398 \mathrm{E}+05$ \\
\hline Mass Flow (g/hr) & $1.410 E+05$ & $1.410 E+05$ & & 1.410E+05 & $1.421 E+05$ & $2.832 \mathrm{E}+05$ \\
\hline \multicolumn{7}{|l|}{$\begin{array}{l}\text { Solids Flow }(g / h r) \\
\text { Solids }(g /)\end{array}$} \\
\hline Density $(g /)$ & $1.190 \mathrm{E}+00$ & $1.190 E+00$ & & $1.166 E+00$ & $1.199 E+00$ & $1.181 \mathrm{E}+00$ \\
\hline Phase & Vapor & Vapor & Vapor & Vapor & Vapor & Vapor \\
\hline Flow (SCFM) & $6.500 \mathrm{E}+01$ & $6.501 E+01$ & & $6.501 E+01$ & $6.500 E+01$ & $1.302 \mathrm{E}+02$ \\
\hline Temperature (K) & $2.932 E+02$ & $2.932 E+02$ & & $2.991 E+02$ & $2.932 E+02$ & $2.961 \mathrm{E}+02$ \\
\hline Molecular Weight & $2.861 E+01$ & $2.86 \div E+01$ & & $2.861 E+01$ & $2.884 E+01$ & $2.873 \mathrm{E}+01$ \\
\hline Pvap (atm) & $2.288 \mathrm{E}-02$ & $2,296 \mathrm{E}-02$ & & $2.296 \mathrm{E}-02$ & 1.125E-03 & $1.204 \mathrm{E}-02$ \\
\hline Psat (etm) & 2.288E-02 & $2.290 \mathrm{E}-02$ & & $3.279 \mathrm{E}-02$ & $2.288 \mathrm{E}-02$ & $2.742 \mathrm{E}-02$ \\
\hline Dow Polnt (C) & $1.992 E+01$ & $1.997 \mathrm{E}+01$ & & $1.997 \mathrm{E}+01$ & $-2.080 E+01$ & $9.985 E+00$ \\
\hline Humldity $(\%)$ & $1.000 E+02$ & $1.002 E+02$ & & $7.000 E+01$ & $4.918 \mathrm{E}+00$ & $4.390 E+01$ \\
\hline$\Delta \mathrm{T}(\mathrm{C})$ & & $4.265 \mathrm{E}-02$ & & $5.985 \mathrm{E}+00$ & & $1.298 \mathrm{E}+01$ \\
\hline
\end{tabular}

